Tohoku Math. J

57 (2005), 65-117

\title{
INFINITE DIMENSIONAL ALGEBRAIC GEOMETRY; ALGEBRAIC STRUCTURES ON $p$-ADIC GROUPS AND THEIR HOMOGENEOUS SPACES
}

\author{
WILLIAM J. HABOUSH
}

(Received June 2, 2003, revised November 12, 2003)

\begin{abstract}
Let $k$ denote the algebraic closure of the finite field, $\mathbb{F}_{p}$, let $\mathcal{O}$ denote the Witt vectors of $k$ and let $K$ denote the fraction field of this ring. In the first part of this paper we construct an algebraic theory of ind-schemes that allows us to represent finite $K$ schemes as infinite dimensional $k$-schemes and we apply this to semisimple groups. In the second part we construct spaces of lattices of fixed discriminant in the vector space $K^{n}$. We determine the structure of these schemes. We devote particular attention to lattices of fixed discriminant in the lattice, $p^{-r} \mathcal{O}^{n}$, computing the Zariski tangent space to a lattice in this scheme and determining the singular points.
\end{abstract}

1. Introduction. In the late 80's and early 90's conjectural variants of the Verlinde formulae and the emergent arithmetic theory of loop groups inspired a great deal of work (See [BLS], [F], [KNR]). The ingredients of that work are by now well-known. They include the moduli of vector bundles of fixed determinant on a pointed Riemann surface, infinite Grassman varieties, spaces of generalized theta functions, weight spaces of certain representations of affine Kac-Moody algebras and the Picard group of a certain generalized Schubert cell in an infinite Grassmannian. As a practitioner of geometry over fields of positive characteristic certain questions naturally occurred to me in response to this work. This program has been generalized to arbitrary fields with notable success by Mathieu [Ma] and Tits [T1]. In Mathieu the emphasis was positive characteristic and Frobenius splitting methods and their application to the characteristic zero case. My attention has been drawn by the possibility of treating the group of points of a split semisimple group in an unramified extension of the complete $p$ adics as a pro-ind-variety over the residue class field. In place of vector bundles, the residue extension and theta functions, one encounters Galois representations, the norm residue symbol and the central extensions studied by Moore [Mo] and Steinberg [St] among others, and Kloosterman and other exponential sums. One finds oneself confronting class field theory and the Langlands program. These are not matters of small importance and so pursuing this analogy is a matter of some mathematical seriousness.

Certain difficulties immediately appear. For one, to be able to define the product structure on the fraction fields of Witt vectors and hence on matrix groups over them, one must pass to function rings closed under taking $p$ 'th roots. These rings have no derivations and so tangent spaces and Lie algebras are non-existent. On the other hand, all integral extensions are separable. In order to define these group scheme like objects, one must construct a theory of

2000 Mathematics Subject Classification. Primary 20G25; Secondary 20G99, 14L15.

Key words and phrases. Group schemes, Witt vectors, lattices, Hilbert class field. 
localized Greenberg functors (see [MJG1], [MJG2]). These notions are canonically imposed on the researcher by the problem. This is the substance of part I of this paper.

Furthermore, I chose to approach these objects in terms of topological algebraic geometry. That is, one considers rings with a linear topology and the space of open primes under the topological Zariski topology. A topological scheme is a local ringed space locally isomorphic to such spaces. Classical formal schemes are subsumed as non-reduced objects. This has the advantage that objects are defined in a fashion that makes them independent of their particular representation as inductive limits.

Spaces of sublattices of vector spaces over certain complete valued fields can be constructed. Remarkably they can be constructed in the category of inductive limits of finite projective schemes. One need not pass to a category of perfect schemes in which functions have infinite $p$ 'th roots, but this comes at a price in at least two different ways. First of all, the action even of the maximal bounded subgroup is not always defined directly, and one must make use of relatively intricate strategies involving Frobenius covers to study orbit structure etc. Secondly, the spaces are constructed using the Hilbert scheme rather than an infinite Grassman variety and so computations can be quite unwieldy. There are alternative ways of describing the schemes of which one takes an inductive limit. The description I have used results in very natural inclusions in the inductive limit. The construction which permits the most natural description of the orbit structure, however, requires a rather intricate system of maps in the limit construction. These conflicting demands have been the peculiar difficulty of this work. Nonetheless, the outlines of a theory do emerge.

These spaces of lattices which are constructed here are of course special to $S L(n, K)$. The homogeneous spaces corresponding to other semisimple groups remain to be constructed. In the last section I give an indication of how I hope to approach the general case. After slightly rephrasing certain well-known results of Bruhat and Tits, I expect to represent these spaces as orbits in the lattice varieties constructed in this paper.

The paper is constructed as follows. Let $p$ be a prime fixed once and throughout and let $k$ be the algebraic closure of $\mathbb{F}_{p}$. Let $\mathcal{O}$ denote the ring of Witt vectors of $k$ and let $K$ be the fraction field of $\mathcal{O}$. Let $F$ be a free $\mathcal{O}$-sub-module of $K^{n}$ of rank $n$. A special $n$-lattice in $K^{n}$ is one which is an image of $F$ under the action of an element of $S L(n, K)$. Then one may view $S L(n, K)$ as operating pointwise on the set of special lattices in $K^{n}$.

The first problem solved in this paper is the extension of the notion of Greenberg functor from schemes of finite type over a discrete valuation ring to schemes of finite type over a complete valued field. This involves the difficulties alluded to above, but it is the only construction I could think of which gives a unique sense to the objects under discussion in this paper. Apart from the necessity of this notion to defining certain objects in this paper it does not find wide use here.

The second problem studied in this paper is the construction of schemes of lattices of various types. The basic construction on which all other constructions are based is the construction of a scheme parametrizing special lattices inside $p^{-r} F$. These schemes are shown 
to be reduced and irreducible. Their tangent spaces are computed and it is shown that their singular loci are of codimension two.

In a planned sequel to this paper I will discuss the action of $S L(n, K)$ on the space of special lattices in some detail. I will consider the Picard group of the lattice scheme and the Mumford groups (see [BLS] for a definition) of certain line bundles. They will be related to the norm residue symbol and the central extensions studied by Moore [Mo], Steinberg [St] and Matsumoto [Mt]. I will also show that these spaces can be modified to produce spaces of Galois representations split by an Abelian extension unramified at $p$.

It is appropriate to mention sources of inspiration and some missed references for much in this paper. The idea of viewing infininite dimensional varieties as topological schemes already appears in some form in Shafarevich [Sh]. The idea of a topological Hopf algebra appears again in Abe and Takeuchi [AT]. In addition, Takeuchi has written of topological Hopf algebras in [MT]. In any case these writers have observed a number of phenomena which motivated this work. I very recently found that Serre has discussed "quasi-algebraic" groups, that is, algebraic objects with a multiplication defined only up to inseparable extension. I would conjecture that his interest was drawn by the same groups that have interested me in this paper. This is to be found in [S2]. I also recently rediscovered the classical work of Barsotti whose papers written in the 60's ([IB1], [IB2], [IB3]) contain a related theory. In any case the necessity of working with topological schemes is, I believe, clear to many, but the necessity of constructing foundations has been a serious deterrent. It is quite clear that this work must be done if one is to bring techniques from conformal field theory and the theory of moduli of principle bundles to class field theory, the Langlands program, the study of Galois representations and the theory of automorphic forms and it is equally clear that this is the only way to approach central issues in these areas.

Finally I would like to thank Tadao Oda for a number of very helpful suggestions. He read an earlier version of this paper and made a number of valuable observations.

\section{Topological algebraic geometry.}

2.1. Topological rings; formal spectra: The field, $k$, is fixed throughout. We assume that it is of characteristic, $p$. If $p>0$ and $r \geq 0$ and if $X$ is a $k$-scheme, $X^{(r)}$ denotes the $r^{\prime}$ th Frobenius cover, $X^{(r)} \rightarrow X$.

Definition 1. Let $V$ be a vector space over $k$. A linear topology on $V$ is a topology on $V$ for which there exists a basis of neighborhoods of 0 consisting of vector subspaces of $V$. If $R$ is a $k$-algebra and $M$ is an $R$-module, a linear topology on $M$ is one for which there is a basis of neighborhoods of 0 consisting of $R$-submodules of $M$. This applies to $R$ itself as well. A $k$-algebra with a linear topology will be called a topological $k$-algebra or sometimes just a topological ring. If $R$ is a topological $k$-algebra, an $R$-module endowed with a linear topology will be called a topological $R$-module if for each open submodule, $N$, the ideal, $(N: M)=\{x \in R: x M \subseteq N\}$ is open. 
If $R$ is a topological ring and $I$ is a closed ideal, then $R / I$ is canonically endowed with a linear topology for which the surjection is a topological quotient morphism. The same is so for modules.

For any proper open ideal $I$, the topology on $R / I$ is the discrete topology. There is always a canonical map, $R \rightarrow \lim _{I \text { open }} R / I$. Then $R$ is separated if this map is injective; complete if it is surjective. The same is true for modules or indeed for topological vector spaces. The limit, $\lim _{I \text { open }} R / I$, is the completion of $R$. The same discussion and terminology apply to modules and vector spaces.

If $R$ is a topological ring, its topological nil-radical is the set of elements, $x$, such that the sequence, $x^{n}$, converges to 0 . It is an ideal and $R$ is called topologically reduced if its topological nil-radical is (0). If $R$ is a topological ring and $S$ is a multiplicatively closed set, then the localization, $R_{S}$, is canonically a topological ring. Just declare $J \subset R_{S}$ to be open if its inverse image in $R$ is. The complete localization of $R$ at $S$ is the completion of $R_{S}$. We shall write it $R_{\langle S\rangle}$. Notice that the kernel of the natural map from $R$ to $R_{\langle S\rangle}$ is the ideal $\mathfrak{n}_{S}=\{r: 0 \in \overline{r S}\}$. Here, $r S$ is the set, $\{r s: s \in S\}$, and the bar denotes closure. It should be noted that $R_{\langle S\rangle}=(0)$ when $\bar{S} \cap \mathfrak{n} \neq \emptyset$.

With these notions, we may construct a certain local ringed space, $\operatorname{Spf}(R)$, for each topological ring, $R$. This is the formal spectrum as in [EGA] or as it is presented in [Ha] but we shall write it out for good measure. Both a $k$-algebra and its completion yield the same result and so we will henceforth assume that $R$ is complete and separated. The underlying topological space of $\operatorname{Spf}(R)$ is the set of open prime ideals. The closed subsets of $\operatorname{Spf}(R)$ are sets of the form $\mathcal{V}(I)=\{\mathfrak{p}: \mathfrak{p} \in \operatorname{Spf}(R), \mathfrak{p} \supseteq I\}$ for $I$ some closed ideal of $R$. In particular, the sets, $D(f)=\{\mathfrak{p}: f \notin \mathfrak{p}\}$, are a subbase for the topology of $\operatorname{Spf}(R)$. One may define a sheaf by giving it on a subbase. Set $\tilde{\mathcal{O}}_{R}(D(f))$ equal to $R_{\langle f\rangle}$ where this denotes the complete localization at the set of powers of $f$. It must be shown that this depends only on the open set, $D(f)$, and not on $f$. The complete localization is the projective limit of the rings, $R_{f} / I R_{f}$ over all open $I$ such that $f \notin I$. Suppose that $D(f)=D(g)$. Then for all open $I$ such that $f \notin I, R_{f} / I R_{f}=R_{g} / I R_{g}$, and so the inverse limit of these rings which is the complete localization, depends only on the set of open primes, $D(f)$. Let $\mathcal{O}_{R}$ be the sheaf associated to this presheaf. Notice that this is a sheaf of topological rings. The pair consisting of the topological space just constructed together with the sheaf given here is the local ringed space, $\operatorname{Spf}(R)$. Note that when the topology on $R$ is discrete, $\operatorname{Spf}(R)$ is just $\operatorname{Spec}(R)$.

Notice that if $I$ is any ideal then since every open ideal is closed, any open prime containing $I$ also contains $\bar{I}$ and so $\mathcal{V}(I)=\mathcal{V}(\bar{I})$. Hence $\bigcap_{i} \mathcal{V}\left(I_{i}\right)=\mathcal{V}\left(\overline{\sum_{i} I_{i}}\right)$. This means that $\operatorname{Spf}(R)$ need not be quasi-compact since it may be that $\overline{\sum_{i} I_{i}}=R$ even when $\sum_{i} I_{i} \neq R$. It is also worth observing that $\operatorname{Spf}(R)=\underline{\lim }_{I \text { open }} \operatorname{Spec}(R / I)$ where this last limit is in the category of ringed spaces and is hence an inductive limit on spaces and a projective limit on sheaves.

DEFINITION 2. Let $R$ be a complete separated $k$-algebra with a linear topology. Then the formal spectrum of $R$, written $\operatorname{Spf}(R)$, is the set of open primes in $R$ endowed with the 
topology and the sheaf of topological rings described above. A topological scheme is a local ringed space $\left(X, \mathcal{O}_{X}\right)$ such that $X$ admits a covering by open sets, $U$, such that $\left(U, \mathcal{O}_{X} \mid U\right)$ is the formal spectrum of some topological ring. A morphism of topological schemes, $\tilde{f}=$ $\left(f, f^{\circ}\right)$, is a morphism of ringed spaces, $\tilde{f}: X \rightarrow Y$ such that for each open set $U \subset Y$ the morphism, $f_{U}^{\circ}: \mathcal{O}_{Y}(U) \rightarrow \mathcal{O}_{X}\left(f^{-1}(U)\right)$ is continuous.

Since $\operatorname{Spf}(R)$ is just $\operatorname{Spec}(R)$ when the topology on $R$ is discrete, the category of $k$ schemes is a full subcategory of the category of topological schemes over $k$. The formal dimension of a formal scheme will be one less than the maximal length of a chain of open primes. Thus the formal dimension of a scheme with the discrete topology on its sheaf of rings is just its dimension as a scheme, while the formal dimension of the formal spectrum of a complete local ring is zero.

If $R$ and $S$ are topological $k$-algebras, then $R \otimes_{k} S$ is as well (with open ideals $I \otimes_{k}$ $S+R \otimes_{k} J$ for $I$ and $J$ open). Let $R \hat{\otimes}_{k} S$ denote the complete tensor product. It is readily seen to be the coproduct of $R$ and $S$ in the category of topological $k$-algebras. When $R \rightarrow S$ is a continuous morphism of topological $k$-algebras, we shall call $S$ a topological $R$-algebra. If $S$ and $T$ are topological $R$-algebras, then one may construct the relative cofibre coproduct, $S \hat{\otimes}_{R} T$. Since it is a relative coproduct, it is clear that $\operatorname{Spf}\left(S \hat{\otimes}_{R} T\right)$ is the relative fiber product, $Y \times{ }_{X} Z$, for $X=\operatorname{Spf}(R), Y=\operatorname{Spf}(S)$ and $Z=\operatorname{Spf}(T)$.

The existence of relative fiber products of affine topological schemes over an affine base, that is, of formal spectra over a formal spectrum, allows us to apply any of the standard arguments for the existence of relative fiber products of schemes to deduce that relative fibre products of topological schemes exist. The seven steps in the proof of 3.3 on pages 87 and 88 of [Ha], for example, apply without a word of change and so the following holds:

Proposition 1. Let $f: X \rightarrow S$ and $g: Y \rightarrow S$ be two topological morphisms of topological $k$-schemes. Then, the relative fiber product, $X \times_{S} Y$ exists in the category of topological schemes.

One may now speak of separated topological morphisms. Namely, $f: X \rightarrow Y$ is separated if and only if the diagonal $\Delta: X \rightarrow X \times_{Y} X$ is a closed embedding. Most of the standard notions of algebraic geometry generalize to the category of topological schemes. Except when an alteration or a comment is necessary, they will be used as needed.

A discrete subscheme of $X$ will always mean a closed topological subscheme, $Y \subseteq X$ such that the topology on $\mathcal{O}_{Y}(U)$ is the discrete topology for all open subsets, $U \subseteq Y$.

Definition 3. The topological scheme $X$ will be called pro-Noetherian if it can be written as an inductive limit, $X=\lim _{i \in I} Y_{i}$, of closed discrete Noetherian subschemes, $Y_{i}$.

Notice that it is possible to define topological group schemes over $S$. Namely $f: G \rightarrow S$ together with the morphisms, $\mu: G \times{ }_{S} G \rightarrow G, s: G \rightarrow G$ and $e: S \rightarrow G$, is a topological group scheme if the data satisfy the usual axioms. One must always, however, require that the set of $G$-points, $G(Y)=\operatorname{Hom}_{S}(Y, G)$ be the set of morphisms in the topological category. With that understanding, a topological group scheme is just a group in the category of 
topological schemes. Then, as is generally true in a category with products, it may also be characterized as a topological scheme whose functor of points is equipped with a structure as a functor to the category of groups.

2.2. Perfect algebraic geometry: Many of the algebraic objects considered in this paper are not just topological, they are also spectra of perfect rings. Assume that $k$ is a perfect field of characteristic $p>0$. Let $R$ be a $k$-algebra. Then $R$ will be called perfect if it is reduced and the Frobenius morphism, $F: R \rightarrow R, F(a)=a^{p}$, is surjective. An ideal, $I$, in a perfect ring will be called perfect if it is radical and equal to its own image under the Frobenius morphism. If $R$ is perfect and $\delta$ is any derivation of $R$ in an $R$-module, $M$, then $\delta=0$ because every element of $R$ is a $p$ 'th power.

If $R$ is a commutative $k$-algebra, form an inductive system by setting $R_{n}=R, n \in$ $\mathbb{Z}^{+}$and taking the map from $R_{n}$ to $R_{m}$ to be $F^{m-n}$ for $m \geq n$. This is a direct system of commutative rings whose limit we write $R_{\infty}$. It is the perfect closure of $R$. It can be thought of as the ring of equivalence classes of symbols, $[a]_{n}, n \geq 0$, with the operations, $[a]_{n}+[b]_{n}=$ $[a+b]_{n},[a]_{n}[b]_{n}=[a b]_{n}$ and subject to the equivalence relation, $\left[a^{p^{r}}\right]_{n+r}=[a]_{n}$. Notice that the perfect closure is always reduced.

Write $k\left[x_{i} ; i \in I\right]_{\infty}$ for the perfect closure of the ring of polynomials in the variables $\left\{x_{i}\right\}_{i \in I}$. It consists of sums of monomials in the $x_{i}$ with exponents in the positive elements of the ring $\mathbb{Z}[1 / p]$, the localization of $\mathbb{Z}$ at $p$. This ring of polynomials may be viewed as the corresponding monoid algebra. We shall call this ring the ring of perfect polynomials in the $x_{i}$.

It is of some interest to note what the homomorphisms from $k[x]_{\infty}$, the perfect polynomials in one variable, to the $k$-algebra, $A$, are. If $A$ has $p$-nilpotents, they correspond to the sequences, $\left\{a_{i}\right\}_{i \in \mathbb{Z}}$ such that $a_{i}=a_{i+1}^{p}$. Under coordinate wise multiplication and addition these sequences form a ring. Notice that it is always reduced. If $A$ has no $p$-nilpotents, they correspond to elements with arbitrary $p^{n}$ 'th roots, that is, to the maximal perfect subring. We will write $A_{0}$ for this ring of sequences. For convenience, in characteristic zero, the two symbols, $A_{\infty}$ and $A_{0}$, will both be taken to be $A$ itself. We shall refer to $A_{0}$ as the the ring of perfect values in $A$. We shall call the map which sends the sequence, $\left\{a_{i}\right\}_{i \in \mathbb{Z}}$, to $a_{0}$ simply the canonical map.

We begin with an example of a perfect scheme. Since the topology on its functions is the discrete topology it is in fact a perfect group scheme. It will appear frequently in what follows. For example if $\mathcal{O}$ is a complete discrete valuation ring of characteristic 0 with residue class field, $k$, and fraction field, $K$, the maximal $k$-torus of a group of the form $G(K)$ where $G$ is linear algebraic is of this type. We include it to point out some of the problems involved with perfect schemes and also to show that they can nonetheless be rather manageable.

DEFINITION 4. Let $\Gamma$ be a $\mathbb{Z}[1 / p]$-module. Then the perfect multiplicative group associated to $\Gamma$ is the group of multiplicative type associated to $\Gamma$ over $k$ in the sense of [SGAD, II]. That is, it is the spectrum of the group algebra of $\Gamma$ with co-multiplication, $\mu(\gamma)=\gamma \otimes \gamma$, co-unit $e(\gamma)=1$ and antipode $s(\gamma)=\gamma^{-1}$. 
Thus the perfect multiplicative group associated to $\Gamma$ is an unremarkable group scheme of multiplicative type in the sense of [SGAD, II] except that when it is not finite it is not Noetherian and moreover, since its coordinate ring is perfect, it has Lie algebra (0). It is possible to compute its ring of invariant differential operators. We shall indicate how to do it for $\Gamma$ of the form $\mathbb{Z}[1 / p]^{\oplus n}$. It is important to observe that an affine action of the perfect multiplicative group associated to $\Gamma$ is just a $\Gamma$-graded ring. In what follows affine actions of the group associated to $\mathbb{Z}[1 / p]$ will play a crucial role.

Observe that if $\left(\begin{array}{l}n \\ r\end{array}\right)$ is the binomial coefficient, it satisfies the identity $\left(\begin{array}{l}n \\ r\end{array}\right) \equiv\left(\begin{array}{c}p n \\ p r\end{array}\right) \bmod p$. By abuse of language view binomial coefficients as functions with values in the field with $p$ elements. For $r$ and $v \in \mathbb{Z}[1 / p]$ with $r \geq 0$, define the value of $\left(\begin{array}{l}v \\ r\end{array}\right)$ as $\left(\begin{array}{l}p^{s} v \\ p^{s} r\end{array}\right)$ for $s$ large enough to clear denominators. By the identity observed above, this is independent of $s$ so long as $s$ clears denominators. Write the elements of $\mathbb{Z}[1 / p]^{\oplus n}$ as $n$-tuples $\left(v_{1}, \ldots, v_{n}\right)$. Then for any $n$-tuple, $\boldsymbol{v}$, and non-negative $n$-tuple, $\mathbf{r}$, let $\left(\begin{array}{c}\boldsymbol{v} \\ \mathbf{r}\end{array}\right)=\prod_{i=1}^{n}\left(\begin{array}{c}v_{i} \\ r_{i}\end{array}\right)$.

Now for any $n$-tuple, $v \in \mathbb{Z}[1 / p]^{\oplus n}=\Gamma$ write $t^{v}$ for that element viewed as an element of the group algebra, $k[\Gamma]$. Define a symbol, $\left(\begin{array}{c}\mathbf{H} \\ \mathbf{r}\end{array}\right)$ for $\mathbf{r}$ a non-negative element of $\Gamma$ as follows. The symbol, $\left(\begin{array}{c}\mathbf{H} \\ \mathbf{r}\end{array}\right)$, is an element of the linear dual of $k[\Gamma]$ defined by the linear pairing, $\left\langle\left(\begin{array}{l}\mathbf{H} \\ \mathbf{r}\end{array}\right), t^{\boldsymbol{v}}\right\rangle=\left(\begin{array}{l}\boldsymbol{v} \\ \mathbf{r}\end{array}\right)$. Then $\left(\begin{array}{l}\mathbf{H} \\ \mathbf{r}\end{array}\right)$ is a distribution on the perfect multiplicative group associated to $\Gamma$ and its convolution action is given by $\left(\begin{array}{c}\mathbf{H} \\ \mathbf{r}\end{array}\right) * t^{\boldsymbol{v}}=\left(\begin{array}{l}\boldsymbol{v} \\ \mathbf{r}\end{array}\right) t^{\boldsymbol{v}}$. The ring of invariant differential operators on $\operatorname{Spec}(k[\Gamma])$ is a completion of $D$, the linear span of these symbols. Namely, let $\Gamma_{0}$ be the $\mathbb{Z}$-lattice $\mathbb{Z}^{\oplus n} \subseteq \Gamma$ and, for each $q \in \mathbb{Z}$, let $I_{q}$ be the ideal of elements in $D$ which vanish on $p^{q} \Gamma_{0}$. Regard these ideals as a basis of neighborhoods of (0) and complete $D$ accordingly and call the ring obtained $\mathcal{D}_{\Gamma}$. To see that this is the appropriate ring, just note that the $k[\Gamma]$ is an inductive limit and that $\mathcal{D}$, the completion just described is just the dual projective limit.

It is of some interest to note that the notion of a perfect closure is geometric. Let $X$ be any scheme of finite type over the field, $k$. Now for any $k$-algebra, $R$, let $R^{1 / p^{n}}$ the $R$-algebra whose underlying set is $R$ but which is an $R$-algebra by means of the $p^{n}$ 'th power morphism. Define a sheaf, $\mathcal{O}_{X}^{1 / p^{n}}$, by the equation, $\mathcal{O}_{X}^{1 / p^{n}}(U)=\mathcal{O}_{X}(U)^{1 / p^{n}}$. Then the space, $X$, with the sheaf $\mathcal{O}_{X}^{1 / p^{n}}$ is a scheme and a purely inseparable cover of $X$. Set $\mathcal{O}_{X}^{p^{-\infty}}=\lim _{n} \mathcal{O}_{X}^{1 / p^{n}}$. The space, $X$, with the sheaf, $\mathcal{O}_{X}^{1 / p^{n}}$, restricts to a true scheme on closed sets defined by open ideals. It is hence natural to consider the space with the sheaf associated to the presheaf of completions on open sets. It is a topological scheme with the same space. Since the corresponding sheaf is the one most natural to our discussion, we write this sheaf associated to the completions as $\hat{\mathcal{O}}_{X}^{1 / p^{-\infty}}$ but more briefly as $\mathcal{O}_{X}^{\infty}$.

Definition 5. Let $X_{\infty}$ denote the space, $X$, together with the sheaf $\hat{\mathcal{O}}_{X}^{p^{-\infty}}$. It will be referred to as the complete perfect cover of $X$. Let $X_{\infty}^{0}$ denote the same space with the sheaf $\mathcal{O}_{X}^{p^{-\infty}}$. This will be called the incomplete perfect cover of $X$. 
This distinction between the complete and incomplete perfect covers is crucial. The infinite polynomials defining multiplication on the fraction field of the Witt vectors, for example, are in the complete perfect cover but not the incomplete perfect cover. On the other hand, the incomplete perfect cover will play an important role in the definition of the localized Greenberg functor.

2.3. Some important topological schemes: In this section, we shall consider two distinct types of infinite dimensional affine spaces which will play a special role in what follows. Write $R\left[x_{i}\right]_{i \in \mathbb{Z}}$ to denote the ring of polynomials over $R$ in a set of indeterminates indexed by the integers. We define two sequences of ideals, $J_{v}$ and $L_{v}, v \geq 0$ each of which is a basis of neighborhoods of 0 . First $J_{v}$ is the ideal in $R\left[x_{i}\right]_{i \in \mathbb{Z}}$ generated by $\left\{x_{i}: i<v\right\}$. The ideal $L_{v}$ is the ideal generated by the set, $\left\{x_{i}: i<-v\right\} \cup\left\{x_{i}: i>v\right\}$. We assume that $R$ is discrete.

Definition 6. Let $R$ be a discrete ring. Then $R^{+}\left\langle X_{\mathbb{Z}}\right\rangle$ will denote the completion of the ring, $R\left[x_{i}\right]_{i \in \mathbb{Z}}$ in the topology in which $\left\{J_{v}\right\}_{v \in \mathbb{Z}}$ is a basis of neighborhoods of 0 . We shall write $R^{L}\left\langle X_{\mathbb{Z}}\right\rangle$ for the completion of $R\left[x_{i}\right]_{i \in \mathbb{Z}}$ in the topology in which the ideals, $\left\{L_{v}\right\}_{v \geq 0}$, are a basis of neighborhoods of 0 .

We wish to give very explicit descriptions of these rings. First, $R^{+}\left\langle X_{\mathbb{Z}}\right\rangle$ may be thought of as a ring of infinite polynomials, $u$, described as follows. The element $u$ is an infinite linear combination of monomials subject to the requirement that for any integer, $n$, the set of monomials exclusively in the indeterminates, $x_{i}$, for $i>n$ and having a non-zero coefficient in $u$ is finite in number.

The ring $R^{L}\left\langle X_{\mathbb{Z}}\right\rangle$ is also a ring of infinite polynomials, $u$, but the condition is different. In this case $u$ is an infinite linear combination of monomials subject to the requirement that for any non-negative integer, $n$, the set of monomials exclusively in the indeterminates, $\left\{x_{i}\right.$ : $-n<i<n\}$, and having non-zero coefficients in $u$ is finite in number.

One may easily verify that in either of these rings of infinite polynomials the multiplication is perfectly well defined in the most trivial sense. Moreover the elements of these rings define functions on certain easily understood spaces. For any discrete $R$-algebra, $B$, let $\mathbb{A}_{R}^{+}(B)$ denote the set of sequences $\left\{b_{i}\right\}, b_{i} \in B$ for $i$ in $\mathbb{Z}$ such that for some $n, b_{i}=0$ for all $i<n$ and let $\mathbb{A}_{R}^{L}(B)$ denote the set of sequences $\left\{b_{i}\right\}$ such that there is an $n>0$ such that $b_{i}=0$ for all $i$ such that $|i|>n$. Then, examining the elements of $R^{+}\left\langle X_{\mathbb{Z}}\right\rangle$ and $R^{L}\left\langle X_{\mathbb{Z}}\right\rangle$, one easily sees that by substituting the $b_{i}$ for the $x_{i}$, the elements of $\mathbb{A}_{R}^{+}(B)$ and $\mathbb{A}_{R}^{L}(B)$ define homomorphisms from $R^{+}\left\langle X_{\mathbb{Z}}\right\rangle$ and $R^{L}\left\langle X_{\mathbb{Z}}\right\rangle$ to $B$, respectively. Finally bear in mind that, for $B$ discrete, $\operatorname{Spf}(B)=\operatorname{Spec}(B)$.

DEFINITION 7. We shall refer to $R^{+}\left\langle X_{\mathbb{Z}}\right\rangle$ as the ring of infinite Laurent coefficients over $R$ and to $R^{L}\left\langle X_{\mathbb{Z}}\right\rangle$ as the ring of finite Laurent coefficients over $R$.

Proposition 2. Let $B$ be a discrete R-algebra. Then,

(1) The set of continuous homomorphisms from $R^{+}\left\langle X_{\mathbb{Z}}\right\rangle$ to $B$ is in bijective correspondence with $\mathbb{A}_{R}^{+}(B)$. 
(2) The set of continuous homomorphisms from $R^{L}\left\langle X_{\mathbb{Z}}\right\rangle$ to $B$ is in bijective correspondence with $\mathbb{A}_{R}^{L}(B)$.

That is $\mathbb{A}_{R}^{+}(B)$ is the set of $\operatorname{Spf}(B)$-valued points of $\operatorname{Spf}\left(R^{+}\left\langle X_{\mathbb{Z}}\right\rangle\right)$ and $\mathbb{A}_{R}^{L}(B)$ is the set of $\operatorname{Spf}(B)$-valued points of $\operatorname{Spf}\left(R^{L}\left\langle X_{\mathbb{Z}}\right\rangle\right)$.

Proof. Let $\phi$, respectively $\psi$, denote a continuous homomorphism from $R^{+}\left\langle X_{\mathbb{Z}}\right\rangle$, respectively $R^{L}\left\langle X_{\mathbb{Z}}\right\rangle$, to $B$. Thus $\phi$ and $\psi$ must each vanish on an open ideal. Hence $\phi$ must vanish on an ideal of the form, $\hat{I}_{\nu}$, generated by the set of $x_{n}$ for $n<v$, while $\psi$ must vanish on some $\hat{J}_{v}, v \geq 0$ generated by the set of $x_{n}$ such that $n<-v$ or $n>v$. But $R^{+}\left\langle X_{\mathbb{Z}}\right\rangle / \hat{I}_{v}=R\left[x_{v}, x_{v+1}, \ldots\right]$ and $R^{L}\left\langle X_{\mathbb{Z}}\right\rangle / \hat{J}_{v}=R\left[x_{-v}, x_{-v+1}, \ldots, x_{v}\right]$. Consequently, $\phi$ is determined by the values, $b_{i}$, it assigns to the $x_{i}, i \geq v$ which may be chosen arbitrarily and $\psi$ is determined by the values $b_{i}$ it assigns to the finite set, $x_{-v}, x_{-v+1}, \ldots, x_{v}$ which may be chosen arbitrarily.

Because of this proposition, we write $\mathbb{A}_{R}^{+}=\operatorname{Spf}\left(R^{+}\left\langle X_{\mathbb{Z}}\right\rangle\right)$ and $\mathbb{A}_{R}^{L}=\operatorname{Spf}\left(R^{L}\left\langle X_{\mathbb{Z}}\right\rangle\right)$. This is also an appropriate time to introduce some conventions. When $I$ is an index set for a set $y_{i}$ of indeterminates, we shall write $Y_{I}$ for the set and, when the meaning is clear, write such expressions as $R\left[Y_{I}\right]$. Further, when there is no ambiguity concerning the index set, we may write merely $Y$ and $R[Y]$ for $Y_{I}$ and $R\left[Y_{I}\right]$.

It is of some interest to consider the perfect completion. We will give an element in the perfect completion of $k^{+}\left\langle X_{\mathbb{Z}}\right\rangle$ which is not in its perfect closure. Just consider $\sum_{i \leq 0} x_{i}^{p^{i}}$. As the elements of the ordinary (algebraic) perfect closure would have $p$-denominators in their exponents bounded from below, this element is not in the perfect closure. For all of these constructions, we shall indicate the corresponding perfect completion by placing the subscript, $\infty$, on the ring of coefficients. The perfect completion will be indicated by the addition of the subscript, $\infty$. Thus we will write $k_{\infty}^{+}\left\langle X_{\mathbb{Z}}\right\rangle, k_{\infty}^{L}\left\langle X_{\mathbb{Z}}\right\rangle, X_{\infty}$ and so on. The formal spectrum of the perfect completion of a topological algebra is the complete perfect cover of the formal spectrum of that algebra.

2.4. The projective space associated to a topological vector space: If $V$ is a vector space over $k$ with a linear topology, the symmetric algebra, $S_{k}(V)$, carries a natural topology determined by that on $V$. Namely, an ideal in $S_{k}(V)$ is open if and only if it contains an open subspace of $V$. We shall call this the topology on $S_{k}(V)$ induced by that on $V$. This topology, in turn, induces a topology on each of the vector spaces $S_{k}^{q}(V)$. A vector subspace, $M \subseteq S_{k}^{q}(V)$ is open if and only if $M$ contains a set of the form $I \cap S_{k}^{q}(V)$ for some open ideal, $I$. Clearly, the closure of $S_{k}^{q}(V)$ in the completion of $S_{k}(V)$ is its closure in this induced topology.

Definition 8. Let $V$ be a $k$-vector space with a linear topology. The complete symmetric algebra on $V$, written $\hat{S}_{k}(V)$, will signify the completion of $S_{k}(V)$ in the topology on $S_{k}(V)$ induced by that on $V$. The complete $q$ 'th symmetric power of $V$, written $\hat{S}_{k}^{q}(V)$, will refer to the closure of the image of $S_{k}^{q}(V)$ in $\hat{S}_{k}(V)$. The complete graded symmetric algebra 
on $V$ will mean the sub-algebra, $\bigsqcup_{q \geq 0} \hat{S}_{k}^{q}(V)$, of $\hat{S}_{k}(V)$. We shall write it $\hat{S}_{k}^{\sharp}(V)$. The formal spectrum of $\hat{S}_{k}(V)$ will be written $\breve{V}$. It will be called the associated affine space of $V$.

Notice that the set of $k$-points of $\check{V}$ is exactly the continuous dual of $V$ and that the $\operatorname{Spf}(R)$-valued points are just the continuous homomorphisms from $V$ to $R$. This is simple enough, but the analogue of a projective space is somewhat more problematic.

DEFINITION 9. A topological graded algebra is a graded algebra, $S=\bigsqcup_{v} S_{v}$ such that each of the homogeneous parts, $S_{r}$, is endowed with a linear topology and so that multiplication, $S_{r} \times S_{q} \rightarrow S_{r+q}$ is jointly continuous for all $r, q$. We shall call $S$ separated if each of the $S_{r}$ is, and complete if each of the $S_{r}$ is. An ideal is graded if it is the direct sum of its intersections with each of the homogeneous summands $S_{q}$. The irrelevant ideal is the sum, $\bigsqcup_{q>0} S^{q}$. An ideal is open if and only if its intersection with $S_{q}$ is for each $q$.

This last condition specifies a topology on $S$, namely the coproduct topology. Notice that each open ideal contains an open subset of $S_{1}$ and hence the ideal generated by it.

We construct a topological space associated to the topological graded algebra, $S$. Its points are the graded open primes, $\mathfrak{P}$, in $S$ which do not contain the irrelevant ideal. If $I$ is any graded ideal, let $\mathcal{V}^{+}(I)$ denote the set of non-irrelevant open graded primes containing $I$. Then $\mathcal{V}^{+}(I \cap J)=\mathcal{V}^{+}(I) \cup \mathcal{V}^{+}(J)$ and $\mathcal{V}^{+}\left(\sum_{i \in \mathcal{I}} I_{i}\right)=\bigcap_{i \in \mathcal{I}} \mathcal{V}^{+}\left(I_{i}\right)$ and so the sets, $\mathcal{V}^{+}(I)$, may be taken as the closed sets in a topology on the set of graded primes. Write $D^{+}(I)$ for the complement of $\mathcal{V}^{+}(I)$. Also write $\mathcal{V}^{+}(f)$ and $D^{+}(f)$ for $\mathcal{V}^{+}(f S)$ and its complement, respectively. We shall write $\operatorname{FProj}(S)$ for this topological space. Its open sets are the sets, $D^{+}(I)$.

There are at least two different ways of constructing a sheaf on $\operatorname{FProj}(S)$. The first is to observe that, as a topological space, $\operatorname{FProj}(S)$ is the direct limit of the closed subspaces $\operatorname{Proj}(S / I)$ as $I$ ranges over the partially ordered set of open ideals. Corresponding to an inclusion of open ideals, $I \subset J$, there is a closed embedding of schemes, $\operatorname{Proj}(S / J) \subset$ $\operatorname{Proj}(S / I) \subset \operatorname{FProj}(S)$. Hence one may consider the projective limit of the structure sheaves of the schemes $\operatorname{Proj}(S / I)$. This projective limit is the structure sheaf of FProj $(S)$.

Alternatively, let $f$ be a homogeneous element of $S$. Then the localization, $S_{f}$ is a graded topological algebra. In particular, the set of elements of degree 0 in $S_{f}$ is an algebra with a linear topology. Denote it $S_{f}^{0}$. Then $\operatorname{Spf}\left(S_{f}^{0}\right)$ is a topological scheme and its underlying space is evidently equal to $D^{+}(f)$. Then the structure sheaf on $\operatorname{FProj}(S)$ restricts to a sheaf isomorphic to the structure sheaf of $\operatorname{Spf}\left(S_{f}^{0}\right)$ on $D^{+}(f)$. We leave to the reader the problem of showing that this characterization gives the same sheaf as that of the previous paragraph.

DEFINITION 10. Let $S$ be a topological graded algebra. Then, the formal projective scheme associated to $S$, written $\operatorname{Froj}(S)$ is the local ringed space whose underlying topological space is the set of open homogeneous primes endowed with the graded Zariski topology. Its structure sheaf is the sheaf $\mathcal{O}_{\mathrm{FProj}(S)}$ whose restriction to $D^{+}(f)$ is the structure sheaf of $\operatorname{Spf}\left(S_{f}^{0}\right)$ for each homogeneous $f$. 
Finally, we construct the formal projective scheme, $\mathrm{FP}(\check{V})$, whenever $V$ is a topological vector space with associated affine space, $\check{V}$. Then $\mathrm{FP}(\check{V})$ is just $\operatorname{FProj}\left(\hat{S}_{k}^{\sharp}(V)\right)$.

2.5. The Witt fractions: For $k$ a perfect field of characteristic $p>0$ let $\mathfrak{W}(k)=W_{k}$ denote the ring of Witt vectors of $k$. By the Witt fractions of the field, $k$, we shall mean the fraction field of its ring of Witt vectors. Henceforth we shall always write it $K$. We shall construct this field as the $k$-points of a topological scheme. A particular difficulty enters into their construction. As a scheme of points with no algebraic structures, or even as an additive group, the construction presents no problems. To construct a polynomial multiplication, however, we must pass to the complete perfect closure. To fix notation, for any $\xi \in k$ let $\tilde{\xi} \in W$ denote its multiplicative representative. For a $k$-polynomial, $f$, let $\tilde{f}$ denote the function whose value on $x$ is the multiplicative representative of $f(x)$. The infinite vector, $\left(\xi_{0}, \ldots, \xi_{i}, \ldots\right)$ will denote the vector corresponding to the element $\sum_{i \geq 0} \tilde{\xi}_{i}^{p^{-i}} p^{i}$. Let $\Phi_{i}$ and $\Psi_{i}$ be the polynomials defined by the equations,

$$
\sum_{i \geq 0} \tilde{\xi}_{i}^{p^{-i}} p^{i}+\sum_{i \geq 0} \tilde{\eta}_{i}^{p^{-i}} p^{i}=\sum_{i \geq 0} \tilde{\Phi}_{i}\left(\xi_{0}, \ldots, \xi_{i} ; \eta_{0}, \ldots, \eta_{i}\right)^{p^{-i}} p^{i}
$$

and

$$
\left(\sum_{i \geq 0} \tilde{\xi}_{i}^{p^{-i}} p^{i}\right)\left(\sum_{i \geq 0} \tilde{\eta}_{i}^{p^{-i}} p^{i}\right)=\sum_{i \geq 0} \tilde{\Psi}_{i}\left(\xi_{0}, \ldots, \xi_{i} ; \eta_{0}, \ldots, \eta_{i}\right)^{p^{-i}} p^{i} .
$$

Having defined these polynomials in this fashion, we observe the following restriction formulae which result from restricting the $\Phi_{i}$ to $p^{r} W \times p^{r} W$ and the $\Psi_{i}$ to $p^{r} W \times p^{s} W$. The two formulae are just the polynomial equations resulting from the statements, $p^{r} a+p^{r} b=$ $p^{r}(a+b)$ and $p^{r} u \cdot p^{s} v=p^{(r+s)} u v$. To deduce these formulae, first restrict then note that since the coefficients of $\Phi_{i}$ and $\Psi_{j}$ are in the prime field they satisfy the functional equation $f\left(x_{1}, \ldots, x_{m}\right)^{p^{s}}=f\left(x_{1}^{p^{s}}, \ldots, x_{m}^{p^{s}}\right)$. They are:

$$
\begin{gathered}
\Phi_{i}\left(0, \ldots, 0, x_{r}, \ldots, x_{i} ; 0, \ldots, 0, y_{r}, \ldots, y_{i}\right)=\Phi_{i-r}\left(x_{r}, \ldots, x_{i} ; y_{r}, \ldots, y_{i}\right) \\
\Psi_{i}\left(0, \ldots, 0, x_{r}, \ldots, x_{i} ; 0, \ldots, 0, y_{s}, \ldots, y_{i}\right) \\
=\Psi_{i-r-s}\left(x_{r}^{p^{s}}, \ldots, x_{i-s}^{p^{s}} ; y_{s}^{p^{r}}, \ldots, y_{i-r}^{p^{r}}\right) .
\end{gathered}
$$

In these equations, it is understood that the polynomials, $\Phi_{i}$ and $\Psi_{i}$, do not exist when $i<0$. Further, they must be regarded as polynomials over the prime field of characteristic, $p>0$, even though the polynomials themselves are defined over $\mathbb{Z}$, because we must use the functional equations noted above.

We now consider the $x_{i}, i \in \mathbb{Z}$ and the $y_{i}, i \in \mathbb{Z}$ to be elements in the ring of perfect polynomials in those variables. For non-positive integers, $r, s$, define polynomials, $\Phi_{i}^{(r)}$ and $\Psi_{i}^{r, s}$ by the equations:

$$
\begin{aligned}
\Phi_{i}^{(r)}\left(x_{r}, \ldots, x_{i} ; y_{r}, \ldots, y_{i}\right) & =\Phi_{i-r}\left(x_{r}, \ldots, x_{i} ; y_{r}, \ldots, y_{i}\right) \\
\Psi_{i}^{r, s}\left(x_{r}, \ldots, x_{i-s} ; y_{s}, \ldots, y_{i-r}\right) & =\Psi_{i-r-s}\left(x_{r}^{p^{s}}, \ldots, x_{i-s}^{p^{s}} ; y_{s}^{p^{r}}, \ldots, y_{i-r}^{p^{r}}\right) .
\end{aligned}
$$


Then by the restriction formulae, 2.3 and 2.4, for integers $q, m$ such that $q>r$ and $m>s, \Phi_{i}^{(r)} \mid p^{q} W \times p^{q} W=\Phi_{i}^{(q)}$ and $\Psi_{i}^{r, s} \mid p^{q} W \times p^{m} W=\Psi_{i}^{q, m}$. Then these two systems of polynomials may be used to construct a topological ring scheme taking values in any perfect $k$-algebra. The polynomials, $\Phi_{i}^{(r)}$, do not involve $p$ 'th roots. Consequently, a non-perfect topological additive group scheme may be defined. The ring structure requires passage to the perfect completion. Proceed as follows.

Let $X_{\mathbb{Z}}$ be a set of polynomial variables indexed by the integers. Let $A^{+}$denote $k^{+}\left\langle X_{\mathbb{Z}}\right\rangle$ and let $A_{\infty}^{+}$denote its perfect completion. Then, for fixed $i$, the sequences of polynomials, $\Phi_{i}^{(r)}\left(x_{r} \otimes 1, \ldots, x_{i} \otimes 1 ; 1 \otimes x_{i}, \ldots, 1 \otimes x_{r}\right)$ is a convergent sequence of polynomials in $A^{+} \hat{\otimes} A^{+}$while $\Psi_{i}^{r, s}\left(x_{r} \otimes 1, \ldots, x_{i-s} \otimes 1 ; 1 \otimes x_{s}, \ldots, 1 \otimes x_{i-r}\right)$ is a convergent sequence in $A_{\infty}^{+} \hat{\otimes} A_{\infty}^{+}$. Let $\hat{\Phi}_{i}$ and $\hat{\Psi}_{i}$ denote their limits in the corresponding complete tensor products. Then the mapping, $\alpha\left(x_{i}\right)=\hat{\Phi}_{i}$ gives $A^{+}$the structure of the coordinate ring of a commutative topological group scheme and $\alpha$ and $\mu\left(x_{i}\right)=\hat{\Psi}_{i}$ are maps giving $A_{\infty}^{+}$the structure of a formal ring scheme.

DEFINITION 11. The scheme of additive Witt fractions of $k$ is the formal $k$-scheme, $\operatorname{Spf}\left(A^{+}\right)$with the binary operation defined by $\alpha$. The scheme of Witt fractions is $\operatorname{Spf}\left(A_{\infty}\right)$ with the binary operations defined by the maps $\alpha$ and $\mu$ as, respectively, co-addition and comultiplication. We shall write $Q W_{k}^{+}$for the scheme of additive Witt fractions and $Q W_{k}$ for the scheme of Witt fractions.

It is of some significance to note that if $W_{k}$ denotes the Witt vectors, a multiplication of sorts can be defined on $p^{-r} W_{k}$ where this latter is an additive subgroup of $Q W_{k}^{+}$. The notation is self-explanatory.

Proposition 3. For $r, s \geq 0$ there is an algebraic bilinear map of group schemes, $\mu_{r, s}: p^{-r} W_{k}^{(s)} \times_{k} p^{-s} W_{k}^{(r)} \rightarrow p^{-r-s} W_{k}$. (Recall that the exponents in parentheses specify Frobenius covers.)

In view of the restriction formula, (2.4), no proof is required.

We include one more definition of an arithmetic nature. We recall the endomorphisms $V$ and $F$ of Serre [S1]. We must, for notational consistency rename them. Define two endomorphisms of algebras, both denoted $\sigma$, on $k^{+}\left\langle X_{\mathbb{Z}}\right\rangle$ and $k_{\infty}^{+}\left\langle X_{\mathbb{Z}}\right\rangle$, by the equation, $\sigma\left(x_{i}\right)=x_{i}^{p}$. We then extend this to the complete perfect closure of either ring where it becomes an automorphism. Each of the polynomials, $\Phi_{i}$ and $\Psi_{i}$ has coefficients in the integers, i.e., in $\mathbb{F}_{p}$, and hence $\Phi_{i}\left(x_{0}^{p}, \ldots, x_{i}^{p}\right)=\Phi_{i}\left(x_{0}, \ldots, x_{i}\right)^{p}$ and the same for $\Psi_{i}$. Written otherwise, this says that $\Phi_{i}$ and $\Psi_{i}$ satisfy the condition, $f\left(\sigma\left(x_{0}\right), \ldots, \sigma\left(x_{i}\right)\right)=\sigma\left(f\left(x_{0}, \ldots, x_{i}\right)\right)$ for all $i$. This however means that $\sigma$ behaves properly with respect to co-addition and co-multiplication on the scheme of Witt fractions, additive or otherwise, and so extends even to an automorphism of topological ring schemes on the complete Witt fractions and an automorphism of commutative group schemes in the additive case. Considering the effect of the contravariant morphism of Witt fractions, if $\sigma^{*}$ is the contravariant morphism, $\sigma^{*}\left(\sum\left(\tilde{\xi}_{i}\right)^{p^{-i}} p^{i}\right)=\sum\left(\tilde{\xi}_{i}^{p^{-1}}\right)^{p^{-i}} p^{i}$. That is to say, $\sigma^{*}$ is the classical Frobenius substitution of number theory, the topological 
generator of the Galois group of the Witt vectors. This is the morphism corresponding to $F$ in Serre [S1]. Notice however that it is an automorphism of the Witt fractions but only an endomorphism of the additive Witt fractions.

We also define a morphism, $\mathfrak{v}$, corresponding to $\mathfrak{v}\left(x_{i}\right)=x_{i-1}$. This is "vershiebung" and it is merely an additive morphism on either of the two schemes. Observe the well known fact that the composition $\sigma \circ \mathfrak{v}$ corresponds to multiplication by $p$.

Definition 12. The Frobenius substitution on $Q W_{k}$ and $Q W_{k}^{+}$is the endomorphism induced by the endomorphism of coordinate rings, $\sigma\left(x_{i}\right)=x_{i}^{p}$. The automorphism of the field or ring of points is written $\sigma^{*}$. It is an automorphism on the topological scheme $Q W_{k}$ but an endomorphism with non-reduced punctual fibers on $Q W_{k}^{+}$.

This definition can be extended, though not necessarily canonically, to other schemes derived from the Witt fractions such as the Vector space of dimension $n$ over the Witt fractions or the matrices over them. We shall always use $\sigma$ and $\sigma^{*}$ to represent these endomorphisms when no ambiguity will result.

Another remark is in order. One might wonder whether one must indeed pass to the perfect completion to define the Witt fractions. To verify that it is necessary just note that the result of multiplying the Witt vector whose only non-zero component is $\xi_{0}$ in degree zero with the one whose only non-zero component is $\eta_{r}$ in degree $r$ is the vector with the single non-zero component, $\xi_{0}^{p^{r}} \eta_{r}$ in degree $r$. In negative degree this requires arbitrary $p^{n}$ 'th roots.

Now $Q W_{k}$ is a ring object in the category of topological schemes whose set of $k$-points is $K$, the fraction field of $W_{k}$. Hence, for any topological scheme $Z$, $\operatorname{Hom}\left(Z, Q W_{k}\right)$ is a ring. Let $Z$ denote the $n$-fold product of $Q W_{k}$ and let $X_{i}$ denote projection on the $i$ 'th factor. Any $C \in K$ is a $k$-point of $Q W_{k}$, that is, a continuous homomorphism from the coordinate ring of $Q W_{k}$ to $k$. The composition of this map with the algebra inclusion of $k$ in the coordinate ring of $Q W_{k}$ is contravariant to the map from $Q W_{k}$ to $Q W_{k}$ which sends every point to the constant value $C$. We call this the constant map associated to $C$. Identify $C \in K$ with the associated constant map, $C: Q W_{k} \rightarrow Q W_{k}$. One may then take arbitrary polynomials in the $X_{i}$ and these are maps from $Q W_{k}^{n}$ to $Q W_{k}$ in the category of topological schemes. Since relative fiber products and hence fibers of morphisms exist in the category of topological schemes, it follows that any affine scheme of finite type over $Q W_{k}$ may be viewed as an affine topological scheme over $k$. (Affine because the fibre product of affines is affine.) It is also clear that a morphism of affine schemes of finite type over $Q W_{k}$ is a morphism in the category of topological schemes. From this one can conclude that any scheme of finite type over $Q W_{k}$ affine or not admits a structure as a topological scheme over $k$. It is not immediately clear that these procedures are functorial or indeed that they give a uniquely defined structure in the appropriate category. This ambiguity is a consequence of the fact that these schemes, as we have defined them, do not have a precise definition as something like Greenberg functors or limits of Greenberg functors. 
2.6. Localized Greenberg functors: We begin this section with an observation. Suppose that $X$ is a $k$-scheme and that $B$ is a $k$-algebra. Then one may construct a presheaf on $X$ by setting $\mathcal{B}(U)=\operatorname{Hom}_{k}\left(B, \mathcal{O}_{X}(U)\right)$ for any open set $U$ in $X$.

LEMMA 1. For any $k$-scheme $X$ and k-algebra, B, $\mathcal{B}$ is a sheaf of sets.

Proof. Suppose $U=\bigcup_{i} M_{i}$ is an open covering and let $\alpha, \beta \in \mathcal{B}(U)$ be two sections such that $\alpha\left|M_{i}=\beta\right| M_{i}$ for each $i$. This means that $\alpha(b)\left|M_{i}=\beta(b)\right| M_{i}$ for each $i$ and hence $\alpha(b)=\beta(b)$ for each $b \in B$. That is, $\alpha=\beta$.

Similarly suppose that $\alpha_{i} \in \mathcal{B}\left(M_{i}\right)$ is a family of sections such that $\alpha_{i} \mid\left(M_{i} \cap M_{j}\right)=$ $\alpha_{j} \mid\left(M_{i} \cap M_{j}\right)$ for each $i, j$. This means that for any $b \in B, \alpha_{i}(b)\left|\left(M_{i} \cap M_{j}\right)=\alpha_{j}(b)\right|\left(M_{i} \cap\right.$ $M_{j}$ ). Hence there is a section $\alpha(b) \in \mathcal{O}_{X}(U)$ restricting to the sections $\alpha_{i}(b)$. (Said otherwise, $\operatorname{Hom}\left(B_{X}, \mathcal{O}_{X}\right)$ is a sheaf where $B_{X}$ denotes the constant sheaf.)

We recall the classic results of Greenberg. Let $\mathfrak{W}$ be a ring scheme over $k$ which is an inverse limit of finite ring schemes each isomorphic as a scheme to an affine space and let $W=\mathfrak{W}(k)$. For a $k$-scheme, $Y$, define a ringed space by taking the underlying space of $Y$ as its topological space. Construct a presheaf by letting $\mathcal{W}_{Y}(U)=\mathfrak{W}\left(\mathcal{O}_{Y}(U)\right)$. By Lemma 1 , this is a sheaf. The underlying space of $Y$ with the sheaf, $\mathcal{W}_{Y}$ will be denoted $\mathfrak{W}(Y)$. The Greenberg functor, $\mathcal{G}_{0}$ associated to $\mathfrak{W}$ is the right adjoint of $\mathfrak{W}$, that is, for each Spec $W$ scheme, $Z$, and $k$-scheme, $X$, the equation, $\operatorname{Hom}_{k}\left(\mathcal{G}_{0} Z, X\right)=\operatorname{Hom}_{\operatorname{Spec}} W(Z, \mathfrak{W}(X))$ holds functorially in $Z$ and $X$. It exists and is defined on the category of schemes of finite type over $W$ and in fact in a somewhat more general context which is of no relevance to us here ([MJG1], [MJG2]). Our purpose here is to show that a localized version of the Greenberg functor exists. That is, there is an analogue of the Greenberg functor defined on schemes of finite type over the fraction field of $W$. It is a topological scheme over $k$ and it does not have all the properties of the classical Greenberg functor.

To facilitate our discussion I would like to introduce certain assumptions. A ring scheme $\mathfrak{W}$ on the category of $k$-schemes for some perfect field, $k$, will be called a $w$-scheme if it satisfies the following list of conditions which essentially codify some common properties of formal power series and Witt vectors:

HYPOTHESES 1. A w-scheme over $k$ is a ring scheme assumed to satisfy the following:

(1) The set of $\mathrm{Spec} A$-valued points of $\mathfrak{W}$ is isomorphic to the sequences, $\left(a_{0}, a_{1}, \ldots\right)$ indexed by the positive integers and multiplication and addition are given by sequences of polynomials in these entries.

(2) The map $\eta(a)=(a, 0,0, \ldots)$ is multiplicative and the map $\phi\left(a_{0}, a_{1}, \ldots\right)=a_{0}$ is a homomorphism of rings. For a perfect ring, A, the kernel of $\phi$ is principal with generator $(0,1,0,0, \ldots)=\pi$. For any $A$ the zero of $\mathfrak{W}(A)$ is the zero sequence, $(0,0, \ldots)$.

(3) For any $k$-algebra, $A$, and elements $a_{i}, b_{j} \in A$, the product

$$
\left(0,0, \ldots, 0, a_{i}, a_{i+1}, \ldots\right)\left(0,0, \ldots 0, b_{j}, b_{j+1}, \ldots\right)
$$


is an A point of $\mathfrak{W}$ whose first non-vanishing entry is $a_{i}^{p^{j}} b_{j}^{p^{i}}$ in the $(i+j)$ 'th place, where $p$ is the characteristic exponent of $k$ (i.e., one in characteristic 0$)$. Moreover, for any $c \in A$ and any $\mathbf{a}=\left(a_{0}, a_{1}, \ldots\right) \in \mathfrak{W}(A), \eta(c) \mathbf{a}=\left(c a_{0}, c^{p} a_{1}, \ldots, c^{p^{i}} a_{i}, \ldots\right)$.

(4) For each integer, $n$, the assignment, $I_{n}(A)=\left\{\left(a_{0}, a_{1}, \ldots\right) \in \mathfrak{W}(A): a_{0}=a_{1}=\right.$ $\left.\cdots=a_{n-1}=0\right\}$, defines a scheme of ideals and the quotient $\mathfrak{W}(A) / I_{n}(A)=\mathfrak{W}_{n}(A)$ is a finite ring-scheme with functor of points isomorphic as a set to the sequences $\left(a_{0}, \ldots, a_{n-1}\right)$. The map from $\mathfrak{W}(A)$ given by simple truncation is the quotient homomorphism.

(5) There is an endomorphism of the identity functor on the category of $k$-algebras, $\sigma$, and an additive map, $\mathfrak{v}: \mathfrak{W}(A) \rightarrow \mathfrak{W}(A)$ given by $\mathfrak{v}\left(a_{0}, a_{1}, \ldots\right)=\left(0, a_{0}, a_{1}, \ldots\right)$ (the shift), so that $V \circ \mathfrak{W}(\sigma)$ is the same as multiplication by $\pi$.

These hypotheses have certain immediate consequences. Let $\mathfrak{q}$ be an ideal in the $k$ algebra, $A$. Then the natural morphism, $A \rightarrow A / \mathfrak{q}$ by functoriality induces a morphism $\mathfrak{W}(A) \rightarrow \mathfrak{W}(A / \mathfrak{q})$. Write $\mathfrak{W}(\mathfrak{q})$ for the kernel of this latter homomorphism. It is selfevidently the set of sequences, $\left(a_{0}, a_{1}, \ldots\right)$ such that for each $i, a_{i} \in \mathfrak{q}$. Finally, $I_{n}(A)$, the sequences whose first $n$ coordinates are 0 is an ideal in $\mathfrak{W}(A)$ for each $n \geq 1$ and $\mathfrak{W}(A)$ is a complete topological algebra with these ideals as neighborhoods of 0 . For $n \geq 1$, by the third hypothesis, $I_{n}(A)$ consists of topological nilpotents.

1. For any domain, $A, \mathfrak{W}(A)$ is a domain.

To see this let $\mathbf{a}$ and $\mathbf{b}$ be elements of $\mathfrak{W}(A)$. If they are both non-zero let $a_{i}$ and $b_{j}$ in the $i$ 'th and $j$ 'th places be their initial non-vanishing coordinates. By Hypotheses $1,(2)$, the initial term of $\mathbf{a b}$ is $a_{i}^{p^{j}} b_{j}^{p^{i}}$ which must also be non-zero.

2. For any $k$-algebra, $A$, and any prime $\mathfrak{q} \subseteq A, \mathfrak{W}(\mathfrak{q})$ is prime in $\mathfrak{W}(A)$.

This follows immediately from 1.

3. For any $k$-algebra, $A$, and any $\mathbf{a}=\left(a_{0}, a_{1}, \ldots\right) \in \mathfrak{W}(A)$, the complete localization, $\mathfrak{W}(A)_{\langle a\rangle}$ is isomorphic to $\mathfrak{W}\left(A_{a_{0}}\right)$.

The proof of this is not entirely trivial. First we prove that if $\tilde{a}_{0}=\eta\left(a_{0}\right)$ then $\mathfrak{W}(A)_{\langle\mathbf{a}\rangle}=$ $\mathfrak{W}(A)\left\langle\tilde{a}_{0}\right\rangle$. To see this, note that $\mathbf{a}-\tilde{a}_{0}$ is topologically nilpotent in both rings. Hence, in $\mathfrak{W}(A)_{\tilde{a}_{0}}, \tilde{a}_{0}^{-1}\left(\mathbf{a}-\tilde{a}_{0}\right)=u$ is as well. Hence $1+u$ and so also $\tilde{a}_{0}(1+u)=\mathbf{a}$ are both units. Consequently there is a natural map from $\mathfrak{W}(A)_{\mathbf{a}}$ to $\mathfrak{W}(A)_{\tilde{a}_{0}}$ which composes with the natural injection of $\mathfrak{W}(A)$ into $\mathfrak{W}(A)_{\text {a }}$ to give the natural injection into $\mathfrak{W}(A)_{\tilde{a}_{0}}$. In $\mathfrak{W}(A)_{\mathbf{a}}$ the element $\mathbf{a}-\tilde{a}_{0}$ is topologically nilpotent and so $\mathbf{a}^{-1}\left(\mathbf{a}-\tilde{a}_{0}\right)$ is. One deduces that $\tilde{a}_{0}$ is invertible in this ring and so there is a natural map $\mathfrak{W}(A)_{\left\langle\tilde{a}_{0}\right\rangle} \rightarrow \mathfrak{W}(A)_{\langle\mathbf{a}\rangle}$ which is compatible with the natural injections. Thus the two are isomorphic.

Now we can prove that $\mathfrak{W}(A)\left\langle\tilde{a}_{0}\right\rangle$ is isomorphic to $\mathfrak{W}\left(A_{a_{0}}\right)$. There is a natural map from $\mathfrak{W}(A)$ into $\mathfrak{W}\left(A_{a_{0}}\right)$ and this map clearly carries $\tilde{a}_{0}$ to an invertible element. Hence there is a unique map from $W(A)_{\left\langle\tilde{a}_{0}\right\rangle}$ extending the natural map. Next notice that for any finite sequence, $\mathbf{b}=\left(b_{0} / a_{0}^{q_{0}}, b_{1} / a_{0}^{q_{1}}, \ldots, b_{n} / a_{0}^{q_{n}}, 0,0, \ldots\right)$ there is a finite integer, $N$, so that $p^{i} N>q_{i}$ for all $i \leq n$. By 23 iii), it follows that $\tilde{a}_{0}^{N} \mathbf{b}$ is in the image of $\mathfrak{W}(A)$ and 
so $\mathbf{b}$ is in the image of $\mathfrak{W}(A)_{\left\langle\tilde{a}_{0}\right\rangle}$. The finite elements are evidently dense and so the map is surjective. For injectivity, notice that if a $\mathbf{c} \in \mathfrak{W}(A)$ is taken to zero, then there is a sequence $N_{i}$ so that $\tilde{a}_{0}^{N_{i}} \mathbf{c} \in I_{i}(A)$. But this says that 0 is in the closure of the set of elements, $\tilde{a}_{0}^{N_{i}} \mathbf{c}$. Hence the image of $\mathbf{c}$ in $\mathfrak{W}(A)_{\left\langle\tilde{a}_{0}\right\rangle}$ is already null which proves what we wished to prove.

Without proof, we include the following whose proof is very close in spirit to the last part of the proof above. Both proofs amount to the observation that the functors involved (tensor product, localization) are in principle preserved by the finite schemes of which $\mathfrak{W}$ is the inverse limit and then passing to the limit.

$$
\text { 4. } \mathfrak{W}\left(A \otimes_{k} B\right)=\mathfrak{W}(A) \hat{\otimes}_{W} \mathfrak{W}(B) .
$$

Consider the functor $\mathfrak{W}$, defined in the first paragraph of this section, from the category of $k$-schemes to the category of local ringed spaces over $W$. Its value on the scheme, $X$, has the same underlying space but its sheaf, which we write $\mathcal{W}_{X}$ is the sheaf $U \mapsto \mathfrak{W}\left(\mathcal{O}_{X}(U)\right)$. This ringed space is $\mathfrak{W}(X)$.

Proposition 4. Let $X=\operatorname{Spec}(A)$ where $A$ is a k-algebra. Then $\mathfrak{W}(X)=\operatorname{Spf} \mathfrak{W}(A)$. Moreover $\mathfrak{W}(X)$ is a topological $\mathfrak{W}(k)$-scheme for each $k$-scheme $X$.

Proof. The open primes of $\mathfrak{W}(A)$ are just those containing $I_{1}(A)$. Hence they are exactly the primes of $\mathfrak{W}(A) / I_{1}(A)=A$. The topology is clearly the same. Hence the underlying space of $\operatorname{Spf}(\mathfrak{W}(A))$ is exactly the underlying space of $\operatorname{Spec}(A)$. The sheaf is determined by its values on a subbase for the topology, hence for sets $D(\mathbf{a})$. By $3, D(\mathbf{a})=$ $D\left(\tilde{a}_{0}\right)$ and the value of the structure sheaf on $D\left(\tilde{a}_{0}\right)$ is just $\mathfrak{W}\left(A_{a_{0}}\right)$. Hence the underlying spaces of the two ringed spaces coincide and the structure sheaves coincide as well. Write $X$ as a union of affines, $\operatorname{Spec}\left(A_{i}\right)$. It follows that $\mathfrak{W}(X)=\bigcup_{i} \operatorname{Spf}\left(\mathfrak{W J}\left(A_{i}\right)\right)$.

Thus using the notion of a topological scheme as we have defined it, the functor, $\mathfrak{W}$, which naturally occurs in the definition of a Greenberg functor actually carries schemes to topological schemes. Before proceeding we recall a particular universal mapping property of local ringed spaces. Recall that given a local ringed space $\tilde{M}=\left(M, \mathcal{O}_{M}\right)$ then for any map, $\phi^{0}$, from a commutative ring, $R$, to $\Gamma\left(\tilde{M}, \mathcal{O}_{M}\right)$ there is an induced map, $\phi: \tilde{M} \rightarrow \operatorname{Spec}(R)$. At the point, $x$, the map is determined by composing $\phi^{0}$ with the natural map from $\Gamma\left(\tilde{M}, \mathcal{O}_{M}\right)$ to the germs, $\mathcal{O}_{M, x}$, and using the resulting composition to take the inverse image of the maximal ideal at $x$. There is such a map for $R=\Gamma\left(\tilde{M}, \mathcal{O}_{M}\right)$, from $\tilde{M}$ to $\operatorname{Spec}\left(\Gamma\left(\tilde{M}, \mathcal{O}_{M}\right)\right)$ and this map is universal in that every map from $\tilde{M}$ to an affine scheme factors uniquely through this map via the natural map contravariant to the map of global sections, $R \rightarrow \Gamma\left(\tilde{M}, \mathcal{O}_{M}\right)$. Said otherwise, the functor, Spec, from the category of commutative rings with unit to the category of local ringed spaces and local morphisms, is a contravariant right adjoint to the functor global sections in the structure sheaf. The statement just for schemes rather than local ringed spaces is exercise 2.4 of [Ha] but it trivially extends to local ringed spaces. It is proven for local ringed spaces in [EGA, I, p. 310, Proposition 1.6.3]. 
For a ring, $A$, let $A^{p^{-\infty}}$ denote the incomplete perfect cover of $A$, that is, the limit, $\lim _{n} A^{p^{-n}}$. (See the fourth paragraph of Subsection 2.2). This ring is the ring of regular

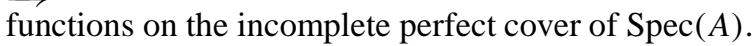

Definition 13. Assume that $\mathfrak{W}$ is w-scheme, i.e., that it satisfies the hypotheses, 23. The localized w-functor associated to $\mathfrak{W}$ is the functor, $\mathfrak{W}_{\pi}$, which associates to each commutative $k$-algebra, $A$, the value of $\mathfrak{W}$ on $A^{p^{-\infty}}$ localized at the uniformizing parameter, $\pi$. That is, $\mathfrak{W}_{\pi}(A)=\left(\mathfrak{W}\left(A^{p^{-\infty}}\right)\right)_{\pi}$. The functor, $\mathfrak{W}_{\pi}$, from the category of $k$-schemes to the category of $K$-ringed spaces assigns to each $k$-scheme, $X$, the ringed space, $\mathfrak{W}_{\pi}(X)$, which has the same underlying topological space as $X$. Its sheaf is that associated to the presheaf,

$$
\mathfrak{W}_{\pi}\left(\mathcal{O}_{X}\right)(U)=\left(\mathfrak{W}_{\pi}\left(\mathcal{O}_{X}(U)\right) .\right.
$$

LEMMA 2. For any $k$-scheme, $X, \mathfrak{W}_{\pi}\left(\mathcal{O}_{X}\right)$ is a sheaf.

ProOF. First observe that $\mathcal{O}_{X}^{p^{-\infty}}$ is a sheaf. It follows that $\mathfrak{W}\left(\mathcal{O}_{X}^{p^{-\infty}}\right)$ is also a sheaf, since it is simply a product of a countable number of copies of $\mathcal{O}_{X}^{p^{-\infty}}$.

Now we show that $\mathfrak{W}_{\pi}\left(\mathcal{O}_{X}\right)$ is a sheaf. Let $\left\{U_{i}\right\}_{i \in I}$ be a covering indexed by the ordered set $I$ and for $i<j$ let $U_{i, j}=U_{i} \cap U_{j}$. Let $j_{1}, j_{2}: \prod_{i} \mathfrak{W} \mathfrak{J}\left(\mathcal{O}_{X}^{0}\left(U_{i}\right)\right) \rightarrow \prod_{i<j} \mathfrak{W}\left(\mathcal{O}_{X}^{p^{-\infty}}\left(U_{i, j}\right)\right)$ be the maps defined by $j_{1}\left(\left\{s_{i}\right\}_{i}\right)=\left\{s_{i} \mid U_{i, j}\right\}_{i<j}, j_{2}\left(\left\{s_{i}\right\}_{i}\right)=\left\{s_{i} \mid U_{j, i}\right\}_{j<i}$. That $\mathfrak{W}\left(\mathcal{O}_{X}^{p^{-\infty}}\right)$ is a sheaf is equivalent to the exactness of:

$$
0 \rightarrow \prod_{i} \mathfrak{W}\left(\mathcal{O}_{X}^{p^{-\infty}}\left(U_{i}\right)\right) \stackrel{j_{1}-j_{2}}{\longrightarrow} \prod_{i<j} \mathfrak{W}\left(\mathcal{O}_{X}^{p^{-\infty}}\left(U_{i, j}\right)\right)
$$

for each covering, $\left\{U_{i}\right\}_{i \in I}$. For any open $M, \mathfrak{W}_{\pi}\left(\mathcal{O}_{X}\right)(M)=\mathfrak{W}\left(\mathcal{O}_{X}^{p^{-\infty}}(M)\right)_{\pi}$ and consequently one may localize the exact sequence (2.7) to obtain an exact sequence:

$$
0 \rightarrow \prod_{i} \mathfrak{W}_{\pi}\left(\mathcal{O}_{X}\right)\left(U_{i}\right) \stackrel{j_{1}-j_{2}}{\longrightarrow} \prod_{i<j} \mathfrak{W}_{\pi}\left(\mathcal{O}_{X}\right)\left(U_{i, j}\right) .
$$

The exactness of (2.8) for any covering is exactly the statement that $\mathfrak{W}_{\pi}\left(\mathcal{O}_{X}\right)$ is a sheaf. It also should be noted that the stalks of $\mathfrak{W}_{\pi}\left(\mathcal{O}_{X}\right)$ are local. To see this one need only apply the functor $\mathfrak{W}_{\pi}$ to the evaluation map, $\mathcal{O}_{X}(U) \rightarrow \mathcal{O}_{X, x} / \mathfrak{m}_{x}$.

Definition 14. Let $X$ be a $K$-scheme. Then a localized Greenberg scheme associated to $X$ is a topological $k$-scheme, $\mathcal{G} X$, satisfying the functorial equation $\operatorname{Hom}_{K}\left(\mathfrak{W}_{\pi}(Y), X\right)=$ $\operatorname{Hom}_{k}(Y, \mathcal{G} X)$ for all $k$-schemes, $Y$.

Clearly, since the functor which $\mathcal{G} X$ represents is determined, if a topological scheme representing it exists, it is unique up to a unique isomorphism. We shall prove that it exists for all schemes of finite type over $K$ and that $\mathcal{G}$ behaves well with respect to relative fiber products. Once existence is established for schemes of finite type over $K$, it follows that $\mathcal{G}$ is in fact a covariant functor from schemes of finite type over $K$ to topological schemes over $k$. The universal mapping property of $\operatorname{Spec}\left(\Gamma\left(X, \mathcal{O}_{X}\right)\right)$ alluded to above plays a crucial role.

REMARK 1. It is natural to attempt to construct these objects without passing to perfect closures. However even the simplest polynomials of positive degree such as $x^{2}$ or $x y$ involve 
arbitrarily large $p$ 'th roots when they are expressed in $k$ coordinates. It follows that one must use a perfect completion to adequately describe the coordinate ring even of $\mathcal{G} \mathbb{A}_{k}^{1}$. The use of a perfect coordinate ring then forces the use of the perfect closure in the definition of $\mathfrak{W}_{\pi}$.

Lemma 3. Let $X, Y$ and $Z$ be affine $K$-schemes and $f: X \rightarrow Z$ and $g: Y \rightarrow Z$ be $K$ morphisms. Let $M$ be a local ringed space over $K$. Then

$$
\operatorname{Hom}_{K}\left(M, X \times_{Z} Y\right)=\operatorname{Hom}_{K}(M, X) \times_{\operatorname{Hom}_{K}(M, Z)} \operatorname{Hom}_{K}(M, Y) .
$$

Proof. This is simply so because for any affine $K$-scheme, $T$,

$$
\operatorname{Hom}_{K}(M, T)=\operatorname{Hom}_{K}\left(\operatorname{Spec}\left(\Gamma\left(M, \mathcal{O}_{M}\right), T\right) .\right.
$$

Taking $T$ equal to the desired product yields the result.

LEMMA 4. Let $X$ and $Y$ be two topological schemes over $k$. Then if there is an isomorphism of functors, $\operatorname{Hom}_{k}(, X) \simeq \operatorname{Hom}_{k}(, Y)$, on the category of ordinary, affine $k$-schemes then $X$ and $Y$ are canonically isomorphic.

Proof. Any topological $k$ scheme, $Z$, can be represented as an inductive limit, $Z=\lim _{i \in I} Z_{i}$ where the $Z_{i}$ are closed discrete subschemes. Hence $\operatorname{Hom}_{k}(Z, X)=$ $\lim _{i \in I} \overrightarrow{\operatorname{Hom}}_{k}\left(Z_{i}, X\right)$ and the same for $Y$. Consequently the isomorphism of functors extends to the full category of topological schemes over $k$. Thus one must only verify that the two functors are isomorphic on the category of $k$-schemes. Since every scheme can be represented as the coequalizer of two morphisms from one disjoint union of affines to another, if the two functors are isomorphic on the category of affine $k$-schemes, they are isomorphic on the category of all $k$-schemes. Hence the result follows at once by Yoneda's Lemma.

We slightly extend the notation of Definition 6. Let $\mathbb{Z}^{n}$ denote the index set of pairs, $(i, j)$ where $i, j \in \mathbb{Z}, 1 \leq i \leq n$. Then $k^{+}\left\langle X_{\mathbb{Z}^{n}}\right\rangle$ denotes the completion of the ring of polynomials in a set of indeterminates indexed by $\mathbb{Z}^{n}$. The topology is determined by taking the ideals, $I_{r}$, as a subbase where $I_{r}$ is the ideal generated by all $x_{i, j}$ with $j<r$. A subscript $\infty$ on the right bracket indicates perfect completion and when $n=1$ we use a single subscript in $\mathbb{Z}$.

Lemma 5. Let $X$ be locally closed in $\mathbb{A}_{K}^{n}$. That is, $X$ is an open subset of a $K$-closed subset of $\mathbb{A}_{K}^{n}$. Let $j: U \rightarrow X$ be an open embedding. Then there are localized Greenberg functors, $\mathcal{G} X$ and $\mathcal{G} U$ and moreover the map $\mathcal{G} U \rightarrow \mathcal{G} X$ induced by $j$ is an open embedding of topological perfect schemes.

Proof. Let $A_{n, \infty}^{+}=k^{+}\left\langle X_{\mathbb{Z}^{n}}\right\rangle_{\infty}$ and let $A_{\infty}^{+}=k^{+}\left\langle X_{\mathbb{Z}}\right\rangle_{\infty}$. We prove that $\mathcal{G} \mathbb{A}_{K}^{n}=$ $\operatorname{Spf}\left(A_{n, \infty}^{+}\right)$. Then $\operatorname{Hom}_{k}\left(\operatorname{Spec} R, \operatorname{Spf}\left(A_{n, \infty^{+}}\right)\right)$is the set of continuous algebra homomorphisms from $A_{n, \infty}^{+}$to $R$. By Proposition 2 and the remarks in Subsection 2.2, these homomorphisms correspond to the $n$-tuples of sequences $\left(x_{i, j}\right)_{j \in \mathbb{Z}}, i=1, \ldots, n$ with $x_{i, j} \in R_{0}$ and $x_{i, j}=0$ for all sufficiently small $j$. This set is, by Definition 13 and Proposition 2 , in bijective correspondence with the set of $n$-tuples of elements of $\mathfrak{W}_{\pi}(R)$. These $n$ tuples are the $\mathfrak{W}_{\pi}(R)$ points of $\mathbb{A}_{K}^{n}$. This establishes that $\operatorname{Hom}_{k}\left(\operatorname{Spec}(R), \operatorname{Spf} A_{n, \infty}^{+}\right)=$ 
$\operatorname{Hom}_{K}\left(\mathfrak{W}_{\pi}(\operatorname{Spec}(R)), \mathbb{A}_{K}^{n}\right)$ for all $k$-algebras. By Lemma 4 , this shows that $\operatorname{Spf}\left(A_{n, \infty}^{+}\right)=$ $\mathcal{G} \mathbb{A}_{K}^{n}$.

Now think of $\operatorname{Spf}\left(A_{\infty}^{+}\right)$as the topological ring scheme of Witt fractions. The projections of $\operatorname{Spf}\left(A_{n, \infty}^{+}\right)$on the $i$ 'th factor, which we write $\tilde{X}_{i}$, are points of the Witt fractions with values in $\mathcal{G} \mathbb{A}_{K}^{n}$. Under the correspondence of the previous paragraph, this mapping corresponds to the $i$ 'th coordinate function. Since these are points in a ring scheme, any polynomials in $n$ variables over $K$ can be evaluated on them. Under the functorial correspondence of points $F\left(\tilde{X}_{1}, \ldots, \tilde{X}_{n}\right)$ will correspond exactly to the polynomial, $F$, regarded as a map from $n$ space to the line. Further, $F$ is by its very definition an ind-algebraic map from $\operatorname{Spf}\left(A_{n, \infty}^{+}\right)=$ $\mathcal{G} \mathbb{A}_{K}^{n}$ to $\operatorname{Spf} A_{\infty}^{+}=\mathcal{G} \mathbb{A}_{K}^{1}$. Consequently, given any two sets of polynomials in $n$ variables, $F_{1}, \ldots, F_{r}$ and $H_{1}, \ldots, H_{s}$, the complement of the zeros of the $H_{i}$ in the common zeros of the $F_{i}$ can be realized as a locally closed topological subscheme of $\mathcal{G} \mathbb{A}_{K}^{n}$, namely, as $\bigcap_{i=1}^{r} F_{i}^{-1}(0) \cap \bigcap_{i=1}^{s} H_{i}^{-1}\left(\mathcal{G} \mathbb{A}_{K}^{1} \backslash\{0\}\right)$. This constructs a localized Greenberg functor for $X$. To prove the statement about the open embedding $U \subseteq X$ just note that to define $U$ we need only add a finite number of polynomials to the $H_{i}$.

Lemma 6. Let $\phi: X \rightarrow Z$ and $\psi: Y \rightarrow Z$ be two open embeddings of $K$ schemes. Assume that a localized Greenberg functor for each of these three schemes exists and denote these $\mathcal{G} X, \mathcal{G} Y$ and $\mathcal{G} Z$, respectively. Then the relative fiber product, $X \times{ }_{Z} Y$ admits a localized Greenberg functor and $\mathcal{G} X \times{ }_{\mathcal{G} Z} \mathcal{G} Y=\mathcal{G}\left(X \times{ }_{Z} Y\right)$.

Proof. Let $T$ be a $k$-scheme. By the definition of relative fiber products,

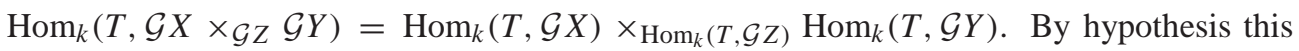
is equal to $\operatorname{Hom}_{K}\left(\mathfrak{W}_{\pi}(T), X\right) \times_{\operatorname{Hom}_{K}}\left(\mathfrak{W}_{\pi}(T), Z\right) \operatorname{Hom}_{K}\left(\mathfrak{W}_{\pi}(T), Y\right)$. This fiber product consists of the pairs of local ringed space morphisms, $(f, g)$, such that $\phi \circ f=\psi \circ g$. As these are open embeddings, this says that the image of $T$ under either of the morphisms is in the intersection of the two open subschemes and that the values of $f$ and $g$ on any point coincide. Hence the last fiber product of Hom's is equal to $\operatorname{Hom}_{K}\left(\mathfrak{W}_{\pi}(T), \phi(X) \cap \psi(Y)\right)$. This last intersection however is none other than $X \times_{Z} Y$. That is, we have shown that $\operatorname{Hom}_{k}(T, \mathcal{G} X \times \mathcal{G Z} \mathcal{G} Y)=\operatorname{Hom}_{K}\left(\mathfrak{W}_{\pi}(T), X \times{ }_{Z} Y\right)$.

THEOREM 1. Let $X$ be a scheme of finite type over $K$. Then a localized Greenberg scheme, $\mathcal{G} X$, exists in the category of topological schemes over $k$.

Proof. Represent $X$ as a union, $X=\bigcup_{i \in I} U_{i}$ for an ordered set, $I$. For $i<j$ write $U_{i} \cap U_{j}=U_{i, j}$ and define $U_{i, j, r}$ similarly for $i<j<r$. Then there are three maps $q_{i, j}: \bigsqcup_{r, s, t} U_{r, s, t} \mapsto \bigsqcup_{r, s} U_{r, s} i, j=1,2,3, i<j$. Then $q_{2,3}$ maps $U_{r, s, t}$ to $U_{s, t}, q_{1,3}$ maps it to $U_{r, t}$ and $q_{1,2}$ maps it to $U_{r, s}$. Define maps, $q_{i}: \bigsqcup_{r, s} U_{r, s} \rightarrow U_{r}$. Let $X^{1}=\bigsqcup_{r} U_{r}$ and let $X^{2}=\bigsqcup_{r, s} U_{r, s}$. Then $X$ is the co-equalizer of the two maps, $q_{i}: X^{1} \rightarrow X^{2}$ and it may be constructed as a topological space and a ringed space whenever the following compatibility condition holds. To describe it we consider the following three diagrams: 

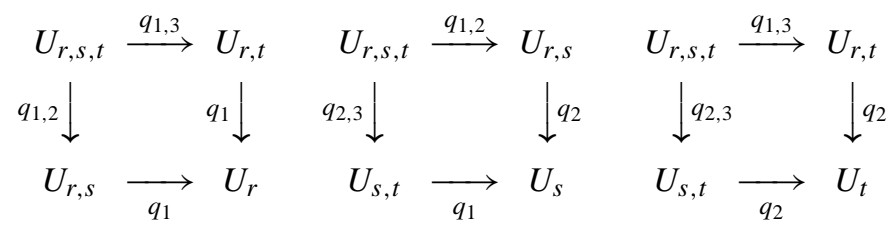

Then the necessary compatibility condition is just that, for any triple, $r, s, t$, these three squares are Cartesian which in the case of open embeddings just means that $U_{r, s, t}$ is the intersection of the sets on the upper right and the lower left in the set at the lower right. Each of these schemes is locally closed in $\mathbb{A}_{K}^{n}$ for some $n$ and so admits a local Greenberg functor. Hence one may apply the functor, $\mathcal{G}$ to each of the schemes and morphisms in the three rectangles of the diagram above and by Lemma 6 each square remains Cartesian. Further by Lemma 5 each of the maps involved is an open embedding. It follows that one may glue the topological schemes, $\mathcal{G} U_{i}$, along the subschemes, $\mathcal{G} U_{i, j}$, to obtain a topological scheme $\tilde{X}$. What must be shown is that $\tilde{X}$ is a localized Greenberg scheme for $X$. We may write $\tilde{X}=\bigcup_{i} \mathcal{G} U_{i}, \mathcal{G} U_{i} \cap \mathcal{G} U_{j}=\mathcal{G} U_{i, j}$. We must show that $\operatorname{Hom}_{k}(T, \tilde{X})=\operatorname{Hom}_{K}\left(\mathfrak{W}_{\pi}(T), X\right)$.

First suppose that $f: \mathfrak{W}_{\pi}(T): \rightarrow X$ is a morphism of local ringed spaces. Then by the Hypotheses 1 and Proposition $4, f^{-1}\left(U_{i}\right)$ is open in $T$ and this set together with the sheaf, $\left.\mathfrak{W}_{\pi}\left(\mathcal{O}_{T}\right)\right|_{f^{-1}\left(U_{i}\right)}$, is just $\mathfrak{W}_{\pi}\left(f^{-1}\left(U_{i}\right)\right)$ and the same for $U_{i, j}$. Hence $f$ restricts to give a family of maps, $f_{i}: \mathfrak{W}_{\pi}\left(f^{-1}\left(U_{i}\right)\right) \rightarrow U_{i}$ which agree on the intersections which are the spaces $\mathfrak{W}_{\pi} f^{-1}\left(U_{i, j}\right)$. Let $T_{i}$ and $T_{i, j}$ be open subschemes of $T$ corresponding to the sets $f^{-1}\left(U_{i}\right)$ and $f^{-1}\left(U_{i, j}\right)$. Then, since the schemes, $U_{i}$ and $U_{i, j}$ admit localized Greenberg schemes, the maps $f_{i}$ and $f_{i, j}$ correspond to a compatible family of maps $\bar{f}_{i}: T_{i} \rightarrow \mathcal{G} U_{i}$. These determine a map from $T$ to $\tilde{X}$ which is after all the scheme obtained by glueing the $\mathcal{G} U_{i}$ along the $\mathcal{U}_{i, j}$.

Conversely, let $\phi: T \rightarrow \tilde{X}$ be a morphism of topological $k$-schemes. Let $T_{i}=$ $\phi^{-1}\left(\mathcal{G} U_{i}\right)$. Restricting $\phi$ to $T_{i}$ gives a unique maps which correspond, by adjointness, to a map, $f_{i}: \mathfrak{W}_{\pi}\left(T_{i}\right) \rightarrow U_{i}$, for each $i$, and functoriality forces these to be a compatible family which hence piece together to a map, $f: \mathfrak{W}_{\pi}(T) \rightarrow X$. This shows that $\tilde{X}$ is a localized Greenberg scheme for $X$.

THEOREM 2. Let $X, Y$ and $Z$ be three schemes of finite type over $K$ and let $\phi: X \rightarrow Z$ and $\psi: Y \rightarrow Z$ be two $K$-morphisms. Then there is a canonical isomorphism, $\mathcal{G}\left(X \times_{Z} Y\right) \simeq$ $\mathcal{G} X \times{ }_{\mathcal{G} Z} \mathcal{G} Y$.

Proof. Write $Z$ as a union of affines, $Z_{i}$, and write $\phi^{-1}\left(Z_{i}\right)$ and $\psi^{-1}\left(Z_{i}\right)$ as unions of affines, $X_{i, r}$ and $Y_{i, s}$. Then $X \times{ }_{Z} Y$ is the union of affines $\bigcup_{i, r, s} X_{i, r} \times{ }_{Z_{i}} Y_{i, s}$ and hence $\mathcal{G}\left(X \times_{Z} Y\right)$ is, by the argument of the previous proof, equal to the union of the open sets $\mathcal{G}\left(X_{i, r} \times{ }_{Z_{i}} Y_{i, s}\right)$. By Lemma $3, \mathcal{G}\left(X_{i, r} \times Z_{i} Y_{i, s}\right)=\mathcal{G} X_{i, r} \times \mathcal{G} Z_{i} \mathcal{G} Y_{i, s}$. Write $M_{i, r, s}$ for this topological scheme.

By the argument in the last part of the proof of Theorem 1, the schemes, $\mathcal{G}\left(X_{i, r} \times Z_{i} Y_{i, s}\right)$ can be pieced together along their intersections to give $\mathcal{G}\left(X \times_{Z} Y\right)$. On the other hand, the 
shcemes $\mathcal{G} X_{i, r}, \mathcal{G} Z_{i}$ and $\mathcal{G} Y_{i, s}$ piece together to give $\mathcal{G} X, \mathcal{G Z}$ and $\mathcal{G} Y$, respectively, and so their products over the $\mathcal{G} Z_{i}$ piece together to give $\mathcal{G} X \times \mathcal{G} Z \mathcal{G} Y$. Consequently by the local equalities noted just above, $\mathcal{G}\left(X \times{ }_{Z} Y\right)$ and $\mathcal{G} X \times \mathcal{G} Z \mathcal{G} Y$ are isomorphic.

Notice that $X \times_{K} Y$ is abbreviated notation for $X \times_{\operatorname{Spec}(K)} Y$. In the following, we give the basic properties of the Greenberg functor including those properties which show that it is the appropriate object for our consideration.

Proposition 5. Let $X$ be a $K$-scheme of finite type. Then the following hold.

(1) $\mathcal{G}(\operatorname{Spec}(K))=\operatorname{Spec}(k)$.

(2) $\mathfrak{W}_{\pi}(\operatorname{Spec}(k))=\operatorname{Spec} K$.

(3) $\operatorname{Hom}(\operatorname{Spec}(K), X)=\operatorname{Hom}(\operatorname{Spec}(k), \mathcal{G} X)$.

Proof. To prove (1), we apply the definition of a localized Greenberg functor, Definition 14. Let $Y$ be any $k$-scheme. We must show that $\operatorname{Hom}\left(\mathfrak{W}_{\pi}(Y), \operatorname{Spec}(K)\right)=$ $\operatorname{Hom}(Y, \operatorname{Spec}(k))$. But this follows from the fact that $\operatorname{Spec}(K)$ and $\operatorname{Spec}(k)$ are final objects in the categories respectively of local ringed spaces over $K$ and those over $k$. Item (2) is simply a straightforward application of the definition of the functor $\mathcal{G}$. As for item (3), just write $\operatorname{Hom}\left(\mathfrak{W}_{\pi}(\operatorname{Spec}(k)), X\right)=\operatorname{Hom}(\operatorname{Spec}(k), \mathcal{G} X)$ and apply (2).

THEOREM 3. Let $X$ be a group scheme of finite type over $K$. Then $\mathcal{G} X$ is a group in the category of topological schemes over $k$.

Proof. The structure data for $X$ consist of maps, $\mu: X \times_{K} X \rightarrow X, e: \operatorname{Spec}(K) \rightarrow X$ and the inverse map, $s: X \rightarrow X$. Simply apply the functor $\mathcal{G}$ to each of these maps. Since, by Theorem 2, $\mathcal{G}\left(X \times_{K} X\right)=\mathcal{G} X \times_{k} \mathcal{G} X$ these data yield a set of data endowing $\mathcal{G} X$ with the structure of a group in the category of topological schemes.

\section{Spaces of lattices.}

3.1. Lattices of fixed discriminant and height: Let $k$ be the algebraic closure of $\mathbb{F}_{p}$, let $\mathcal{O}=\mathfrak{W}(k)=W_{k}$ be the ring of Witt vectors of $k$ and let $K$ be the fraction field of $\mathcal{O}$. Write $K^{n}$ for the $n$-dimensional $K$-vector space viewed as the space of column vectors of length $n$. Let $F \subset K^{n}$ denote the free rank $n \mathcal{O}$-module spanned by the standard basis. A lattice in $K^{n}$ is a free rank $n \mathcal{O}$-submodule of $K^{n}$; it is called special with respect to $F$ if its $n^{\prime}$ th exterior power is equal to the $n^{\prime}$ th exterior power of $F$. If $\bigwedge^{n} L=p^{q} \bigwedge^{n} F$ then $L$ is of discriminant $p^{q}$ with respect to $F$. We simplify by saying that it is of index $q$. Though index is defined only with respect to $F$ we shall rarely refer to $F$. A further point must be emphasized. In this section we shall be working for the most part with the additive Witt fractions, $Q W_{k}^{+}$. There are a few points which require passage to the perfect completion, $Q W_{k}$, but for the most part we are working with projective schemes and separable limits of them. When we must pass to perfect completions, we shall inform the reader clearly. That these constructions yield separable limits of projective schemes seems to me to be of great importance, though I have not found any particular use for it. In this and in subsequent sections we will write $K$ for $Q W_{k}^{+}=\mathbb{A}_{K}^{1}$. This conflicts with previous practice. The ambiguity of this notation is 
not a problem so long as we work with topological $k$-schemes where $K$ can have little other meaning.

A lattice $M$ in $K^{n}$ will be said to be of height at most $r$ if $p^{-r} F \supseteq M$.

Definition 15. The set of lattices in $K^{n}$ of index $q$ and height at most $r$ will be written $\mathbb{L a t}_{r}^{n, q}(K)$. We shall call it the space of lattices of index $q$ and height at most $r$. Lattices of index 0 will be called the special lattices. We write $\mathbb{L a t}_{r}^{n}(K)$ for $\operatorname{Lat}_{r}^{n, 0}(K)$ and, in argument, where there is no ambiguity, we shall write $\mathbb{L a t}_{r}^{n, q}$ and $\mathbb{L}_{\mathrm{at}}{ }_{r}^{n}$ for these spaces.

In this section we shall show that this space is a projective scheme over $k$; by construction it will be reduced. It will be shown to be irreducible.

Let $L$ be any lattice of height less than $r$. A lattice admits a basis and we may write this as a set of column vectors, $\left(u_{1,1}, \ldots, u_{n, 1}\right)^{T}, \ldots,\left(u_{1, n}, \ldots, u_{n, n}\right)^{T}$. The superscript, $T$, denotes transpose. We may permute these to assume that $u_{1,1}$ is of lowest value among the $u_{1, j}$, then use elementary column operations over the valuation ring to make the other entries in the first row null. Repeat this procedure on the $(n-1) \times(n-1)$-matrix with upper left entry, $u_{2,2}$ and so on. One can obtain a lower triangular matrix $\left(u_{i, j}\right)$ whose columns span the lattice and such that the product, $u_{1,1} u_{2,2}, \ldots, u_{n, n}$, is of value $q$. Further elementary column operations over the valuation ring can be applied to insure that the element $u_{i, j}, j<i$ is either 0 or has value greater than or equal to $-r$ but less than the value of $u_{i, i}$. Since multiplying a basis vector by a unit does not change the lattice we may further assume that the diagonal entries are just powers of $p$. The vector whose entries are the $p$-exponents of the diagonal entries of this matrix will be called the type of the lattice. If $L$ is of type $\left(r_{1}, r_{2}, \ldots, r_{n}\right)$ then the index of $L$ is $\sum_{1}^{n} r_{i}=q$. We shall think of the lattice as the span of these columns, that is as the span of the columns of a lower triangular matrix. For any large enough $s$, the elementary divisors of $L / p^{s} F$ are the integers $p^{s-r_{i}}$ without multiplicity. By the structure theorem for modules over a PID they and their frequencies are uniquely determined subject to the choice of the standard lattice, $F$, and their order is determined by a choice of an ordered basis in $F$. Hence the type of a lattice is uniquely determined. We do not assert this type is orbit type under the Iwahori or the maximal bounded subgroup or that the lower triangular matrix described above is uniquely determined.

Consider the diagonal matrix with entries, $u_{i, i}$ and suppose that it spans a lattice of index, $q$. If $r_{i}$ is the value of $u_{i, i}$, then $r_{i} \geq-r$ and the assumption on index implies that $\sum_{i} r_{i}=q$. These two conditions imply that $r_{i} \leq q+r(n-1)$. This means that if $L$ is of index $q$ and $L \subseteq p^{-r} F$ then $L \supseteq p^{q+r(n-1)} F$. For each $j$, the diagonal matrix with entries, $u_{i, i}=p^{-r}, i \neq j, u_{j, j}=p^{q+r(n-1)}$ meets all the requirements. The intersection of these $n$ lattices of index $q$ is exactly ( $\left.p^{q+(n-1) r}\right) F$ and so no smaller $p$-multiple of $F$ is contained in all the lattices of height at most $r$ and index $q$. That is, the lattices of index, $q$, and height at most $r$ are those lattices $L$ of index, $q$, such that $p^{-r} F \supset L \supset p^{q+(n-1) r} F$.

Definition 16. Let $L$ be a lattice of index $q$. Then a basis of $L$, consisting of vectors $\mathbf{u}_{1}, \ldots, \mathbf{u}_{n}$, so that $\mathbf{u}_{i}=\left(u_{1, i}, \ldots, u_{n, i}\right)^{T}$ and the matrix, $\left(u_{i, j}\right)$ is lower triangular with powers of $p$ on the diagonal will be called a standard basis of $L$. 
LEMMA 7. The group $p^{-r} F / p^{q+(n-1) r} F$ is a unipotent algebraic group of dimension $n q+r n^{2}$ and the lattices of rank $n$, height at most $r$ and index $q$ are in bijective correspondence with its $k^{*}$-stable connected subgroups of dimension $(n-1)(q+n r)$. Here $k^{*}$ is assumed to act through the multiplicative representative morphism, $\xi: k^{*} \rightarrow \mathcal{O}^{*}$.

PROOF. It is clear that $p^{-r} F / p^{q+r(n-1)} F$ is a finite dimensional commutative unipotent linear algebraic group over $k$ admitting in addition an $\mathcal{O}$-structure. Then by means of $\xi$ the multiplicative group acts on $p^{-r} F / p^{q+r(n-1)} F$ and a subvariety of it is an $\mathcal{O}$-submodule if and only if it is a subgroup and hence stable under multiplication by $p$ and also $k^{*}$-stable. This is so because it is then stable under sums, $\sum_{i} \xi\left(t_{i}\right)^{p^{-i}} p^{i}$ and so under all of $\mathcal{O}$. Finally, notice that $M$ is a lattice of height at most $r$ and index $q$ if and only if the length of $p^{-r} F / M$ is $q+n r$ and that this length is the same as its dimension as an algebraic group. Since the codimension of $M$ in $p^{-r} F$ is $n r+q$, the dimension of $M / p^{q+r(n-1)} F$ is $(n-1)(q+n r)$, that is, $n(q+n r)$, the dimension of $p^{-r} F / p^{q+(n-1) r} F$ less the codimension of $M$ in $p^{-r} F$. Further, for any non-zero element of $p^{-r} F / p^{q+(n-1) r} F$, the closure of its $k^{*}$-orbit contains 0 . Thus the set of lattices of index $q$ and height at most $r$ is in bijective correspondence with the set of $k^{*}$-stable connected algebraic subgroups of $p^{-r} F / p^{q+r(n-1)} F$ of dimension $(n-1)(q+n r)$.

We introduce a notion which is of great utility in the following discussion. Let $X$ be a $k$-scheme and let $q: U \rightarrow X$ be an $X$-scheme with a section, $e: X \rightarrow U$. Then we shall say that $U$ is flat over $X$ off $e$ if $U \backslash e(X)$ is flat over $X$.

LEMMA 8. Let $X$ be a $k$-scheme and let $N$ be a connected reduced linear algebraic group over $k$. Let $U \subseteq X \times_{k} N, \phi: U \rightarrow X$ be a family of closed subschemes of $N$ containing the image of the identity section, $e: X \rightarrow N$ and with fibers stable under taking inverses. Assume that $N$ is faithfully flat off $e$. Then there is a unique maximal closed reduced subscheme of $X$, denoted $Y$, so that if $g: Z \rightarrow X$ is any map such that $Z \times_{X} U$ is a $Z$ subgroup scheme of $Z \times_{k} N$ then $\bar{g}(Z)_{\text {red }} \subseteq Y$.

Proof. Let $U^{\prime}=U \backslash e(X)$. Let $\bar{m}: U^{\prime} \times{ }_{X} U^{\prime} \rightarrow X \times \times_{k} N$ be the restriction of the group multiplication. Then, $T=\left(X \times_{k} N\right) \backslash U$ is open and thus so also is $\bar{m}^{-1}(T)$. But $\phi \mid U^{\prime}$ is flat and hence open and hence $\phi\left(\bar{m}^{-1}(T)\right)$ is open. Take $Y=X \backslash \phi\left(\bar{m}^{-1}(T)\right)$, the complement of $\phi\left(\bar{m}^{-1}(T)\right)$. It is closed and by definition it consists of the set of points $x$, so that if $u_{1}, u_{2} \in \phi^{-1}(x)=U_{x}$ then $m^{\prime}\left(u_{1}, u_{2}\right) \in U$. Let $U_{Y}=\phi^{-1}(Y), U_{Y}^{\prime}=U_{Y} \backslash e(Y)$. It follows that $m^{\prime}\left(U_{Y}^{\prime} \times_{Y} U_{Y}^{\prime}\right) \subseteq U_{Y}$ and since the latter is closed and $U_{Y} \times_{Y} U_{Y}$ is in the closure of $U_{Y}^{\prime} \times{ }_{Y} U_{Y}^{\prime}$, multiplication carries it into $U_{Y}$. By hypothesis the fibers of $U_{Y}$ are stable under taking inverses and it admits an identity section. Examination shows $Y$ to be exactly the set of points with $U$ fibers which are subgroups.

PROPOSITION 6. Let $q, m$ and $N$ be three integers such that $q<m$ and $N<n(m-q)$. Then there is a $k$-scheme $\mathbb{L}(q, m ; N)$ which is projective and of finite type over $k$ and a flat commutative group scheme, $\mathbb{U}(q, m ; N) \subseteq p^{q} F / p^{m} F \times_{k} \mathbb{L}(q, m ; N)$ which is a universal family of flat subschemes of $p^{q} F / p^{m} F$ of dimension, $N$, parametrized by $\mathbb{L}(q, m ; N)$. That 
is, for any $k$-scheme, $Y$, and any flat $Y$-group scheme, $\mathcal{M} \subseteq p^{q} F / p^{m} F \times_{k} Y$, whose fibers are $\mathcal{O}$-submodules of $p^{q} F / p^{m} F$ of dimension $N$, there is a unique map, $f: Y \rightarrow \mathbb{L}(q, m ; N)$ so that $\mathcal{M}=Y \times_{\mathbb{L}(q, m ; N)} \mathbb{U}(q, m ; N)$.

Proof. We must consider $p^{q} F / p^{m} F$ as a $k^{*}$-variety. An element of this group can be thought of as a column vector with entries, $u_{1}, \ldots, u_{n}$ where $u_{i}$ is an element of $p^{q} \mathcal{O} / p^{m} \mathcal{O}$. Hence we may write $u_{i}$ as a sum, $u_{i}=\sum_{j=q}^{m-1} \xi\left(x_{i, j}\right)^{p^{-j}} p^{j}$. Now by (2.2), $\xi(t) u_{i}$ has $i, j$ coordinate $t^{p^{j}} x_{i, j}$. This has the following consequence. Let $L=p^{q} F / p^{m} F$ and let $L^{\prime}=L \backslash 0$. Then $L^{\prime} / k^{*}$ is the weighted projective space $\operatorname{Proj}\left(k\left[x_{1, q}, \ldots, x_{n, m-1}\right]\right)$ where the polynomial ring is graded by taking $x_{i, j}$ of degree $p^{j}$ (even when $j$ is negative). We shall call this space $\mathbb{P L}_{n}^{q, m}$. Notice that the $k^{*}$-stable closed subschemes of $L$ of dimension $N$ are functorially in bijective correspondence with closed $N-1$ dimensional subschemes of $\mathbb{P L}_{n}^{q, m}$ which is projective and of finite type over $k$. Notice that the projective coordinate ring of $\mathbb{P L}_{n}^{q, m}$ is graded by $p^{q} \mathbb{Z}^{n}$.

Let $H$ denote the Hilbert scheme of closed subschemes of $\mathbb{P L}_{n}^{q, m}$ of dimension $N-1$. Let $\overline{\mathcal{U}}$ in $\mathbb{P L}_{n}^{q, m} \times_{k} H$ denote the universal family. Let $\mathcal{U}^{\prime}$ denote the inverse image of $\overline{\mathcal{U}}$ in $L^{\prime} \times_{k}$ $H$. Let $\mathcal{U}^{\prime \prime}$ denote the closure of $\mathcal{U}^{\prime}$ in $L_{r} \times_{k} H$ and let $e_{H}: H \rightarrow \mathcal{U}^{\prime \prime}$ denote the 0 -section. This is a family of subschemes of $L$ of dimension $N$ faithfully flat off $e_{H}$. We note that this is exactly the family of closed $k^{*}$-stable subschemes of $L$ of dimension $N$. The graded ring, $k\left[x_{1, q}, \ldots, x_{n, m-1}\right]$ is graded by strictly positive degrees. This means that in its spectrum, 0 is the unique fixed point and it is in the closure of every $k^{*}$-orbit. Hence, since the fibers of $\mathcal{U}^{\prime \prime}$ are $k^{*}$-stable and closed, they are connected. The hypotheses of Lemma 8 exactly apply to $\mathcal{U}^{\prime \prime}$ as a subscheme of $H \times{ }_{k} L_{r}$. Hence there is a closed reduced subscheme of $H$ whose points are exactly those whose $\mathcal{U}^{\prime \prime}$ fibers are closed $k^{*}$-stable subgroups of $p^{q} F / p^{m} F=L$. Denote this subscheme $Z$, write $\mathcal{U}_{Z}$ for the restriction of $\mathcal{U}^{\prime \prime}$ to $Z$ and write $e_{Z}$ for the restriction of the zero section to $Z$. It is a group subscheme of $Z \times_{k} p^{q} F / p^{m} F$ faithfully flat off $e_{Z}$.

Consider $\mathcal{U}_{Z}$. If $a$ is a non-null point in the $\mathcal{U}_{Z}$ fiber over $z$, there is an fpqc neighborhood of $z, T$ and a section $s: T \rightarrow \mathcal{U}_{Z} \times{ }_{Z} T=\mathcal{U}_{T}$ whose value on points over $z$ is $a$ and which is nowhere zero on $T$. Translation by $s$ is an automorphism of $\mathcal{U}_{T}$ carrying the zero section to $s$ and since $\mathcal{U}_{T}$ is flat off $e_{T}$ it is flat at $s$. Since a neighborhood of $s$ is, by translation, isomorphic to a neighborhood of $e_{T}$, it is flat at the zero section as well. Hence $\mathcal{U}_{T}$ is flat over $T$. It follows that on components where $\mathcal{U}_{Z}$ is not null, it is flat.

Now suppose $Y$ is a $k$-scheme and that $\mathcal{M} \subseteq p^{q} F / p^{m} F \times_{k} Y$ is a flat family of $\mathcal{O}$ submodules of dimension $N$. Then one may take the complement of the null section in $\mathcal{M}$ and take its quotient to obtain a flat family of $N-1$ subschemes of $\mathbb{P L}_{n}^{q, m}$. This determines a map, $f: Y \rightarrow H$. Since $M$ is a family of $\mathcal{O}$-modules, $f(Y) \subseteq Z$ and $M$ is the pull back of $\mathcal{U}_{Z}$. That is, $Z$ is the scheme, $\mathbb{L}(q, m ; N)$ and the universal family, $\mathcal{U}_{Z}$ can be taken to be the group scheme, $\mathbb{U}(q, m ; N)$.

PROPOSITION 7. Let $\mathbf{u}_{1}, \ldots, \mathbf{u}_{n}$ be a set of columns which is a standard basis of height at most $r$, index $q$ and type $\left(r_{1}, \ldots, r_{n}\right)$. Let $e_{i}$ denote the $i^{\prime}$ th standard basis vector. Then, we have the following: 
(1) Suppose $r_{i}>r_{i+1}$. There is a flat family of lattices of height at most $r$ and index $q$ parametrized by $\mathbb{A}_{k}^{1}$ of type $\left(r_{1}, \ldots, r_{i-1}, r_{i+1}, r_{i}, \ldots, r_{n}\right)$ for all values of t except 0 where it is the lattice spanned by $\mathbf{u}_{1}, \ldots, \mathbf{u}_{n}$.

(2) Iffor some $i<n, \boldsymbol{u}_{i}=p^{r_{i}} e_{i}$ and $r_{i}>-r$, there is a flat family of lattices of height at most $r$ and index $q$ parametrized by $\mathbb{A}_{k}^{1}$ which consists of lattices of type $\left(r_{1}, \ldots, r_{i}-\right.$ $\left.1, \ldots, r_{n}+1\right)$ except at 0 where it is the span of $\mathbf{u}_{1}, \ldots, \mathbf{u}_{n}$.

ProOF. Let $Y$ be a $k$-scheme and suppose that $s_{i}: Y \rightarrow p^{-r} F, i=1, \ldots, n$ is a set of maps so that for each closed point, $y \in Y$, the values $s_{i}(y)$ are a basis of a lattice of height at most $r$ and index $q$. Let $\mathbb{L}=\mathbb{L}\left(-r, q+r(n-1), n q+r n(n-1)-\sum_{i} r_{i}\right)$ and let $N=$ $n q+r n(n-1)-\sum r_{i}$. Then for each $i, s_{i} \times$ id is a map from $Y \times{ }_{k} \mathbb{L}$ to $p^{-r} F / p^{(n-1) r+q} F \times_{k} \mathbb{L}$ and $\left(s_{i} \times \mathrm{id}\right)^{-1}(Y \times \mathbb{U}(-r, q+r(n-1), N))=T_{i}$ is closed in $Y \times_{k} \mathbb{L}$ and its closed points are pairs $(y, L)$ where $L$ is a lattice containing $s_{i}(y)$. Let $\Gamma=T_{1} \cap \cdots \cap T_{n}$. Then the closed points of this set are the pairs $(y, L)$ such that $\left\{s_{1}(y), \ldots, s_{n}(y)\right\} \subseteq L$. Since the vectors $s_{i}(y)$ constitute a basis, $L$ is uniquely determined. That is, $\Gamma \cap y \times \mathbb{L}$ is a single point. Hence $\Gamma$ is the graph of a morphism $f: Y \rightarrow \mathbb{L}$. It is clear that $f^{-1}(\mathbb{U}(-r, q+r(n-1), N))$ is a flat family whose fiber at $y$ is the span of $s_{1}(y), \ldots, s_{n}(y)$.

Let $\xi$ denote the multiplicative representative map. To establish (1), let $s_{j}(t)=\mathbf{u}_{j}$ for each $j \neq i+1$. Let $s_{i+1}(t)=\mathbf{u}_{i+1}+\xi(t) p^{r_{i+1}} e_{i}$. At 0 this gives the specified lattice. At $t=c \neq 0$ the specified base is not standard. However $\mathbf{u}_{1}, \ldots, \mathbf{u}_{i-1}, s_{i+1}(c), s_{i}-$

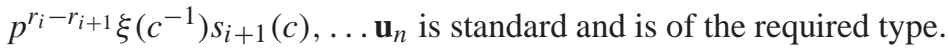

To establish (2) let $s_{j}(t)=\mathbf{u}_{j}, j \neq n$ and let $s_{n}(t)=p^{r_{n}} e_{n}+\xi(t) p^{r_{i}-1} e_{i}$. The values of these maps give a basis for a lattice of index $q$ for all $t$ and so they give a flat family by the procedure of the first paragraph. When $t \neq 0$ the base is not standard. However $s_{i}-\xi\left(t^{-1}\right) p s_{n}=\xi\left(t^{-1}\right) p^{r_{i}+1}$. Permute this vector with $s_{n}$ and the result is a base in standard form and of the right type.

THEOREM 4. For each $q>-n r$, there is a reduced and irreducible $k$-scheme projective and of finite type over $k$, denoted $\mathbb{L a t}_{r}^{n, q}$, and a universal family of flat group subschemes of $\mathbb{L a t}_{r}^{n, q} \times_{k}\left(p^{-r} F / p^{r(n-1)+q} F\right)$ parametrized by $\mathbb{L a t}_{r}^{n, q}$. We denote it $\mathbb{U}_{r}^{n, q}(K)$ or, when there is no ambiguity, $\mathbb{U}_{r}^{n, q}$. If $Y$ is any reduced $k$-scheme and $M \subseteq Y \times{ }_{k}\left(p^{-r} F / p^{r(n-1)+q} F\right)$ is any flat group subscheme of lattices of index $q$ and height at most $r$, then there is a unique morphism $f: Y \rightarrow \mathbb{L a t a t}_{r}^{n, q}$ so that $M \simeq Y \times_{\mathbb{L a t}_{r}^{n, q}} \mathbb{U}_{r}^{n}$.

Proof. Let $F_{q}$ denote the lattice with basis, $p^{-r} e_{1}, \ldots, p^{-r} e_{n-1}, p^{q+(n-1) r} e_{n}$. Let $Z=\mathbb{L}(-r,(n-1) r+q ;(n-1)(q+n r))$, the scheme given by Proposition 7 , and let $\mathcal{U}_{Z}=\mathbb{U}(-r,(n-1) r+q ;(n-1)(q+n r))$. Now $Z$ is a disjoint union of components, and on each the Hilbert polynomial of its $k^{*}$-quotient is constant. Since the degree of a Hilbert polynomial corresponds to the dimension of the subscheme, the points of $Z$ corresponding to $k^{*}$-stable subgroup schemes of dimension $N$ are a union of connected components. Now consider the component of this scheme containing the point corresponding to $F_{q} / p^{(n-1) r+q} F$. 
We wish to prove that this component posesses all the properties claimed for $\mathbb{L a t}_{r}^{n, q}$. Call this component $X$ and write $\mathcal{U}$ for the restriction of $\mathcal{U}_{Z}$ to $X$.

First we must show that if $M$ is any lattice of index $q$ and height at most $r$, it is in the same connected component as $F_{q}$. Suppose that $e_{i}$ is the $i$ 'th vector in the standard basis and let $p^{r_{1}} e_{1}, \ldots, p^{r_{n}} e_{n}$ span a lattice of index $q$ and height at most $r$. Suppose that $r_{1}=r_{2}=$ $\cdots=r_{i-1}=-r, r_{i}>-r$. Then we may construct a degeneration of the type specified by (2) of Proposition 7. The proposition implies that the given lattice is in the closure of the set of lattices of type $\left(-r, \ldots,-r, r_{i}-1, \ldots, r_{n}+1\right)$. The existence of this degeneration implies that the two types are in the same component of the Hilbert scheme we are considering. We may repeat this construction repeatedly until we have demonstrated that every lattice of index $q$ and height at most $r$ is connected by a sequence of degenerations to a lattice of type $(-r, \ldots,-r,(n-1) r+q)$. Moreover Proposition 7 implies that each lattice of index $q$ and height at most $r$ is in the closure of the set of lattices of type $(-r, \ldots,-r,(n-1) r+q)$. Similarly $F_{q}$ is in the same component by definition. This means that all lattices of index $q$ and height at most $r$ are in the same component of the Hilbert scheme and that this must be $X$.

Now observe that the group $G=S L\left(n, \mathcal{O} / p^{r n+q} \mathcal{O}\right)$ operates on $X$. Up to a Frobenius twist, this is an algebraic action. The orbit of the lattice of type $(-r, \ldots,-r,(n-1) r+q)$ is stable under these automorphisms and so its closure, which we will write $X_{0}$, is as well. Hence this closure contains all the $G$ translates of any element in it. Now $G$ is a quotient of a maximal bounded subgroup of $S L(n, K)$, and so it contains the image of an Iwahori subgroup. Denote this image $B$. The scheme, $X$, is stable under the action of $S L(n, \mathcal{O})$ and hence of its Iwahori subgroups and the kernel of the natural surjection of $S L(n, \mathcal{O})$ acts trivially on $X$. Hence the $G$ - and $B$-orbits in $X$ are actually the orbits of, respectively, a maximal bounded subgroup and an Iwahori subgroup. By the Iwahori decomposition (actually the Birkhoff decomposition, see Subsection 3.4 below) it is a union of $B$-orbits of elements, $\gamma=\exp _{p}\left(r_{1}, \ldots, r_{n}\right)$. Here, $\exp _{p}\left(r_{1}, \ldots r_{n}\right)$ denotes the lattice spanned by the columns of a diagonal matrix with $i$ 'th diagonal entry, $p^{r_{i}}$. (See Subsection 3.4 below.) Since this closure contains elements of any type of index $q$ and height at most $r$ by the remarks above, it contains all lattices of index $q$ and height at most $r$. That is, all the lattices of index $q$ and height less than $r$ are contained in the closure of a reduced and irreducible open subset. This establishes the irreducibility of $X$.

Let $U_{x}$ be the fiber of $\mathcal{U}$ over $x \in X$. It is a unipotent group subscheme of $p^{-r} F /$ $p^{(n-1) r+q} F$ of dimension $n r$. Consequently the connected component of its reduced subscheme is likewise. Hence this reduced connected subgroup of dimension $n(n-1) r$, which we denote $U_{0}$, is a lattice of index $q$ and height at most $r$ and so corresponds to a point of $X$. Hence its Hilbert polynomial is the Hilbert polynomial of $F_{q} / p^{(n-1) r+q} F$ and so also of $U$. But the Hilbert polynomial of $U$ cannot be equal to the Hilbert polynomial of $U_{0}$ unless $U=U_{0}$. Thus $U=U_{0}$. That is the fibers of $\mathcal{U}$ are connected, non-singular and $k^{*}$-stable. That is, they are lattices, special and of height at most $r$.

Suppose that $Y$ is a $k$-scheme and that $\mathcal{N} \subseteq Y \times{ }_{k} L_{r}$ is a flat family of special sublattices. Then it is $k^{*}$-stable. Further $\mathcal{N} \backslash e(Y)$ is flat and $k^{*}$-stable whence the quotient, $(\mathcal{N} \backslash e(Y)) / k^{*}$ 
is also. This determines a map into $H$ and Lemma 8 assures that its image lies in $Z$ and hence, by the connectedness argument above, in $X$. It is of no great depth to ascertain that $\mathcal{N}$ is isomorphic to $\mathcal{U} \times_{X} Y$. That is, $X=\mathbb{L a t}_{r}^{n, q}$ and $\mathcal{U}$ is its universal family, $\mathbb{U}_{r}^{n, q}(K)$.

REMARK 2. We shall always write $\mathbb{L a t}_{r}^{n}(K)$ for $\mathbb{L a t}_{r}^{n, 0}(K)$. One may construct morphisms of lattice varieties corresponding to multiplication by $p$ or by arbitrary elements of $S L(n, K)$. They cannot be constructed by simply applying the action as this requires passing to complete perfect topological schemes. They can be constructed by other strategies. This problem is related to the question of representing the Steinberg group as a topological scheme and it is the topic of a work in preparation. Using these morphisms lattice schemes of arbitrary index can be shown to be isomorphic to subschemes of schemes $\mathbb{L a t}_{r}^{n, q} 0 \leq q<n$.

COROLlaRY 1. Let $X_{r, n}=\mathbb{L a t}_{r}^{n, q}(K)$. Let $M \subseteq \mathbb{U}_{r}^{n, q}(K)$ be a closed $k^{*}$-stable reduced group subscheme of $\mathbb{U}_{r}^{n}(K)$ and let $\phi: M \rightarrow X_{r, n}$ be the structure morphism. Let $X_{s}(M)=\left\{x: \operatorname{dim}\left(\phi^{-1}(x)\right) \geq s\right\}$. Then for each $s, X_{s}(M)$ is a closed subset of $X_{r, n}$.

PROOF. First replace $M$ by the reduced subscheme of one of its irreducible components. If the assertion is true for each of these components, then $X_{S}(M)$ is the union of the sets corresponding to each of the reduced subsets of irreducible components and so if they are closed the finite union is also. Hence we may assume that $M$ is reduced and irreducible. Hence $M$ is integral and so $M_{s}=\left\{x \in M: \operatorname{dim}\left(\phi^{-1}(\phi(x))\right) \geq s\right\}$ is a closed subset of $M$ by upper semicontinuity of fiber dimension. Moreover $M_{s}$ is a union of fibers of $\phi$ and so $k^{*}$-stable.

Let $Z$ be the zero section in $\mathbb{U}_{r}^{n}(K)$. It is evident that $M_{s} \cap\left(\mathbb{U}_{n}^{r}(K) \backslash Z\right)$ is a closed $k^{*}$ stable subset of the cone over $\mathbb{P} \mathbb{L}_{n}^{r}$. That is it is the cone over a closed subset of $\mathbb{P} \mathbb{L}_{n}^{r} \times_{k} X_{r, n}$. By properness, it follows that its image in $X_{r, n}$ is closed. This image is exactly $X_{s}(M)$.

By construction $\mathbb{L a t}_{r}^{n, q}$ is a finite dimensional scheme. Observe that the subgroup of $S L(n, \mathcal{O})$ consisting of those matrices congruent to one $\bmod p^{n r+q}$ acts trivially on $\mathbb{L a t}_{r}^{n, q}$. Hence the quotient group, which is finite dimensional, operates on this space and it does so, by the argument in the proof above, with a finite number of orbits.

There is a natural inclusion, $j_{r, s}^{q}: \mathbb{L a t}_{r}^{n, q}(K) \rightarrow \mathbb{L a t}_{s}^{n, q}(K)$. This sequences of varieties and inclusions is a direct system. Consequently, the $\operatorname{limit}_{\lim _{r}} \mathbb{L a t}_{r}^{n, q}(K)$ is a topological scheme.

DEFinition 17. The direct limit, $\lim _{r} \mathbb{L a t}_{r}^{n, q}(K)$ is called the space of lattices of rank $n$ and index $q$ in $K$. It is written $\mathbb{L a t}{ }^{n, q}(K)$. We write $\mathcal{U}_{K}^{n, q}$ for the universal family of lattices of index, $q$. We shall write $\widetilde{\mathbb{L a t}}^{n, q}(K)$ and $\tilde{\mathcal{U}}_{K}^{n, q}$ for the corresponding perfect completions. For $q=0$ we write $\mathbb{L a t}^{n}(K)$ and refer to it as the space of special lattices and the same for the universal family mutatis mutandis.

We conclude this section with some remarks on the action of $S L(n, K)$ on $\mathbb{L a t}^{n}(K)$ and its perfect completion. 
DEFINITION 18. Let $X$ be a topological scheme over $k$. We shall call $X$ pseudohomogeneous if there is a group of automorphisms transitive on the closed points of $X$. If $G$ is a group and $G$ acts transitively on closed points, we shall say that $X$ is pseudohomogeneous over $G$.

It can be proven that the closed points of $S L(n, K)$ operate on $\widetilde{L}^{n}{ }^{n}(K)$ transitively (see Remark 2). However there is no algebraic action of $S L(n, K)$ on $\widetilde{\mathbb{L a t}}^{n}(K)$. If the action of $S L(n, K)$ were somehow algebraic its restriction to the maximal torus would be as well. Let $\Delta\left(a_{1}, \ldots, a_{n}\right)$ denote a diagonal matrix with diagonal entries, $a_{i}$ and let $T=\left\{\Delta\left(a_{1}, \ldots, a_{n}\right)\right.$ : $\left.\prod_{1}^{n} a_{i}=1\right\}$ be a maximal torus. Then the map which sends an element of $T, \Delta\left(a_{1}, \ldots, a_{n}\right)$ to the lattice spanned by the vectors, $a_{i} e_{i}$ is the orbit map for the action of $T$ on the standard lattice, $F$. The image of $T$ however is a discrete set. Hence this action under which $\widetilde{L a t}^{n}(K)$ is pseudohomogeneous is not algebraic. On the other hand $S L(n, \mathcal{O})$ acts on $F$ and so on its multiples, $p^{-r} F$ and $p^{(n-1) r} F$, and so on their quotient and on the set of $k^{*}$-stable subgroups of fixed codimension. That is, $S L(n, \mathcal{O})$, up to a suitable Frobenius twist, operates algebraically on $\mathbb{L a t}_{r}^{n}(K)$. The subgroup $\Gamma_{r}=\left\{\gamma \in S L(n, \mathcal{O}): \gamma \equiv \mathrm{id} \bmod p^{n r}\right\}$ operates trivially and so the finite dimensional group, $S L(n, \mathcal{O})^{r} / \Gamma_{r}=S L\left(n, \mathcal{O} / p^{n r} \mathcal{O}\right)^{(r)}$ acts on $\mathbb{L a t}_{r}^{n}(K)$. (Recall the upper $(r)$ signifies a Frobenius cover.) Moreover these actions, up to the Frobenius twist, are coherent.

3.2. General lattices: We have not constructed a space of ordinary bounded lattices. In this section we do so. First, we introduce the convention, $\bigwedge^{-n} L=\left(\bigwedge^{n} F: \bigwedge^{n} L\right) \bigwedge^{n} F$ where $(N: M)=\{x \in \mathcal{O}: x M \subseteq N\}$. Our construction is based on the following observation. If $L \subseteq K^{n}$ is a general lattice, and $F \subseteq K^{n}$ is a standard lattice, then $L \oplus \bigwedge^{-n} L$ is a special lattice in $K^{n} \oplus \bigwedge^{n} K^{n}$ with respect to the distinguished lattice, $F \oplus \bigwedge^{n} F$.

Write $M$ for the $(n+1)$-dimensional vector space, $K^{n} \oplus \bigwedge^{n}\left(K^{n}\right)$ and $\tilde{F}$ for the lattice $F \oplus \bigwedge^{n} F$ in $M$. A lattice $L$ in $M$ will be said to be $\Lambda$-decomposable if $L=\left(L \cap K^{n}\right) \oplus$ $\left(L \cap \bigwedge^{n} K^{n}\right)$. Let $e_{1}$ be the projection of $M$ onto $K^{n}$ and let $e_{2}$ be the projection of $M$ on $\bigwedge^{n}\left(K^{n}\right)$. Then $e_{1}$ and $e_{2}$ are orthogonal idempotents and, since $e_{j}=\mathrm{id}-e_{i}$, the lattice $L$ is $\Lambda$-decomposable if and only if $e_{i} L \subseteq L$ for either one of the two $e_{i}$. Let $F_{r, s}=p^{-r} F \oplus$ $p^{-s} \wedge^{n} F$. It is nearly tautological to note that any of the lattices, $F_{r, s}$, are $\Lambda$-decomposable, that is to say $e_{i}$-stable for each $r, s$. In particular $e_{1}$ and $e_{2}$ induce corresponding projections, $\bar{e}_{i}$ on $F_{r, s} / F_{a, b}$ for any $a \leq r, b \leq s$.

Let $L \subseteq K^{n}$ be a lattice. We shall always write $\tilde{L}$ for $L \oplus \bigwedge^{-n} L$. Then $L$ is of index at most $s$ and height at most $r$ if and only if $p^{n r} F_{1} \subseteq \tilde{L} \subseteq F_{r, s}$. If $L$ is of index at most $s$ and height at most $r$ then $p^{n r+s} \tilde{F} \subseteq \tilde{L} \subseteq F_{r, s}$. Then $\operatorname{dim}\left(\tilde{L} / p^{n r+s} \tilde{F}\right)=(n+1)(n r+s)$.

PROPOSITION 8. For each $s \leq r$, there is a unique closed subscheme $Z_{r, s}^{n} \subseteq \mathbb{L a t}_{r}^{n+1}(K)$ consisting of the $\Lambda$-decomposable lattices of index at most $s$ and height at most $r$. Further if $\mathbb{E}_{r, s}^{n}=\mathbb{U}_{r}^{n+1}(K) \mid Z_{r, s}^{n}$, the scheme of lattices, $\mathbb{E}_{r, s}^{n}$ decomposes into a direct sum of the two lattices, $\bar{e}_{i} \mathbb{E}_{r, s}^{n}$ and if $X$ is any scheme with a flat family of special lattices, $\mathbb{H}$, of rank $n+1$ which is a sublattice of $X \times F_{r, s}$ and each of which is $\Lambda$-decomposable, there is a unique map, $f: X \rightarrow Z_{r, s}^{n}$ so that $\mathbb{H}=\mathbb{E}_{r, s}^{n} \times Z_{r}^{n} X$. 
PROOF. Consider $\mathbb{L a t}_{r}^{n+1}$. Consider the intersection, $\left(F_{r, s} \times \mathbb{L} a t_{r}^{n+1}\right) \cap \mathbb{U}_{r}^{n+1}(K)$. This is a closed $k^{*}$-stable subset of the universal family and so its reduced image in $\mathbb{L a t}_{r}^{n+1}$ is a closed subscheme which we denote $T$.

Let us consider the maps $\bar{e}_{i}$ on $T \times F_{r, s} / p^{n r+s} \tilde{F}$. Write $\bar{F}$ for this group scheme over $T$ Write $\mathbb{U}_{r, s}^{n}(K)$ for the restriction of the universal family to $T$ and write $\overline{\mathbb{U}}$ for its image in $\bar{F}$. Then $\bar{e}_{i}(\overline{\mathbb{U}})=\operatorname{ker}\left(\bar{e}_{j}\right)(i \neq j)$ because the $\bar{e}_{i}$ are orthogonal idempotents. Hence $\bar{e}_{i}\left(\mathbb{U}_{r, s}^{n}(K)\right.$ is closed and so $\bar{e}_{i}\left(\mathbb{U}_{r, s}^{n}(K)\right) \cap \mathbb{U}_{r, s}^{n}(K)$ is also. Moreover it is $k^{*}$-stable in $\mathbb{U}_{r, s}^{n}(K)$. Thus $\left(\bar{e}_{1}\left(\mathbb{U}_{r, s}^{n}(K)\right) \cap \mathbb{U}_{r, s}^{n}(K)\right) \times_{T}\left(\bar{e}_{2}\left(\mathbb{U}_{r, s}^{n}(K)\right) \cap \mathbb{U}_{r, s}^{n}(K)\right)$ is closed in $\mathbb{U}_{r, s}^{n}(K) \times_{T} \mathbb{U}_{r, s}^{n}(K)$. The maximal fiber dimension of this group scheme is $(n+1)(s+n r)$. The set of points at which this fiber dimension is achieved is closed and $k^{*}$-stable in $\mathbb{U}_{r, s}^{n}(K) \times_{T} \mathbb{U}_{r, s}^{n}(K)$. By Corollary 1 above this defines a closed subscheme of $T$. This is readily seen to be the subscheme of $\Lambda$-decomposable lattices and $\mathbb{E}_{r, s}^{n}$ is simply the restriction of the universal family to it. Since the $\bar{e}_{i}$ induce a decomposition, $\mathbb{E}_{r, S}^{n}$ itself decomposes globally.

Definition 19. Write $\mathbb{G L a t}_{r, s}^{n}(K)$ for the scheme of $\Lambda$-decomposable lattices of index at most $s$ and height at most $r$ in $\mathbb{L a t}_{r}^{n+1}(K)$ and write $\mathbb{G} \mathbb{U}_{r, s}^{n}$ for the scheme of lattices, $\left(e_{1} \times \mathrm{id}\right)\left(\mathbb{E}_{r}^{n}\right)$. Then $\mathbb{G U}_{r, s}^{n}$ will be called the universal lattice of rank $n$ index at most $s$ and height at most $r$.

THEOREM 5. $\mathbb{G L a t}_{r, s}^{n}(K)$ is a disjoint union of connected irreducible components denoted $\mathbb{G L a t}_{r, s}^{n, q}(K), s \geq q \geq n r$. Let $\mathbb{G} \mathbb{U}_{r, s}^{n, q}$ denote the restriction of $\mathbb{G}_{r, s}^{n}$ to $\mathbb{G L a t}_{r, s}^{n, q}(K)$. Then the fibers of $\mathbb{G}_{r, s}^{n, q}$ are the lattices of index $q$ and height at most $r$.

Proof. Write $Z_{n, r}=\mathbb{G L}_{\mathrm{Lat}}^{n}(K)$. Write $E=\mathbb{E}_{r}^{n} / p^{n r} F_{1}$. In the notation of the proof of Proposition $8, E=\bar{e}_{1}(E) \times Z_{n, r} \bar{e}_{2}(E)$. Now $E$ is finite dimensional and flat over $Z_{n, r}$ and each factor admits a section and so each of its factors must also be flat. Now the fibers of a flat morphism are equidimensional on connected components.

If the fiber of $\bar{e}_{1}(E)$ at $z$ is of index $q$, then that fiber is of dimension, $(n-1)(n r+q)$. Since $\bar{e}_{1}(E)$ is flat, the index is constant on connected components. On the other hand, Proposition 7 implies that if $L$ is of height at most $r$ and of type $\left(r_{1}, \ldots, r_{n}\right)$ and index $q$, it can be connected by a series of degenerations to the diagonal lattice of type, $(-r, \ldots,-r,(n-1) r+q)$ in such a way that all are in the closure of the set of lattices of this type. Since, by hypothesis, $q \leq s \leq r$ this diagonal lattice is of index $q$ and in $\mathbb{G L a t}_{r, s}^{n}(K)$. Hence the lattices of index, $q$, lie in one irreducible component. Consequently the set of points where the fiber of $\mathbb{G} \mathbb{U}_{r, s}^{n, q}(K)$ is of index $q$ constitute an irreducible component. The rest of the theorem follows.

It is now clear that, for $r \geq q$, the rank $n$ lattices of index $q$ and height at most $r$ are a subscheme of the lattices of index $q$ and height at most $r+1$ and $G \mathbb{U}_{r+1, s}^{n, q}$, the universal family, restricts to $\mathbb{G} \mathbb{U}_{r, s}^{n, q}$.

Definition 20. For $q \leq r$ write $\mathbb{G L a t}_{r}^{n, q}(K)$ for the scheme, $\mathbb{G} \mathbb{L} a t_{r, r}^{n, q}(K)$ and $\mathbb{U}_{r}^{n, q}$ for $\mathbb{U}_{r, r}^{n, q}$. Write $\mathbb{G L a t}_{q}^{n}(K)$ for the limit of the schemes, $\mathbb{G} \mathbb{L a t}_{r}^{n, q}(K), \quad r \geq q$, and write 
$\mathbb{G} \mathbb{U}_{q}^{n}(K)$ for the limit of the schemes, $\mathbb{G} \mathbb{U}_{r}^{n, q}$. We shall refer to these as the scheme of general lattices of rank, $n$, and index $q$ and the universal general rank $n$ index $q$ lattice, respectively. Write $\widetilde{\mathbb{G L a t}}_{q}^{n}(K)$ and $\widetilde{\mathbb{G U}}_{q}^{n}$ for their perfect completions.

3.3. Lattice classes: Consider the lattices of rank $n$ in $K^{n}$. Declare two of them, $L$ and $L^{\prime}$ to be equivalent if $L^{\prime}=\lambda L$ for some non zero constant, $\lambda \in K^{*}$. The resulting equivalence classes will be called additive lattice classes. It is self-evident that $G L(n, K)$ operates transitively on the set of additive lattice classes, that $K^{*}$ operates trivially and that the stabilizer of the standard additive lattice class is $K^{*} \cdot G L(n, \mathcal{O})$. Thus the additive lattice classes are at least set-theoretically a homogeneous space for the topological $k$-group scheme, $P G L(n, K)$. We will show that, like special lattices, they are parametrized by an ind-scheme which is a limit of schemes of finite type over $k$.

It is inconvenient to think of lattice classes as equivalence classes. If $L \subset K^{n}$ is a maximal lattice, then $\operatorname{Hom}_{\mathcal{O}}(L, L)=\operatorname{End}(L)$ is naturally an $\mathcal{O}$-subalgebra of $M_{n}(K)=$ $\operatorname{Hom}_{K}\left(K^{n}, K^{n}\right)$. If two lattices are equivalent it is clear that their endomorphism algebras are equal. Conversely if $L$ and $L^{\prime}$ are two lattices with equal endomorphism algebras, and $E=\operatorname{End}(L)=\operatorname{End}\left(L^{\prime}\right)$, the two lattices are both representations of $E$. Hence there is an $E$-isomorphism, $\theta$, from $L$ to $L^{\prime}$. Localize and $\theta$ becomes an $M_{n}(K)$-automorphism of $K^{n}$ and hence multiplication by a scalar. That is, the additive lattice classes correspond injectively to the finite central simple $\mathcal{O}$-subalgebras of $M_{n}(K)$. Finally if $E$ is any central simple $\mathcal{O}$ subalgebra of $M_{n}(K)$ and $L$ is any maximal lattice, $E L=N$, being a quotient of a tensor product of finites, is $\mathcal{O}$-finite and hence a lattice and an $E$-module. It follows that $E=$ $\operatorname{End}(N)$. That is, additive lattice classes are in bijective correspondence with the central simple $\mathcal{O}$-subalgebras of $M_{n}(K)$.

Definition 21. A lattice class of degree $n$ in $M_{n}(K)$ is a finite central simple $\mathcal{O}$ subalgebra of $M_{n}(K)$ of rank $n^{2}$. If $M$ is a lattice class of degree $n$ in $M_{n}(K)$ then the set of maximal lattices in $K^{n}$ which are $M$-modules is called the associated additive lattice class of $M$. An element of this class is called an additive representative of $M$. Finally, a finite unital $\mathcal{O}$-subalgebra of $M_{n}(K)$ which is a special lattice with respect to the standard lattice, $M_{n}(\mathcal{O})$, will be called a special subalgebra of $M_{n}(K)$.

Recall that by Proposition 3, for $r$ and $s$ positive, there is an algebraic, bilinear morphism of group schemes, $\mu_{r, s}: p^{-r} W_{k}^{(s)} \times_{k} p^{-s} W_{k}^{(r)} \rightarrow p^{-r-s} W_{k}$ which is just multiplication on points. Here the superscripts in parentheses indicate Frobenius covers. Writing $\mathcal{O}$ for $W_{k}$, this can be applied to $p^{-r} M_{n}(\mathcal{O})$ and even to the quotient, $p^{-r} M_{n}(\mathcal{O}) / p^{\left(n^{2}-1\right) r} M_{n}(\mathcal{O})$. Since addition is defined without passing to a Frobenius cover, Proposition 3 and the properties of addition imply that we may define an algebraic map $m_{r}: p^{-r} M_{n}^{(r)} \times_{k} p^{-r} M_{n}^{(r)} \rightarrow p^{-2 r} M_{n}$. Let $X=\mathbb{L a t}_{r}^{n^{2}}(K)$. Identify $K^{n^{2}}$ with $M_{n}(K)$ and let $M_{n}=M_{n}(\mathcal{O})$ be the standard lattice in this $K$-vector space. Now $M_{n}(K)$ admits a representation on $K^{n}$. Let $F \subset K^{n}$ be a standard lattice in $K^{n}$ such that $\operatorname{End}_{\mathcal{O}}(F)=M_{n}$. Taking products and using the natural properties of 
Frobenius covers we obtain a commutative diagram which we will use in the next proof:

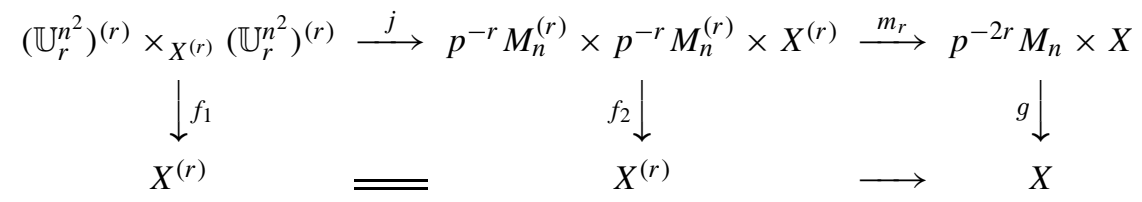

Proposition 9. Let $X=\mathbb{L a t}_{r}^{n^{2}}(K)$ be the scheme of lattices of rank $n^{2}$ and height at most $r$ in $M_{n}(K)$. Let $M_{n}(\mathcal{O})$ be the standard lattice. Let $\mathbb{U}_{r}^{n^{2}}$ be the universal family over $X$.

(1) There is a closed subscheme of $X$, which we denote $\mathbb{C} L_{n}^{r}(K)$, which consists of exactly those points, $x$, in $X$ such that the fiber of $\mathbb{U}_{r}^{n^{2}}$ over $x$ is a special lattice and a subalgebra with unit in $M_{n}(K)$.

(2) Let $\mathbb{M}_{n}^{r}=\mathbb{U}_{r}^{n^{2}} \mid \mathbb{C} L_{n}^{r}(K)$. Then $\mathbb{M}_{n}^{r}$ is a flat family of special $\mathcal{O}$-subalgebras of $M_{n}(K)$ and if $Y$ is any $k$-scheme furnished with a flat scheme, $T$, of special subalgebras of $M_{n}(K)$, then there is a unique map, $f: Y \rightarrow \mathbb{C} L_{n}^{r}(K)$, so that $T=Y \times{ }_{\mathbb{C} L_{n}^{r}(K)} \mathbb{M}_{n}^{r}$.

PROOF. Write $M_{X}=M_{n} \times_{k} X$. Then, $M_{X} \backslash \mathbb{U}_{r}^{n^{2}}$ is open. Hence $\left(m_{r} \circ j\right)^{-1}\left(M_{X} \backslash \mathbb{U}_{r}^{n^{2}}\right)$ is an open set. Denote this open set $P$. Now $\mathbb{U}_{r}^{n^{2}}$ is flat over $X$ and so $\left(\mathbb{U}_{r}^{n^{2}}\right)^{(r)}$ is flat over $X^{(r)}$. This implies that $f_{1}$ is flat as well. We would like to conclude that $f_{1}\left(\left(m_{r} \circ j\right)^{-1}\left(M_{X} \backslash \mathbb{U}_{r}^{n^{2}}\right)\right)$ is open. We may not because $M_{X}, \mathbb{U}_{r}^{n^{2}}$ and the other schemes involved are not of finite type over $X$. Consider $p^{-r} M_{n}$. It can be thought of as the spectrum of the ring of poynomials in the countable set of variables, $\left\{x_{i, j, s}:-r \leq i, j \leq n, s \in \mathbb{Z}, s \geq-r\right\}$. The point with coordinates $x_{i, j, s}$ corresponds to the matrix with entries, $u_{i, j}$ where $u_{i, j}=\sum_{-r}^{\infty} \xi\left(x_{i, j, s}\right)^{p^{-s}} p^{s}$. From this description, it is clear that $p^{-r} M_{n}=\left(p^{-r} M_{n} / p^{\left(n^{2}-1\right) r} M_{n}\right) \times_{k} p^{\left(n^{2}-1\right) r} M_{n}$ and that this isomorphism may be taken to be a $p^{\left(n^{2}-1\right) r} M_{n}$-equivariant isomorphism of varieties for the additive structure. This observation can be extended to the product, $p^{-r} M_{n} \times{ }_{k} p^{-r} M_{n}$ as well. Now it is clear that $\left(m_{r} \circ j\right)^{-1}\left(p^{-r} M_{X} \times p^{-r} M_{X}\right)$ is $p^{n^{2}-1} M_{n}$-invariant. This is so because $(u, v) \in P$ if and only if $u, v \in L$ for some special lattice, but $u v \notin L$. If $\left(u+p^{n^{2}-1} z\right)\left(v+p^{n^{2}-1} w\right) \in L$, it would follow that $u v \in L$ because every lattice in $\mathbb{L}_{r} t_{r}^{n^{2}}(K)$ contains $p^{n^{2}-1} M_{n}$.

There is a natural projection:

$$
p^{-r} M_{n} \times p^{-r} M_{n} \times X \rightarrow\left(p^{-r} M_{n} / p^{n^{2}-1} M\right) \times\left(p^{-r} M_{m} / p^{n^{2}-1} M_{n}\right) \times X .
$$

Consequently, the invariance of $P$ noted above implies that $P$ is the inverse image of an open set, $\bar{P} \subseteq\left(p^{-r} M_{n} / p^{n^{2}-1} M_{n}\right) \times\left(p^{-r} M_{n} / p^{n^{2}-1} M_{n}\right) \times X$. It follows that $f_{1}(P)$ is the image of $\bar{P}$ in $X$ under the natural projection from $\left(\mathbb{U}_{r}^{n^{2}} / p^{n^{2}-1} M_{n}\right)^{(r)} \times_{X}\left(\mathbb{U}_{r}^{n^{2}} / p^{n^{2}-1} M_{n}\right)^{(r)}$. This last scheme is flat and of finite type over $X^{(r)}$ and so its image is open. Since the Frobenius is a homeomorphism, its image in $X$ is open

This image is exactly the set of lattices, $N \subset p^{-r} M_{n}(K)$, which contain two elements $u$ and $v$ so that $u v \notin N$. That is, it is the set of special lattices which are not closed under multiplication. Hence its complement, which is closed, is exactly the set of points whose fibers 
are special lattices closed under multiplication. The Frobenius cover is a homeomorphism and so its image in $X$ is a closed subset consisting exactly of those lattices closed under multiplication. Denote this set $Z$. (Over $Z$, the multiplication map is not defined.) Let $C=\mathcal{O} \cdot 1$ denote the center of $M_{X}$. Then $C \subset \mathbb{U}_{r}^{n^{2}}$. Both $C$ and $g^{-1}(Z)$ are closed $k^{*}$-stable subsets of $\mathbb{U}_{r}^{n^{2}}$ and so by Corollary 1, the image of $C \cap g^{-1}(Z)$ in $X$ is closed. This is exactly the set of special lattices in $p^{-r} M_{n}$ which are algebras with unit.

The second statement follows from the universal mapping property for $\mathbb{L a t}_{r}^{n^{2}}(K)$. There is a unique map satisfying the asserted condition and the fact that the fibers of $T$ are subalgebras of $M_{n}(K)$ implies that the image of $T$ is in $\mathbb{C} L_{n}^{r}$.

LEMMA 9. Let $L \subseteq M_{n}(K)$ be a special lattice which is also a subalgebra with unit. Then there is a matrix $\beta \in G L(n, K)$ so that $L=\beta M_{n} \beta^{-1}$. Consequently, $\mathbb{M}_{n}^{r}$ is a scheme of fiberwise trivial central simple $\mathcal{O}$-algebras and so a family of lattice classes parametrized by $\mathbb{C} L$.

Proof. Consider the product, $L F \subset K^{n}$. It is a surjective image of $L \otimes_{\mathcal{O}} F$ and so is finite over $\mathcal{O}$ and hence free of rank $n$. Hence for some non-singular matrix, $\beta \in$ $G L(n, K), L F=\beta F$. Further $L F$ is certainly a left $L$-module. Hence $L \subseteq \beta M_{n} \beta^{-1}$. Now notice that the determinant of left multiplication by $\beta$ is $\operatorname{det}(\beta)^{n}$ and the same is true of right multiplication. Hence, conjugation is special linear and so $\bigwedge^{n^{2}}\left(\beta M_{n} \beta^{-1}\right)=\bigwedge^{n^{2}} M_{n}$. Since $L$ is special, $\bigwedge^{n^{2}} L=\bigwedge^{n^{2}} M_{n}$. On the other hand $\bigwedge^{n^{2}} L=p^{c} \bigwedge^{n^{2}}\left(\beta M_{n} \beta^{-1}\right)$ where $c$ is the length of $\left(\beta M_{n} \beta^{-1}\right) / L$. It follows that $L=\beta M_{n} \beta^{-1}$. The last statement of the lemma follows trivially.

REMARK 3. A fiberwise trivial family may not be itself trivial. The constructions of the lemma are not applicable at any but the closed points of $\mathbb{C} L$. This observation also applies to the following.

LEMMA 10. Let $M$ be a lattice class of degree $n$ in $M_{n}(K)$ of height at most $m$. Then $M \supseteq p^{m} M_{n}$. Moreover $M$ admits an additive representative, $L$, such that $p^{-m} F \supseteq L \supseteq$ $p^{m} F$.

Proof. First note that $M$ is a conjugate of $M_{n}$ by some $\beta \in G L(n, K)$. By the Iwahori decomposition, we may write $\beta=b \gamma c$ where $\gamma$ is diagonal with powers of $p$ on the diagonal and $b$ and $c$ are in $G L(n, \mathcal{O})$ but with subdiagonal entries divisible by $p$. Since $c M_{n} c^{-1}=$ $M_{n}, M=b \gamma M_{n} \gamma^{-1} b^{-1}$. For any $\mathcal{O}$-submodule of $M_{n}(K), U$, clearly $p^{r} M_{n} \supseteq U p^{s} M_{n}$ if and only if $p^{r} M_{n} \supseteq b^{-1} U b \supseteq p^{s} M_{n}$. Hence it suffices to show that $\gamma M_{n} \gamma^{-1} \supseteq p^{m} M_{n}$.

For the same reason we can see that $p^{-m} M_{n} \supseteq M$ if and only if $p^{-m} M_{n} \supseteq \gamma M_{n} \gamma^{-1}$ and so the hypothesis allows us to assume this so. Write $\gamma=\operatorname{diag}\left(p^{r_{1}}, \ldots, p^{r_{n}}\right)$. Then if $\alpha=\left(a_{i, j}\right)$ is a matrix, $\gamma \alpha \gamma^{-1}$ has $(i, j)$-entry $p^{r_{i}-r_{j}} a_{i, j}$. Let $E_{i, j}$ be the matrix whose only non-null entry is 1 in the $(i, j)$-place. These are a basis of $M_{n}$. Hence the matrices $\gamma E_{i, j} \gamma^{-1}$ are a basis for $\gamma M_{n} \gamma^{-1}$. But $\gamma E_{i, j} \gamma^{-1}=p^{r_{i}-r_{j}} E_{i, j}$. If for all $i, j, r_{i}-r_{j} \geq-m$, then it is equally true that $r_{i}-r_{j} \leq m$. This proves that $p^{-m} M_{n} \supseteq M \supseteq p^{m} M_{n}$, which is the 
first assertion of the lemma. For the second let $L=M F$ and establish the final assertion by multiplying the inclusions of the first assertion by $F$.

LEMMA 11. Let $Y$ be a $k$-scheme and let $h: \mathbb{L} \rightarrow Y$ be a flat scheme of rank $n$ sublattices of $p^{-r} F$ of index $q$. Then the scheme of endomorphisms of $\mathbb{L}$ is a flat family of unital subalgebras of $M_{n}(K)$ lying in $p^{-n r-q} M_{n}$.

PROOF. Let $M$ be the endomorphism ring of some fiber of $\mathbb{L}$, which we designate $L$. Since $p^{-r} F \supseteq L \supseteq p^{q+(n-1) r} F$ we may divide these inclusions by the corresponding $p$ powers to obtain $p^{-q-(n-1)) r} L \supseteq F \supseteq p^{r} L$. Hence if $\theta(L) \subseteq L, p^{q+(n-1)} \theta(F) \subseteq \theta(L) \subseteq$ $L \subseteq p^{-r} F$. That is, $p^{q+(n-1) r} \theta(F) \subseteq p^{-r} F$ hence $\theta(F) \subseteq p^{-q-n r} F$. Hence $M \subseteq$ $p^{-q-n r} M_{n}$.

Let $N=p^{-n r-q} M_{n} \times \mathbb{L}$. Matrix multiplication gives a map, $\alpha: N^{(-n r-q)} \rightarrow$ $p^{-2 n r-2 q} M_{n}$. (Note the Frobenius cover on the right.) Let $U=\alpha^{-1}\left(p^{-2 n r-2 q} M_{n} \backslash \mathbb{L}\right)$. Then $U$ is open in $\left(p^{-n r-q} M_{n} \times \mathbb{L}\right)^{(-n r-q)}$. Since $h$ is flat, the projection of $\left(p^{-n r-q} M_{n} \times\right.$ $\mathbb{L})^{(-n r-q)}$ on $\left(p^{-n r-q} M_{n} Y\right)^{(-n r-q)}$ is flat and invariant under a suitable $p$ multiple of the lattices involved. Hence the argument used in the proof of Proposition 9 applies and so $(\mathrm{id} \times h)(U)$ is open in $p^{-2 n r-2 q} M_{n} \times Y$ and so its complement is closed. The complement of the image of $N$ is exactly the family of endomorphism algebras of $\mathbb{L}$. It can be seen to be flat by considering its quotient by a suitable $p$ multiple of $M_{n}$ as in the proof of Proposition 9 .

Consider $\mathbb{G L a t}_{r, q}^{n}$. Its universal bundle of lattices, $\mathbb{G U}_{r, q}^{n}$ is a bundle of lattices of index $q$. By Lemma 9, its endomorphism bundle is a flat family of central simple $\mathcal{O}$-subalgebras of $M_{n}(K)$ lying in $p^{-n r-q} M_{n}$. Hence there is a natural map from $\mathbb{G} \mathbb{L} a t_{n}^{r, q}$ to $\mathbb{C} L_{n}^{r n+q}$. Composing this with the natural inclusion, there is a natural map to $\mathbb{C} L_{n}^{m}$ for each $m \geq n r+q$. Write $\Phi_{n, m}^{r, q}$ for this map. We will find use for it in the discussion in this section.

Consider $\mathbb{G L a t}_{r, q}^{n}$ and suppose that $L$ corresponds to a point in it. That is, $L$ is of type $\left(r_{1}, \ldots, r_{n}\right)$ and the $r_{i}$ satisfy the conditions, $-r \leq r_{i} \leq n r, r_{1}+\cdots+r_{n}=q$ and $-n r \leq$ $q \leq r$. We will refer to $\operatorname{End}_{\mathcal{O}}(L)$ as the class of $L$. Notice that the index of $p L$ is $q+n$. Then $p L \notin \mathbb{G L a t}_{r}^{n}$ if and only if $r_{i}+1>n r$ for at least one $i$. But then the $r_{j}$ satisfy, $r_{1}+\cdots+r_{n} \leq r$ and $r_{i}=n r$. This is only possible if $r_{j}=-r, j \neq i$ and $L$ is special.

DEFINITION 22. Let $v$ be an element of the integers modulo $n$ and let $v_{0}$ be a nonnegative integer less than $n$ representing it. Write $\mathbb{P L a t}_{r, v}^{n}$ for the image of $\mathbb{G L a t}{ }_{r, \nu_{0}}^{n}$ in $\mathbb{C} L_{n}^{n(r+1)}$ under $\Phi_{n, n(r+1)}^{r, v_{0}}$. We shall refer to this scheme as the scheme of $r$-representible projective lattice classes of index class $v$. Finally write $\mathbb{P L a t}{ }^{n}(v)$ for the direct limit $\lim _{r} \mathbb{P L a t}{ }_{r, v}^{n}$.

While it is clear that $\Phi_{n, n(r+1)}^{r, \nu_{0}}$ is injective on closed points, it is not clear that it is separable. Hence one cannot use it to construct a universal family of additive lattice classes. However the lattice family $\mathbb{M}_{r}^{n} \mid \mathbb{P} \mathbb{L a t}_{r, v}^{n}$ is a universal family and so the direct limit of these families over $r$ is a universal family of lattice classes on $\mathbb{P L a t}^{n}(v)$. Write $\mathbb{M}_{n}(v)$ for this family.

3.4. The geometry of the lattice spaces: The spaces $\mathbb{L}_{a} a_{r}^{n}(K)$ and the varieties derived from them do not behave well with respect to the group action. Since $\mathbb{L}_{\mathrm{at}}{ }_{r}^{n}(K)$ contains points 
with coordinates constructed from Witt vectors of value $-r$, it is only the $r$ 'th Frobenius cover of $S L(n, \mathcal{O})$ which acts on it algebraically. To remedy this we reparametrize the space so that $S L(n, \mathcal{O})$ operates on it independently of $r$. In this section, $\operatorname{SL}(n, \mathcal{O}), G L(n, \mathcal{O})$ and $P G L(n, \mathcal{O})$ denote these groups with their imperfect structures. That is, they are just proalgebraic groups over $k$. Moreover $B$ is the Iwahori subgroup consisting of elements in $S L(n, \mathcal{O})$ with entries of strictly positive value below the diagonal.

Consider the verschiebung. Recall that if $k\left[x_{0}, \ldots, x_{j}, \ldots\right]$ is the coordinate ring of the scheme of Witt vectors, the verschiebung is the endomorphism, $\mathfrak{v}$, defined by the algebra map, $v_{0}\left(x_{i}\right)=x_{i-1}, v_{0}\left(x_{0}\right)=0$. It induces a separable additive endomorphism of the scheme of Witt vectors. If $\phi$ is the $k$-morphism defined by $\phi\left(x_{i}\right)=x_{i}^{p}$ then, though $\phi$ is an inseparable endomorphism of the polynomial domain, on Witt vectors, that is to say $k$-points, it induces the Frobenius automorphism of $\mathcal{O}$ and of the Hilbert class field. In addition, $v_{0} \circ \phi$ is the contravariant morphism of coordinate rings corresponding to multiplication by $p$.

The Witt vectors are embedded in the topological scheme, $K^{+}$, as the common zeros of the ideal generated by all the $x_{i}$ with $i<0$. Consequently, $v^{0}$ extends to the complete coordinate ring of $K^{+}$by setting $v_{0}\left(x_{i}\right)=x_{i-1}$ for all $i$. Let $\phi$ be the map of coordinate rings, $\phi\left(x_{i}\right)=x_{i}^{p}$ for both $K^{+}$and the Witt vectors. As above this gives an inseparable additive endomorphism of the scheme of Witt vectors.

Write $\mathfrak{v}$ for the verschiebung viewed as a separable endomorphism of the scheme of Witt vectors or of its ring of points. On points, $\mathbf{a}=\left(a_{0}, a_{1}, \ldots, a_{i}, \ldots\right), \mathfrak{v}(\mathbf{a})=\left(0, a_{0}, a_{1}, \ldots\right)$. The verschiebung extends uniquely to either $K^{+}$or some overmodule of $\mathcal{O}$ in $K^{+}$. We write it $\mathfrak{v}$ as well. The co-morphism corrsponding to it is just given by $v_{0}\left(x_{i}\right)=x_{i-1}$ for all $i$. Since this map preserves the ideal of functions vanishing on $p^{r} \mathcal{O}$ for all $r$, this map on the fractions restricts to the map defined above on $\mathcal{O}$. Hence there is a map, $\mathfrak{v}^{r}: p^{-r} \mathcal{O} \rightarrow \mathcal{O}$ defined by $v_{0}^{r}\left(x_{i}\right)=x_{i-r}, i \geq 0$ and a map $\mathfrak{v}^{s}: K^{+} \rightarrow K^{+}$defined by $v_{0}^{s}\left(x_{i}\right)=x_{i-s}$ and these may be assumed to be coherent under restriction.

Multiplication by $p$ is an $\mathcal{O}$-homomorphism of modules. Thus $\mathfrak{v}(\phi(\mathbf{a}) \phi(\mathbf{b}))=\mathbf{a} \mathfrak{v}(\phi(\mathbf{b}))$. We may pass to a Frobenius cover so that each $\mathbf{b}$ may be written as $\phi\left(b^{\prime}\right)$. Doing so, we obtain:

$$
\mathbf{a v}(\mathbf{b})=\mathfrak{v}(\phi(\mathbf{a}) \mathbf{b}) .
$$

Consider the standard module, $F \subseteq K^{n}$, and fix once and for all an ordered basis for $F,\left\{e_{1}, \ldots, e_{n}\right\}$. We may use this basis, which we view as an $\mathcal{O}$-structure, to define a verschiebung on $K^{n}$. Just set $\mathfrak{v}_{F}\left(\sum a_{i} e_{i}\right)=\sum \mathfrak{v}\left(a_{i}\right) e_{i}$. Then $\mathfrak{v}_{F}$ is $\phi$-semilinear. That is, it satisfies:

$$
\begin{aligned}
& \mathfrak{v}_{F}(u+w)=\mathfrak{v}_{F}(u)+\mathfrak{v}_{F}(w), \\
& \mathfrak{v}_{F}(\phi(\mathbf{a}) u)=\mathbf{a v}_{F}(u) .
\end{aligned}
$$

If $\alpha$ is an $(n \times n)$-matrix over $\mathcal{O}$, this implies that:

$$
\mathfrak{v}_{F}\left(\phi_{F}(\alpha) u\right)=\alpha \mathfrak{v}_{F}(u) .
$$

Here the $\phi_{F}$ on $\alpha$ is given by $\phi_{F}\left(a_{i, j}\right)=\left(\phi\left(a_{i, j}\right)\right)$ and it is evidently dependent on the choice of basis. Notice that, for $G_{0}=S L(n, \mathcal{O})$ and for $B$ equal to either of the two 
obvious Iwahori subgroups in $G_{0}, \phi_{F}\left(G_{0}\right)=G_{0}$ and $\phi_{F}(B)=B$. Thus $\phi$, though it is a purely inseparable endomorphism of pro-algebraic groups, is just an automorphism of groups of $k$-points.

The pro-algebraic group, $S L(n, \mathcal{O})$ operates algebraically on $F$. In particular, the second equation of (3.2) implies that the orbit of an element of $F$ under $S L(n, \mathcal{O})$ is the image under the verscheibung of an orbit.

Recall that, in the terminology of Proposition 6. $\mathbb{L}(q, m ; N)$ denotes the scheme of lattices in $p^{q} F / p^{m} F$ of $k$-dimension, $N$. Write $\mathbb{L}_{r}(F)$ for $\mathbb{L}(0, n r ; n(n-1) r)$ and write $\mathbb{U}_{r}(F)$ for its universal family of group subschemes of $F / p^{n r} F$. It is quite easy to see that since these schemes are constructed by applying standard methods of algebraic geometry over $k$ to $\left(K^{+}\right)^{n}$, that morphisms involving $\phi$ or multiplication by powers of $p$ which are defined on them are $k$-morphisms.

LEMmA 12. There is a canonical isomorphism, $\tilde{\mathfrak{v}}_{F}^{r}: \mathbb{L}_{\mathrm{L}} \mathrm{t}_{r}^{n}(K) \rightarrow \mathbb{L}_{r}(F)$. Moreover $\tilde{\mathfrak{v}}_{F}^{r}$ carries $S L(n, \mathcal{O})$-orbits and $B$-orbits under the action in the complete perfect category to $S L(n, \mathcal{O})$-orbits and $B$-orbits in the algebraic category. Moreover, for each $r$, the following diagram commutes:

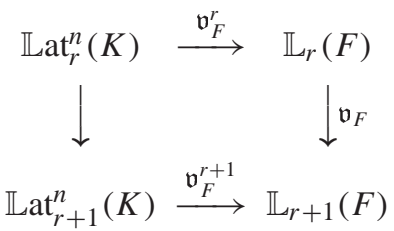

ProOF. The morphism is the one which carries the lattice $L \subseteq p^{-r} F$ to $\mathfrak{v}_{F}^{r}(L)$. The semi-linearity insures that this is a lattice and it is evidently of the correct codimension. Since it is functorial, it is a morphism of lattice varieties. By abuse of language we use the same symbol for the morphism of lattice varieties. The semi-linearity also insures, as we have remarked, that $\mathfrak{v}_{F}^{r}$ carries orbits to orbits and that all orbits are images of orbits.

It is convenient to introduce a limit of the schemes, $\mathbb{L}_{r}(F)$.

DEFinition 23. Write $\mathfrak{v}_{F}^{s}$ for the $k$-morphism of schemes from $\mathbb{L}_{r}(F)$ to $\mathbb{L}_{r+s}(F)$ which sends $L$ to $\mathfrak{v}_{F}^{s}(L)$. Let $\mathbb{L}^{0}(F)=\lim _{\longrightarrow} \mathbb{L}_{r}(F)$ where the morphisms are the maps $\mathfrak{v}_{F}^{s}: \mathbb{L}_{r}(F) \rightarrow \mathbb{L}_{r+s}(F)$. We shall call this the space of lattices of index 0 in $F$. We also write $\mathfrak{v}_{F}^{s}$ for the obvious endomorphism of $\mathbb{L}^{0}(F)$ induced by the constant directed family of morphisms each of which is equal to $\mathfrak{v}_{F}^{S}$.

The effect of Lemma 12 is that we may analyze orbit structure in $\mathbb{L a t}_{r}^{n}(K)$ under the standard action. This action may actually be twisted by the Frobenius but it is immaterial. Inclusions in closures of other orbits, computations of dimension and other routine computations can be done as though all our actions are algebraic and as though the Frobenius twist is unnecessary. This is the case because $\mathfrak{v}_{F}^{r}$ transforms these orbits into algebraic orbits in $\mathbb{L}_{r}(F)$. The disadvantage of passing to $\mathbb{L}^{0}(F)$ in discussing orbit structure is that the representative of an orbit is different in each $\mathbb{L}_{r}(F)$. The orbit corresponding to the diagonal 
element $\exp _{p}\left(r_{1}, \ldots, r_{n}\right)$ in $\mathbb{L}^{n}(K)$ is the orbit of $\exp _{p}\left(r+r_{1}, \ldots, r+r_{n}\right)$ in $\mathbb{L}_{r}(F)$. (Recall that $\exp _{p}\left(r_{1}, \ldots, r_{n}\right)$ is $\operatorname{diag}\left(p^{r_{1}}, \ldots, p^{r_{n}}\right)$.) For this reason we describe orbits in $\mathbb{L}_{r} t_{r}^{n}(K)$.

At this point it is germaine to review the somewhat complicated situation at hand. The group $S L(n, K)$ admits a structure as a group object in the category of perfect topological schemes. In consequence its group of points, which we also write $S L(n, K)$, operates as a set of automorphisms on $\mathbb{L a t}^{n}(K)$ and $\mathbb{L}^{0}(F)$. These actions, however, just cannot be realized as morphic actions in the category of ind-schemes. On the other hand there is an algebraic action of the pro-algebraic group, $S L(n, \mathcal{O})$, on the ind-schemes $\mathbb{L}_{r}(F)$ and their limit, $\mathbb{L}^{0}(F)$. Further, for each $r$, this action is essentially isomorphic, via the verscheibung, to the action of a suitable Frobenius cover of $S L(n, \mathcal{O})$ on $\operatorname{Lat}_{r}^{n}(K)$.

Let $B$ be the Iwahori subgroup of $S L(n, \mathcal{O})$ with subdiagonal entries of positive value. Write $\bar{B}$ for the image of this group in $\operatorname{PGL}(n, \mathcal{O})$. Let $N$ denote the Cartan subgroup of $S L(n, K)$ and let $\bar{N}$ denote the Cartan subgroup of $P G L(n, K)$. Let $T$ and $\bar{T}$ denote the maximal tori contained in $N$ and $\bar{N}$, respectively. Then, $S L(n, \mathcal{O})$ and $P G L(n, \mathcal{O})$ are good (bon) maximal bounded subgroups. Write $\Gamma$ and $\bar{\Gamma}$ for the groups of one-parameter subgroups of $T$ and $\bar{T}$, respectively. Then $\Gamma$ can be thought of as the co-root lattice, while $\bar{\Gamma}$ can be thought of as the lattice of dual weights. Then if $W^{*}$ is the Weyl group of the Tits system in $S L(n, K)$ and $\bar{W}^{*}$ that associated to $P G L(n, K)$, we may write $W^{*}=\Gamma \cdot W$ and $\bar{W}^{*}=\bar{\Gamma} \cdot W$ where $W$ is the Weyl group associated to the special point 0 . It is the same for both groups and is generated by the reflections, $s_{1}, \ldots, s_{l}$. Let $s_{0}$ denote the additional reflection in $W^{*}$ and let $\bar{s}_{0}$ be the one in $\bar{W}^{*}$. Write $\Gamma^{+}$and $\bar{\Gamma}^{+}$for the intersections of $\bar{\Gamma}$ and $\Gamma$ with the dominant chamber.

There are at least three double coset decompositions of interest. Two are well known; the third is easily derived from well known facts and has been frequently observed. The first of these is the standard Bruhat decomposition associated to the Tits systems, $\left(B, N, s_{0}, s_{1}, \ldots, s_{l}\right)$ and $\left(\bar{B}, \bar{N}, \bar{s}_{0}, s_{1}, \ldots, s_{l}\right)$ :

$$
\begin{gathered}
S L(n, K)=\bigcup_{\gamma \in \Gamma, w \in W} B \gamma \cdot w B, \\
P G L(n, K)=\bigcup_{\gamma \in \bar{\Gamma}, w \in W} \bar{B} \gamma \cdot w \bar{B} .
\end{gathered}
$$

The second pair of decompositions corresponds to the classical Birkhoff decomposition. To state it conveniently write $G_{0}=S L(n, \mathcal{O})$ and $\bar{G}_{0}=P G L(n, \mathcal{O})$.

$$
\begin{gathered}
S L(n, K)=\bigcup_{\gamma \in \Gamma^{+}} G_{0} \gamma G_{0}, \\
P G L(n, K)=\bigcup_{\gamma \in \bar{\Gamma}^{+}} \bar{G}_{0} \bar{\gamma} \bar{G}_{0} .
\end{gathered}
$$

The last decomposition of interest depends on an observation. Consider the union $\bigcup_{w \in W} B \gamma w B=C(\gamma)$ for a fixed $\gamma$. If $s_{i}, i>0$ is a reflection in $W$, then $w B s_{i} \subset$ 
$B w B \cup B w s_{i} B$. This is just property T3 in the definition of a Tits system in [Bo]. It follows that $C(\gamma)$ is stable under right multiplication by $B$ and right multiplication by the $s_{i}$. That is, $C(\gamma)$ is stable under right multiplication by $W$ and $B$ and so by $G_{0}$ which is generated by them. Thus $C(\gamma) \supset B \gamma G_{0}$. On the other hand $w B \subset G_{0}$ for each $w$ and so $C(\gamma) \subset B \gamma G_{0}$ for each $\gamma$ and so $C(\gamma)=B \gamma G_{0}$. Finally it is clear that if $\gamma \neq \lambda$ then the sets $\{\gamma w: w \in W\}$ and $\{\lambda w: w \in W\}$ are disjoint as are their $B$-double cosets. The same argument shows that the corresponding union is $\bar{B} \gamma \bar{G}_{0}$ in $\operatorname{PGL}(n, K)$. Hence:

$$
\begin{gathered}
S L(n, K)=\bigcup_{\gamma \in \Gamma} B \gamma S L(n, \mathcal{O}), \\
P G L(n, K)=\bigcup_{\gamma \in \bar{\Gamma}} \bar{B} \gamma P G L(n, \mathcal{O}) .
\end{gathered}
$$

The decompositions, (3.4)-(3.7), actually give the orbit structure for $\mathbb{L a t}^{n}(K)$ and $\mathbb{P L a t}(n, K)$ under the Iwahori subgroup, $B$ (respectively $\bar{B}$ ) and a particular good maximal parahoric subgroup, $G_{0}$ (respectively $\bar{G}_{0}$ ). Let $x_{0} \in \mathbb{L}^{n}{ }^{n}(K)$ be the point corresponding to the standard lattice and let $\bar{x}_{0}$ denote the corresponding point in PLat $(n, K)$. We restrict our attention to $S L(n, K)$. Write $\mathbf{G}$ for this group. Then the double coset representations, 3.4 and 3.6, reduce mod $G_{0}$ to give the orbit decompositions of $\mathbb{L a t}^{n}(K)$ under $G_{0}$ and $B$, respectively. Take for $B$ the set of elements in $G_{0}$ which are congruent to an upper triangular matrix modulo $p$. We may take for $\Gamma$ the set of matrices, $\left\{\Delta\left(p^{r_{1}}, \ldots, p^{r_{n}}\right): \sum r_{i}=0\right\}$ where $\Delta\left(x_{1}, \ldots, x_{n}\right)$ signifies the diagonal matrix with diagonal entries $x_{1}, \ldots, x_{n}$. We have introduced the notation $\exp _{p}\left(r_{1}, \ldots, r_{n}\right)$ for $\Delta\left(p^{r_{1}}, \ldots, p^{r_{n}}\right)$. The positive simple roots are the characters, $\alpha_{i, i+1}$, defined by $\alpha_{i, i+1}\left(\Delta\left(t_{1}, \ldots, t_{n}\right)\right)=t_{i} t_{i+1}^{-1}$. The canonical pairing between characters, $\chi$, and $T$-points is just given by $\langle\chi, t\rangle=v(\chi(t))$ where $v$ is the $p$-adic value. Thus $\left\langle\alpha_{i, j}, \exp _{p}\left(r_{1}, \ldots, r_{n}\right)\right\rangle=r_{i}-r_{j}$. Hence $\Gamma^{+}$is just the set of elements in $\Gamma$ for which $r_{1} \geq r_{2} \geq \cdots \geq r_{n}$

Since the stabilizer of $x_{0}$ in $\mathbb{L a t}^{n}(K)$ is $G_{0},(3.6)$ and (3.8) imply that the $G_{0}$ - and $B$-orbit decompositions of $\mathbb{L a t}^{n}(K)$ are:

$$
\begin{aligned}
& \mathbb{L a t}^{n}(K)=\bigcup_{\gamma \in \Gamma^{+}} G_{0} \gamma x_{0}, \\
& \mathbb{L a t}^{n}(K)=\bigcup_{\gamma \in \Gamma} B \gamma x_{0} .
\end{aligned}
$$

The $\mathbf{G}$-stabilizer of $x_{0}$ is $G_{0}$ and so the $\mathbf{G}$-stabilizer of $\gamma x_{0}$ is $\gamma G_{0} \gamma^{-1}$. Write $G_{0}(\gamma)$ and $B(\gamma)$ for the $G_{0^{-}}$and $B$-stabilizers of $\gamma x_{0}$, respectively. Then:

$$
\begin{aligned}
G_{0}(\gamma) & =G_{0} \cap \gamma\left(G_{0}\right) \gamma^{-1}, \\
B(\gamma) & =B \cap \gamma G_{0} \gamma^{-1} .
\end{aligned}
$$

Suppose that $\gamma=\exp _{p}\left(r_{1}, \ldots, r_{n}\right)$. Then $\gamma G_{0} \gamma^{-1}$ is the set of matrices $\left(x_{i, j}\right)$ such that $v\left(x_{i, j}\right) \geq r_{i}-r_{j}$. Since $G_{0}$ is the set of matrices in $\mathbf{G}$ all of whose entries have non-negative 
value and $B$ is the set of matrices in $G_{0}$ with subdiagonal elements of value at least one, these groups can be described precisely:

$$
\begin{aligned}
G_{0}(\gamma) & =\left\{\left(x_{i, j}\right) \in G_{0}:\right. \\
B(\gamma) & \left.v\left(x_{i, j}\right) \geq \sup \left\{0, r_{i}-r_{j}\right\}\right\}, \\
& v\left(x_{i, j}\right) \in B\left(x_{i, j}\right) \geq \sup \left\{0, r_{i}-r_{j}\right\}, \quad i<j ; \\
v\left(x_{i, j}\right) & \left.\geq \sup \left\{1, r_{i}-r_{j}\right\}, i>j\right\} .
\end{aligned}
$$

This description is of particular importance in view of the following for which we believe no proof is necessary:

LEMMA 13. For each pair $i, j$ with $i$ and $j$ between 1 and $n$, suppose that $m_{i, j}$ is a non-negative integer. Let $H$ be the set of matrices, $\left(x_{i, j}\right)$, in $\operatorname{SL}(n, \mathcal{O})$ such that $v\left(x_{i, j}\right) \geq$ $m_{i, j}$. Suppose that $H$ is a subgroup of the group of invertible matrices. Then the following hold.

(1) The coset space, $G_{0} / H$ is a finite dimensional homogeneous space of dimension, $\sum_{i, j} m_{i, j}$

(2) If $m_{i, j} \geq 1$ whenever $i>j$, then $H \subseteq B$ and $B / H$ is a finite dimensional homogeneous space of dimension $\sum_{i>j}\left(m_{i, j}-1\right)+\sum_{i<j} m_{i, j}$.

Write $X(\gamma)$ for the $G_{0}$-orbit of $x_{0}$ and write $Y(\gamma)$ for its $B$-orbit. Then $X(\gamma)=$ $G_{0} / G_{0}(\gamma)$ and $Y(\gamma)=B / B(\gamma)$. Hence, 3.14 and 3.16 immediatly give a formula for the orbit dimension of $G_{0}(\gamma), \gamma \in \Gamma^{+}$.

Proposition 10. Let $\gamma=\exp _{p}\left(r_{1}, \ldots, r_{n}\right)$ be a T-point corresponding to a dominant co-character. Then the following hold.

(1) $\operatorname{dim}(X(\gamma))=-2 \sum_{i=1}^{n}(i-1) r_{i}=2 \sum_{2}^{n}(1-i) r_{i}$.

(2) Let $\mu_{r}=\exp _{p}((n-1) r,-r, \ldots,-r)$. Then $X\left(\mu_{r}\right)$ is the unique maximal dimensional orbit in $\mathbb{L a t}_{n}^{r}(K)$. It is of dimension $n(n-1) r$.

(3) The complement of $X\left(\mu_{r}\right)$ in $\mathbb{L a t}_{n}^{r}(K)$ is of codimension two.

PRoof. If $\gamma$ is dominant, then the $r_{i}$ are nonincreasing. Hence, $r_{i}-r_{j} \geq 0$ when $i<j$ and $r_{i}-r_{j} \leq 0$ otherwise. Applying (3.14), (3.16) and Lemma 13, (1), the dimension in question is $\sum_{i<j}\left(r_{i}-r_{j}\right)$. In this sum $r_{i}$ occurs with positive one coefficient $n-i$ times and with negative one coefficient $i-1$ times. This sum is $\sum_{1}^{n}(n-i) r_{i}-\sum_{1}^{n}(i-1) r_{i}=\sum_{1}^{n}(n+$ $1-2 i) r_{i}=(n+1) \sum_{1}^{n} r_{i}-2 \sum_{1}^{n} i r_{i}$. Since the $r_{i}$ sum to 0 , this is just $-2 \sum_{1}^{n} i r_{i}$. Substituting $r_{1}=\sum_{2}^{n}-r_{i}$ one obtains $-2 \sum_{2}^{n}(i-1) r_{i}=2 \sum_{2}^{n}(1-i) r_{i}$ which is the dimension formula in the statement. It is a sum with negative coefficients and $r_{i} \geq-r$ for each $i$ and so $2 \sum_{2}^{n}(1-$ $i)(-r)=n(n-1) r$ is clearly the maximum value possible and it is achieved for $\gamma=\mu_{r}$.

We show that $X\left(\mu_{r}\right)$ is the unique maximal dimensional orbit. If $\gamma \neq \mu_{r}$ then for some $i>1, r_{i}>-r$. Hence the orbit of $\gamma^{\prime}=\exp _{p}\left(r_{1}+1, \ldots, r_{i}-1, \ldots, r_{n}\right)$ is also in $\operatorname{Lat}_{n}^{r}(K)$. By direct application of (1), $\operatorname{dim} X\left(\gamma^{\prime}\right)=\operatorname{dim} X(\gamma)+n+i-2$. Hence if $\gamma \neq \mu_{r}$, there is an orbit of greater dimension. 
Since all the orbits are even dimensional, the complement of $X\left(\mu_{r}\right)$ is of codimension at least two. Let $\lambda=\exp _{p}((n-1) r-1,1-r,-r, \ldots,-r)$. Then $X(\lambda)$ is the unique orbit of codimension 2 .

Proposition 10 gives a complete description of the $G_{0}$-orbit structure of $\mathbb{L}_{a} t_{n}^{r}(K)$. As we shall see, the $B$-orbit structure is somewhat richer and more useful.

LEMMA 14. Let $\gamma=\exp _{p}\left(r_{1}, \ldots, r_{n}\right)$ and suppose that $r_{i}<r_{i+1}$. Let $\gamma^{\prime}=$ $\exp _{p}\left(r_{1}, \ldots, r_{i-1}, r_{i+1}, r_{i}, \ldots, r_{n}\right)$. Then $\operatorname{dim} Y(\gamma)+1=\operatorname{dim} Y\left(\gamma^{\prime}\right)$.

PROOF. We consider the effect of exchanging $r_{i}$ and $r_{i+1}$ in (3.16) and Lemma 13, (2). If we consider the matrix of integers, $m_{i, j}=r_{i}-r_{j}$ this causes the $i$ 'th and $(i+1)$ 'st columns to be exchanged as well as the corresponding rows. In no case is an entry moved from above the digonal to below except for the $(i, i+1)$ and $(i+1, i)$ positions which are exchanged. In the case of $\gamma, r_{i}-r_{i+1}$ is negative and hence adds nothing to the dimension of the orbit. However, $r_{i+1}-r_{i}$ being positive and subdiagonal, $r_{i+1}-r_{i}-1$ is added to the orbit dimension. In the case of $\gamma^{\prime}$, the subdiagonal element is negative, adding nothing, while the superdiagonal element is positive and so it adds $r_{i+1}-r_{i}$ to the dimension.

LEMMA 15. Let $\gamma=\exp _{p}\left(r_{1}, \ldots, r_{n}\right)$ be a T-point corresponding to a dominant cocharacter. Then, if $\gamma \neq \mu_{r}$ and $Y(\gamma) \subseteq \mathbb{L a t}_{r}^{n}(K), \operatorname{dim} Y(\gamma) \leq \operatorname{dim} \mu_{r}-2$.

PROOF. Since $\gamma$ is dominant, the $r_{i}$ are non-increasing and greater than or equal to $-r$ and since $\gamma \neq \mu_{r}, r_{1}<(n-1) r$ and there is a largest integer, $i>1$ so that $r_{i}>-r$. Let $\gamma^{\prime}=\exp _{p}\left(r_{1}+1, r_{2}, \ldots, r_{i}-1, \ldots, r_{n}\right)$. Then,$\gamma^{\prime}$ is dominant and its orbit is in $\operatorname{Lat}_{n}^{r}(K)$. Applying (35), $\operatorname{dim} Y(\gamma)+n+i-2=\operatorname{dim} Y\left(\gamma^{\prime}\right)$. Since both $n$ and $i$ are at least two, $\operatorname{dim} Y\left(\gamma^{\prime}\right) \geq \operatorname{dim} Y(\gamma)+2$.

PROPOSITION 11. The orbit, $Y\left(\mu_{r}\right)$ is the unique maximal dimensional orbit in $\mathbb{L a t}_{r}^{n}(K)$. It is affine of dimension, $n(n-1) r$ and isomorphic to affine space, $\mathbb{A}_{k}^{n(n-1) r}$. There is one unique orbit of codimension one. It is the orbit of $\delta_{r}=\exp _{p}(-r,(n-1) r,-r, \ldots,-r)$.

Proof. The dimension of $Y\left(\mu_{r}\right)$ is given by applying Lemma 13, (2) to formula (3.16). By Proposition 10, (2), its dimension is equal to the dimension of $\mathbb{L a t}_{n}^{r}(K)$ and so it is certainly of maximal dimension. It is clear that it is a surjective image of the group of upper unipotents with coefficients in $\mathcal{O}$ whence it is an affine space of the appropriate dimension. The two lemmata above show that it is unique.

Lemma 15 implies that any dominant $T$-point is of codimension at least two. Any $T$ point corresponding to a permutation of a dominant character gives an orbit of lower dimension than the dominant point by Lemma 13. Hence an orbit of codimension one can occur only as the orbit of a permutation of $\mu_{r}$. The only permutations are the points, $\exp _{p}(-r, \ldots$, $(n-1) r, \ldots,-r)$. That is, we are considering the tuple all of whose entries are $-r$ but for an $(n-1) r$ in the $i$ 'th place. By repeated applications of Lemma 14, this element has an orbit of codimension $i-1$. 
3.5. Infinitesimal families: In this section, we will compute the tangent space to a lattice in $\mathbb{L} a t_{r}^{n}$. Write $X_{r}=\mathbb{L}_{r}^{n}$ and write $\mathfrak{W}$ for the scheme of Witt vectors over $k$. Write $\mathcal{O}=\mathfrak{W}(k)$. Then $X_{r}$ represents the functor whose value on a $k$-scheme $Z$ is the set of flat families of lattices in $Z \times_{k} p^{-r} F$. If $L$ is a lattice in $X_{r}$ we shall think of it as a $\mathfrak{W}(k)=\mathcal{O}$-submodule of $p^{-r} F$ of codimension $n r$. Hence the tangent space to it consists of free $\mathfrak{W}(k[\varepsilon])$-submodules, $\tilde{L}$ of $\operatorname{Spec}(k[\varepsilon]) \times_{k} p^{-r} F=F_{r, \varepsilon}$ flat over $k[\varepsilon]$, the ring of dual numbers. For any such object let $I$ denote the ideal in the coordinate ring of $p^{-r} F$ defining $L$, and let $\tilde{I}$ denote the ideal in the coordinate ring of $F_{r, \varepsilon}$ defining $\tilde{L}$. We wish to give a complete classification of such objects.

LEMMA 16. Let $M$ be a $k$-vector space and let $k[M]$ denote the symmetric algebra on it. Assume that $\tilde{A}$ is a flat algebra of finite type over $k[\varepsilon]$ and that $\tilde{A} / \varepsilon \tilde{A} \simeq k[M]$. Then there is a natural isomorphism, $\tilde{A} \simeq k[\varepsilon][M]$. Moreover if there is an algebraic $k^{*}=G_{m, k}$-action on $M$ and $\tilde{A}$ so that the quotient map is a $k^{*}$-map, the isomorphism can be chosen to be $k^{*}$-equivariant.

Proof. The $k^{*}$-inclusion $M \hookrightarrow k[M]$ can be lifted $k^{*}$-equivariantly to a $k$-linear inclusion $M \hookrightarrow \tilde{A}$. By the universal mapping property for symmetric algebras, this extends to a map, $k[M] \rightarrow \tilde{A}$. The composite of this map with the surjection, $\tilde{A} \rightarrow k[M]$, is the identity on $M$ and so is the identity whence $k[M] \subseteq \tilde{A}$. By extension of scalars this gives a map $k[M] \otimes_{k} k[\varepsilon] \rightarrow \tilde{A}$. Let $J$ be the kernel of this map. It is clear that $J \subseteq k[M] \varepsilon$ since this is the kernel of the map to $k[M]$. On the other hand, the kernel of multiplication by $\varepsilon$ on $k[\varepsilon]$ is $k \varepsilon$ and $k \varepsilon \simeq k[\varepsilon] / \varepsilon k[\varepsilon]$. Hence tensoring the exact sequence,

$$
0 \longrightarrow k \varepsilon \longrightarrow k[\varepsilon] \stackrel{\times \varepsilon}{\longrightarrow} k[\varepsilon]
$$

with $\tilde{A}$ over $k[\varepsilon]$, by flatness, yields $\tilde{A} \varepsilon=k[M] \varepsilon$. Hence $k[M] \otimes_{k} k[\varepsilon]=k[M] \oplus k[M] \varepsilon=$ $\tilde{A}$.

The affine group scheme, $p^{-r} F / p^{(n-1) r} F$, is just $\mathbb{A}_{k}^{n^{2} r}$, affine $n^{2} r$ space. Hence $k\left[p^{-r} F / p^{(n-1) r} F\right]=k\left[x_{1,-r}, \ldots, x_{1,(n-1) r-1}, x_{2,-r}, \ldots, x_{n,(n-1) r-1}\right]$ as we observed in Lemma 7 and Proposition 6. Write it $F_{r}$. We identify the $k$-point, $x_{i, j}=a_{i, j}$ with the class of the vector $\left(u_{1}, \ldots, u_{n}\right)$ where $u_{i}$ is the class of $\sum_{j=-r}^{(n-1) r-1} \xi\left(a_{i, j}\right)^{p^{-j}} p^{j}$. Let $M=$ $\coprod_{i=-r}^{(n-1) r-1} M_{i}$ denote the graded vector space in which $M_{i}=\sum_{j=1}^{n} k x_{j, i}$ is of degree $p^{i}$. We write $k[M]$ for the coordinate ring of the affine commutative group scheme, $F_{r}$. It is the symmetric algebra on $M$ and we view $k[M]$ as graded by total weight. We recall that the closed $k^{*}$-stable subvarieties of $p^{-r} F / p^{(n-1) r} F$ are in bijective correspondence with the closed subvarieties of $\operatorname{Proj}(k[M])$ where $k[M]$ is graded by weight.

Having chosen $F$ and, if tacitly, the standard basis for it, we may define maps, $s_{i}$ : $k^{n} \rightarrow F_{r},-r \leq i<(n-1) r$. Let $\mathbf{a}=\left(a_{1}, \ldots, a_{n}\right)^{T}$ be a column vector and let $s_{i}(\mathbf{a})=$ $\sum_{j=1}^{n} \xi\left(a_{j}\right)^{p^{-i}} p^{i} e_{j} \in F_{r}$. Let $V_{i}$ denote the space of column vectors over $k$ endowed with the $k^{*}$-action, $t \circ \mathbf{a}=\left(t^{p^{i}} a_{1}, \ldots, t^{p^{i}} a_{n}\right)^{T}$. Then $s_{i}$ is a $k^{*}$-eqivariant map from $V_{i}$ to $F_{r}$ and the $\operatorname{sum}\left(\mathbf{a}_{-r}, \ldots, \mathbf{a}_{(n-1) r-1}\right) \rightarrow \sum_{i=-r}^{(n-1) r-1} s_{i}\left(\mathbf{a}_{i}\right)$ gives a $k^{*}$-isomorphism, $s: \coprod_{i=-r}^{(n-1) r-1} V_{i} \rightarrow$ 
$F_{r}$ and $x_{i, j}$ can be regarded as the $i$ 'th coordinate function on $V_{j}$ composed with the projection of $F_{r}$ on $V_{j}$.

DEFINITION 24. We shall refer to $M=\coprod_{v=-r}^{(n-1) r-1} M_{v}$ as the space of weighted linear forms on $F_{r}$. The vector subspace, $M_{s}$, will be called the space of forms of weight $p^{s}$.

In the following write $k_{\varepsilon}$ for $k[\varepsilon]$. If $Z$ is a $k$-scheme, a $k_{\varepsilon}$-flat subscheme of $Z \times_{k}$ $\operatorname{Spec}\left(k_{\varepsilon}\right)$ with special fiber, $Y \subseteq Z$, will be called an infinitesimal family of subschemes of $Z$ with special fiber $Y$. If $\tilde{Y}$ is such an infinitesimal family and it is affine, write $k_{\varepsilon}[\tilde{Y}]$ for its coordinate ring. If $Z$ is affine write $k_{\varepsilon}[Z]$ for $k[Z] \otimes_{k} k[\varepsilon]$. Finally, let $\mathcal{O}^{\varepsilon}=\mathfrak{W}(k[\varepsilon])$.

We shall give a construction for the ideals, $\tilde{I}$, defining infinitesimal families of subschemes. By the discussion above, $k[L]$ is a polynomial algebra (on a subspace of the space of weighted homogeneous forms). Let $\tilde{L}$ be a flat infinitesimal family. By Lemma 16, its coordinate ring, $k_{\varepsilon}[\tilde{L}]$ is a polynomial algebra and so $k_{\varepsilon}[\tilde{L}]=k_{\varepsilon} \otimes k[L]=k[L]+k[L] \varepsilon$. Write $\pi: k\left[F_{r}\right] \rightarrow k[L]$ for the restriction map and write $\beta: k_{\varepsilon}\left[\tilde{F}_{r}\right] \rightarrow k_{\varepsilon}[\tilde{L}]$ for restriction to $\tilde{L}$. This map has kernel $\tilde{I}$. This map is determined by its value on $k\left[F_{r}\right] \subseteq k_{\varepsilon}\left[\tilde{F}_{r}\right]$. We may write this restriction, $\beta(a)=a+\delta(a) \varepsilon$ with $\delta \in \operatorname{Der}_{k}\left(k\left[F_{r}\right], k[L]\right)$. It is classical that $\delta\left(I^{2}\right)=0$ and $\delta \mid I$ reduces to a $k[L]$-linear map from $I / I^{2}$ to $k[L]$.

We may $k^{*}$-equivariantly write $M=M_{I} \oplus M_{L}$ and so we may choose a graded algebra morphism, $\phi: k\left[M_{L}\right] \rightarrow k\left[F_{r}\right]$ splitting the restriction morphism. Finally recall that the restriction morphism for derivations $\delta \mapsto \delta \mid I$ induces an exact sequence:

$$
0 \rightarrow \operatorname{Der}_{k}(k[L], k[L]) \rightarrow \operatorname{Der}_{k}\left(k\left[F_{r}\right], k[L]\right) \rightarrow \operatorname{Hom}_{k[L]}\left(I / I^{2}, k[L]\right) \rightarrow 0
$$

and that the map $\delta \mapsto \delta \circ \phi$ gives a map from $\operatorname{Der}_{k}\left(k\left[F_{r}\right], k[L]\right)$ to $\operatorname{Der}_{k}(k[L], k[L])$ splitting the sequence (3.17). The significance of this splitting is that any $k[L]$-linear map, $\tau: I / I^{2} \rightarrow$ $k[L]$ admits an extension to a derivation, $\tilde{\tau}: k\left[F_{r}\right] \rightarrow k[L]$, that is, $\tilde{\tau}$ is a derivation whose restriction to $I$ induces $\tau$. Write $\bar{\mu}$ for the co-addition on $k[L]$ and $\mu_{N}$ for the map from $N=I / I^{2}$ to $N \otimes_{k} k[L] \oplus k[L] \otimes_{k} N$.

Proposition 12. Each $\delta \in \operatorname{Der}_{k}\left(k\left[F_{r}\right], k[L]\right)$ determines a unique ideal $\tilde{I}_{\delta} \subseteq k_{\varepsilon}\left[\tilde{F}_{r}\right]$ so that the quotient, $k_{\varepsilon}\left[\tilde{F}_{r}\right] / \tilde{I}_{\delta}=k_{\varepsilon}[\tilde{L}]$ is the coordinate ring of an infinitesimal family of subschemes with special fiber, $L$. The ideal, $\tilde{I}_{\delta}$ is given by the formula, $\tilde{I}_{\delta}=\{x+y \varepsilon \mid \delta(x) \equiv$ $-y(\bmod I)\}$. Two such derivations, $\delta$ and $\delta^{\prime}$ determine the same ideal if and only if $\delta \mid I=$ $\delta^{\prime} \mid I$.

PROOF. Let $\bar{a}, \bar{b}$, etc. denote the restriction of $a, b \in k\left[F_{r}\right]$ to $L$. Given $\delta$ define $\beta$ : $k_{\varepsilon}\left[\tilde{F}_{r}\right] \rightarrow k_{\varepsilon}[\tilde{L}]$ by the equation $\beta(a)=\bar{a}+\delta(a) \varepsilon$. Then $\beta$ extends to $k_{\varepsilon}[\tilde{L}]$ by the equation, $\beta(a+b \varepsilon)=\bar{a}+(\bar{b}+\delta(a)) \varepsilon$. Now $\beta$ is a surjection onto $k_{\varepsilon}[\tilde{L}]$ and so it defines an ideal, $\tilde{I}_{\delta}=\operatorname{ker}(\beta)$. (Notice that this definition of $\beta$ is consistent with the definition given in the discussion preceding (3.17).) If $\delta^{\prime}=\delta+\gamma$ where $\gamma$ is a derivation vanishing on $I$, let $\bar{\gamma}$ denote the element of $\operatorname{Der}_{k}(k[L], k[L])$ induced by $\gamma$. Let $\beta^{\prime}$ be the surjection corresponding to $\delta^{\prime}$ and let $\psi: k_{\varepsilon}[\tilde{L}] \rightarrow k_{\varepsilon}[\tilde{L}]$ be the automorphism defined by $\psi(\bar{a}+\bar{b} \varepsilon)=\bar{a}+(\bar{b}+\bar{\gamma}(\bar{a})) \varepsilon$. Clearly, $\beta^{\prime}=\psi \circ \beta$. Hence if $\delta^{\prime}=\delta+\gamma, \operatorname{ker}(\beta)=\operatorname{ker}\left(\beta^{\prime}\right)$. Conversely suppose that 
$\tilde{I}_{\delta}=\tilde{I}_{\delta^{\prime}}=\tilde{I}$. Then there are isomorphisms $\bar{\beta}: k_{\varepsilon}\left[\tilde{F}_{r}\right] / \tilde{I} \rightarrow k_{\varepsilon}[\tilde{L}]$ and $\bar{\beta}^{\prime}$ defined similarly. Let $\psi=\bar{\beta}^{-1} \circ \bar{\beta}$. Then $\psi(\bar{a})=\bar{a}+\bar{\gamma}(\bar{a}) \varepsilon$, and if $\gamma \in \operatorname{Der}_{k}\left(k\left[F_{r}\right], k[L]\right)$ is a derivation inducing $\bar{\gamma}, \delta^{\prime}=\delta+\gamma$. The formula for $\tilde{I}_{\delta}$ is clear.

By Proposition 12, ideals defining infinitesimal families of subschemes of $\tilde{F}_{r}$ with special fiber $L$ correspond to elements of $\operatorname{Hom}_{k[L]}(N, k[L])$. We wish to examine the condition which corresponds to the requirement that $\tilde{I}_{\delta}$ define an infinitesimal family of subgroup shecmes. If $\delta \in \operatorname{Hom}_{k[L]}(N, k[L])$ is a co-normal vector we shall say that it is co-additive if ( $\delta \otimes$ id, id $\otimes \delta) \circ \mu_{N}=\bar{\mu} \circ \delta$. (our convention here is $(\delta \otimes$ id, id $\otimes \delta)(u \otimes a, b \otimes v)=\delta(u) \otimes a+$ $b \otimes \delta(v))$

To better understand the significance of co-additivity recall that if $H \subseteq G$ is a closed group subscheme of $G, \mathcal{I}$ is the sheaf of ideals defining $H$ and $j$ is the embedding, then $j^{*} \mathcal{I}=\mathcal{I} / \mathcal{I}^{2}$ is the normal bundle to $H$ in $G$. Write it $\mathcal{N}_{G / H}$.

Proposition 13. Let $G=\operatorname{Spec}(A)$ be an affine commutative smooth group scheme over $k$ and let $H=\operatorname{Spec}(A / I)$ be a closed smooth subgroup scheme. Let $\mu: A \rightarrow A \otimes_{k} A$ be the co-multiplication and write $B=A / I$. Let $J=A \otimes I+I \otimes A \subseteq A \otimes A$. Then the following hold.

(1) Let $N_{G / H}=\Gamma\left(H, \mathcal{N}_{G / H}\right)$. Then $N_{G / H}$ admits two rational $H$-actions. Under either, it is free and it admits a basis of invariant sections canonically isomorphic to $(I+$ $\left.\mathfrak{m}^{2}\right) / \mathfrak{m}^{2}$, the fiber at the origin.

(2) There is a canonical isomorphism, $J / J^{2} \rightarrow\left(B \otimes N_{G / H} \oplus N_{G / H} \otimes B\right)$.

(3) There is a natural mapping, $\mu_{N}: N_{G / H} \rightarrow N_{G / H} \otimes_{k} B \oplus B \otimes_{k} N_{G / H}$ induced by $\mu$ so that if $x$ is an invariant section, $\mu_{N}(x)=(x \otimes 1,1 \otimes x)$.

Proof. The module $N_{G / H}$ is just $I / I^{2}$. Let $J=A \otimes I+I \otimes A$. Since $I$ defines a subgroup scheme, $\mu(I) \subseteq I \otimes A+A \otimes I$ and so $\mu\left(I^{2}\right) \subseteq\left(A \otimes I^{2}+I \otimes I+I^{2} \otimes A\right)=J^{2}$ and so $\mu$ induces a map, $\bar{\mu}: I / I^{2} \rightarrow J / J^{2}$.

The vector spaces, $I \otimes I$ and $I^{2} \otimes A$ are both subspaces of $I \otimes A$ and so $J^{2} \subseteq I^{2} \otimes A+$ $A \otimes I$. Hence there is a surjective map, $q_{1}: J / J^{2} \rightarrow(I \otimes A+A \otimes I) /\left(I^{2} \otimes A+A \otimes I\right)$. Applying the first isomorphism theorem of group theory and keeping track of intersections it is clear that $q_{1}$ maps $J / J^{2}$ to $I / I^{2} \otimes A / I$. In particular $q_{1} \circ \bar{\mu}$ maps $I / I^{2}$ to $I / I^{2} \otimes A / I$.

This is a co-action of $A / I=k[H]$ on $N_{G / H}=I / I^{2}$ making it a homogeneous bundle on $H$. Write $B$ for $A / I$. Homogeniety implies that $I / I^{2}$ contains an $H$-invariant basis isomorphic to the fiber at the origin which is isomorphic to $I /\left(I \cap \mathfrak{m}^{2}\right)=\left(I+\mathfrak{m}^{2}\right) / \mathfrak{m}^{2}$. This establishes (1) for the right co-action, $q_{1} \circ \bar{\mu}$.

The observations above can be applied to left rather than right translation. By an entirely symmetric argument there is a projection, $q_{2}$, from $J / J^{2}$ to $A \otimes I /\left(A \otimes I^{2}+I \otimes I\right)=$ $A / I \otimes I / I^{2}$. This map must be twisted by the antipode if it is to agree with the usual notion of left translation but $G$ is commutative. Hence the twist may be omitted. Thus there is a map $q_{2}: J / J^{2} \rightarrow A / I \otimes I / I^{2}$ so that $q_{2} \circ \bar{\mu}: I / I^{2} \rightarrow A / I \otimes I / I^{2}$ is a bundle co-action corresponding to left translation. Thus $q_{2} \circ \bar{\mu}$ is a second co-action homogenizing the normal 
bundle. These are the two actions on the normal bundle. Since these actions make the normal bundle into a homogeneous bundle on the group $H$, the first assertion is established.

Let $\pi_{1}, \pi_{2}$ denote the projections $G \times G \rightarrow G$. Then it is commonplace to note that over a flat base, $\mathcal{N}_{G \times G / H \times H}=\pi_{1}^{*} \mathcal{N}_{G / H} \oplus \pi_{2}^{*} \mathcal{N}_{G / H}$. On global sections this isomorphism is realized as the product map, $\left(q_{1}, q_{2}\right): J / J^{2} \rightarrow B \otimes N_{G / H} \oplus N_{G / H} \otimes B$. This establishes the second assertion.

If $\bar{\mu}$ is the map from $I / I^{2}$ to $J / J^{2}$, then $q_{1} \circ \bar{\mu}$ and $q_{2} \circ \bar{\mu}$ are left and right co-actions on $N_{G / H}$ and it is clear that they have the same invariants. If $x$ is such an invariant section, then $q_{1} \circ \bar{\mu}(x)=1 \otimes x$ and $q_{2} \circ \bar{\mu}(x)=x \otimes 1$. Let $\mu_{N}=\left(q_{1} \circ \mu_{0}, q_{2} \circ \mu_{0}\right)$. The third statement is now clear.

COROllary 2. Let $\mathfrak{m}$ be the ideal defining 0 in $F_{r}$. Let $M_{I}=M \cap\left(I+\mathfrak{m}^{2}\right)$. The natural map from $M_{I}$ to $I / I^{2}$ carries $M_{I}$ to the $L$-invariants of $I / I^{2}=N_{F_{r}} / L$. The module $I / I^{2}$ is $k^{*}$-isomorphic to $M_{I} \otimes_{k} k[L]$ and, for $u \in M_{I}$ the map $\mu_{I}$ is determined by the formula $\mu_{I}(u \otimes 1)=(u \otimes 1) \otimes 1+1 \otimes(u \otimes 1)$.

PROOF. First note that the coordinate ring, $k\left[F_{r}\right]=k[M]$, is the symmetric algebra on the space of weighted linear forms. Furthermore if $\mathfrak{m}$ is the ideal defining the origin, $M \subseteq \mathfrak{m}$ and the composite of this inclusion with the natural surjection is a $k^{*}$-equivariant isomorphism, $j: M \rightarrow \mathfrak{m} / \mathfrak{m}^{2}$.

Write $\overline{\mathfrak{m}}$ for the ideal defining the identity in $k[L]$. Then

$$
0 \rightarrow\left(I+\mathfrak{m}^{2}\right) / \mathfrak{m}^{2} \rightarrow \mathfrak{m} / \mathfrak{m}^{2} \rightarrow \overline{\mathfrak{m}} / \overline{\mathfrak{m}}^{2} \rightarrow 0
$$

is exact. Now $I / I^{2}=N_{F_{r} / L}$ is homogeneous by Proposition 13 and so it is freely generated by its $L$-invariants which as a $k$-vector space are isomorphic to $\left(I / I^{2}\right) / \overline{\mathfrak{m}}\left(I / I^{2}\right)=(I+$ $\left.\mathfrak{m}^{2}\right) / \mathfrak{m}^{2}$ by the proof of Proposition $13,(1)$. Clearly $j^{-1}\left(\left(I+\mathfrak{m}^{2}\right) / \mathfrak{m}^{2}\right)=M_{I}$. Thus $j$ is a $k^{*}$ isomorphism from $M_{I}$ to the fiber of $I / I^{2}$ at the origen. The natural projection $I / I^{2} \rightarrow(I+$ $\left.\mathfrak{m}^{2}\right) / \mathfrak{m}^{2}$ induces a $k^{*}$-isomorphism from $\left(I / I^{2}\right)^{L}$ to $j\left(M_{I}\right)$. Composing $j$ with the inverse of this isomorphism we obtain a $k^{*}$-isomorphism, $\tilde{j}: M_{I} \rightarrow\left(I / I^{2}\right)^{L}$. Identifying $M_{I}$ with its image under $\tilde{j}$ (this does not respect co-multiplication), we may write $N=M_{I} \otimes_{k} k[L]$. The lemma is now nothing more than Proposition 13, (3) subject to the identification of $M_{I} \otimes 1$ with the $L$-invariants in $N$.

We recall thet if $H=\operatorname{Spec} C$ is an affine group scheme with co-multiplication, $\mu$ : $C \rightarrow C \otimes C$, then an additive character of $H$ is just a morphism of algebraic groups from $H$ to $G_{a, k}$. The additive characters of $H$ are exactly the functions $f \in H$ such that $\mu(f)=$ $f \otimes 1+1 \otimes f$. Notice that, over a field of positive characteristic, if $F(x)=\sum_{i} a_{i} x^{p^{i}}$ is an additive polynomial and $f$ is an additive character, then $F(f)$ is also an additive character. Write $\mathcal{A}(H)$ for the group of additive characters of $H$.

If $\delta \in \operatorname{Hom}_{k[L]}\left(I / I^{2}, k[L]\right)$ is a co-normal vector, we shall say that it is co-additive if $\bar{\mu} \circ \delta=(\pi \otimes \delta+\delta \otimes \pi) \circ \mu$. 
COROLLARY 3. Let $\delta$ be a co-additive co-normal vector in $\operatorname{Hom}_{k[L]}\left(I / I^{2}, k[L]\right)$. Then $\delta \mid\left(I / I^{2}\right)$ is a linear map from $M_{I}$ to $\mathcal{A}(H)$, the group of additive characters of $L$.

Proof. The co-normal vector $\delta$ induces a $k[L]$-map from $I / I^{2}$ to $k[L]$. By Proposition 13 and Corollary $2, I / I^{2} \simeq M_{I} \otimes k[L]$ and the isomorphism is a morphism of homogeneous bundles. Hence, $\delta$ is uniquely determined by its restriction to $M_{I}$ the submodule of invariant sections. If $u \in M_{I}$ then $q_{1} \circ \bar{\mu}(u)=u \otimes 1$ and $q_{2} \circ \bar{\mu}(u)=1 \otimes u$ by invariance and the fact that these are the left and right translation co-actions.

If $\eta, \zeta: I / I^{2} \rightarrow k[L]$ are two maps, $(\eta \otimes \pi+\pi \otimes \zeta) \circ \mu(u)=(\eta \otimes \mathrm{id}, \mathrm{id} \otimes \zeta) \circ \mu_{N}(u)$. In particular, by the observation we made just above, if $\delta$ is co-additive and $u \in M_{I}$, then the equation, $\bar{\mu}(\delta(u))=(\pi \otimes \delta+\delta \otimes \pi) \circ \mu(u)=(\mathrm{id} \times \delta, \delta \otimes \mathrm{id}) \circ\left(q_{1} \circ \bar{\mu}(u) q_{2} \circ \bar{\mu}(u)\right)=$ $(\mathrm{id} \otimes \delta, \delta \otimes \mathrm{id})(1 \otimes u, u \otimes 1)=(1 \otimes \delta(u)+\delta(u) \otimes 1)$. This is exactly the equation which says that $\delta(u)$ is an additive character of $L$. Thus, for each invariant, $u \in M_{I}, \delta(u)$ is an additive character.

Proposition 14. Suppose that $\delta \in \operatorname{Hom}_{k[L]}(N, k[L])$. Let $\tilde{\delta}$ be a derivation of $k\left[F_{r}\right]$ in $k[L]$ restricting to $\delta$. Then $\tilde{I}_{\delta}$ defines an additive $k_{\varepsilon}$-subgroup scheme of $\tilde{F}_{r}$ if and only if $\delta$ is co-additive. When $\delta$ is co-additive, let $\left.\lambda^{0}=(\tilde{\delta} \circ \phi \otimes \mathrm{id}+\mathrm{id} \otimes \tilde{\delta} \circ \phi) \circ \mu-\bar{\mu} \circ \tilde{\delta}\right)$. Then $\lambda^{0}$ is a derivation of $k\left[\tilde{F}_{r}\right]$ in $k[L] \otimes k[L]$ vanishing on I. If $\lambda: k[L] \rightarrow k[L] \otimes k[L]$ is the induced derivation, the map $\mu_{\delta}(a+b \varepsilon)=\bar{\mu}(a)+(\bar{\mu}(b)+\lambda(a)) \varepsilon$ is a co-multiplication making $\tilde{L}$ an infinitesimal family of subgroups.

Proof. Let $\beta$ be the map associated to $\tilde{\delta}$ as in the proof of Proposition 12. Just write $\mu$ for the co-multiplication on $k_{\varepsilon}\left[\tilde{F}_{r}\right]$. Then $\tilde{I}_{\delta}$ defines a subgroup scheme if an only if $\beta \otimes \beta$ 。 $\mu\left(\tilde{I}_{\delta}\right)=(0)$, in which case the co-multiplication on $k_{\varepsilon}[\tilde{L}]$ is just the quotient map induced by $\mu$. That is, consider the diagram:

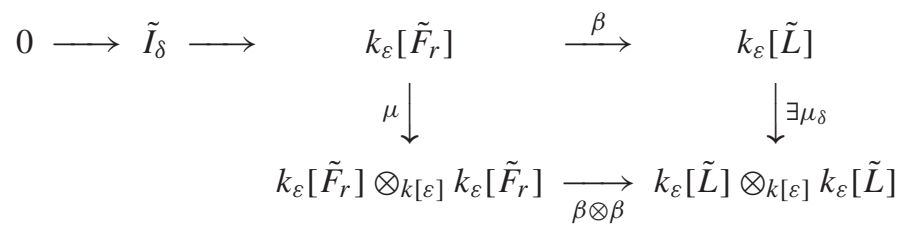

Then $\tilde{I}_{\delta}$ defines a subgroup scheme if and only if the map $\mu_{\delta}$ exists rendering the diagram commutative.

Consider $\beta \otimes \beta$ on an element of the form $a \otimes b$. Then $(\beta \otimes \beta)(a \otimes b)=\beta(a) \otimes \beta(b)=$ $(\pi(a)+\tilde{\delta}(a) \varepsilon) \otimes(\pi(b)+\tilde{\delta}(b) \varepsilon)=\pi(a) \otimes \pi(b)+(\pi(a) \otimes \tilde{\delta}(b)+\tilde{\delta}(a) \otimes \pi(b)) \varepsilon$. That is, $\beta \otimes \beta(u)=\pi \otimes \pi(u)+(\tilde{\delta} \otimes \pi+\pi \otimes \tilde{\delta})(u) \varepsilon$ for $u \in k\left[F_{r}\right] \otimes k\left[F_{r}\right]$.

A necessary and sufficient condition for the existence of $\mu_{\delta}$ is that $(\beta \otimes \beta) \circ \mu\left(\tilde{I}_{\delta}\right)=(0)$. Elements of the form $a-\phi \circ \tilde{\delta}(a) \varepsilon, a \in I$ generate $\tilde{I}_{\delta}$ and so it is necessary and sufficient that $(\beta \otimes \beta) \circ \mu$ should vanish on such elements. But $(\beta \otimes \beta) \circ \mu(a-\phi \circ \tilde{\delta}(a) \varepsilon)=(\pi \otimes \pi) \circ \mu(a)+$ $\{(\pi \otimes \tilde{\delta}+\tilde{\delta} \otimes \pi) \circ \mu(a)-(\pi \otimes \pi) \circ \mu(\phi \circ \tilde{\delta}(a)\} \varepsilon$. Since $a \in I$ and $(\pi \otimes \pi) \circ \mu \circ \phi=\bar{\mu} \circ \pi \circ \phi=\bar{\mu}$ we may continue $(\beta \otimes \beta) \circ \mu(a-\phi \circ \tilde{\delta}(a) \varepsilon)=\{(\pi \otimes \tilde{\delta}+\tilde{\delta} \otimes \pi) \circ \mu-\bar{\mu} \circ \tilde{\delta}\}(a) \varepsilon$. The 
vanishing of this expression in $I$ is exactly the co-additivity of $\delta$. Thus it is clear that $\mu_{\delta}$ can be defined if and only if $\delta$ is co-additive.

We may now compute a formula for $\mu_{\delta}$. If $\bar{a}+\bar{b} \varepsilon \in k_{\varepsilon}[\tilde{L}]$ choose $a$ and $b$ in $k\left[F_{r}\right]$ so that $\pi(a)=\bar{a}, \pi(b)=\bar{b}$. Then $\bar{a}+\bar{b} \varepsilon=\beta(a+(b-\phi \circ \tilde{\delta}(a)) \varepsilon)$. Then $(\beta \otimes \beta) \circ \mu(a+(b-\phi \circ$ $\tilde{\delta}(a)) \varepsilon)=(\pi \otimes \pi) \circ \mu(a)+\{(\pi \otimes \tilde{\delta}+\tilde{\delta} \otimes \pi) \circ \mu(a)+(\pi \otimes \pi) \circ \mu(b)-(\pi \otimes \pi) \circ \mu(\phi \circ \tilde{\delta}(a))\} \varepsilon$. Recalling that $(\pi \otimes \pi) \circ \mu=\bar{\mu} \circ \pi, \pi(a)=\bar{a}, \pi(b)=\bar{b}$ and that $\pi \circ \phi=$ id this shows that $\mu_{\delta}(\bar{a}+\bar{b} \varepsilon)=(\beta \otimes \beta) \circ \mu(a+(b-\phi \circ \tilde{\delta}(a)) \varepsilon)=\bar{\mu}(\bar{a})+\{\bar{\mu}(\bar{b})+((\pi \otimes \tilde{\delta}+\tilde{\delta} \otimes \pi) \circ \mu-$ $\bar{\mu} \circ \tilde{\delta})(a)\} \varepsilon=\bar{\mu}(\bar{a})+(\bar{\mu}(\bar{b})+\lambda(\bar{a})) \varepsilon$.

These results give a good description of the infinitesimal deformations of a lattice in $p^{-r} F$ of codimension $n r$, but what has been described is the deformations of a lattice in the functor corresponding to families of subgroups. The contravariant description in terms of the coordinate ring makes it quite awkward to characterize those infinitesimal families which are families of lattices. For that a covariant description in terms of points would be more natural. This requires some generalities concerning ring schemes. Let $\mathfrak{R}$ be a commutative unitary ring scheme over $k$ and let $\mathfrak{M}$ be a scheme of modules over $\mathfrak{R}$. Let $\mathcal{O}=\mathfrak{R}(k)$ and let $M=\mathfrak{M}(k)$. Let $\mathfrak{R}=\operatorname{Spec}(R)$ and let $\mathfrak{M}=\operatorname{Spec}(A)$. As we must negotiate a blizzard of notation, and the multiplication and addition are commutative, we shall not be fastidious about such matters as co-associativitity and left and right translation. We adopt the following notation:

Notation. (1) $\alpha: R \rightarrow R \otimes_{k} R$ and $\beta: A \rightarrow A \otimes_{k} A$ are the two co-additions.

(2) $\quad \eta: R \rightarrow R \otimes_{k} R$ is co-multiplication and $\varsigma: A \rightarrow R \otimes_{k} A$ is the scalar co-action.

(3) $m_{A}: A \otimes A \rightarrow A$ and $m_{R}: R \otimes R \rightarrow R$ are the multiplications, $a \otimes b \mapsto a b$ and $\tau: P \otimes Q \rightarrow Q \otimes P$ is reversal of factors.

(4) $e_{0}: R \rightarrow k, e_{1}: R \rightarrow k$ and $e_{0}^{\prime}: A \rightarrow k$ are evaluation at 0 and 1 in $\mathcal{O}$ and at 0 in $M$, respectively.

(5) $D_{\mathcal{O}}$ and $D_{M}$ denote the tangent spaces of $\mathfrak{R}$ and $\mathfrak{M}$ at their zero's. They are the Lie algebras of these schemes regarded as additive commutative group schemes.

(6) $R^{*}$ and $A^{*}$ denote the linear duals of $R$ and $A$, respectively.

(7) $e_{0}$ and $e_{0}^{\prime}$ represent the homomorphisms, evaluation at 0 in $\mathfrak{R}$ and $\mathfrak{M}$, respectively and $\mathfrak{m}_{R}=\operatorname{ker}\left(e_{0}\right)$ and $\mathfrak{m}_{A}=\operatorname{ker}\left(e_{0}^{\prime}\right)$.

Having introduced this notation we now define the following operations:

$$
\begin{aligned}
& u \diamond v=(u \otimes v) \circ \alpha, \quad u, v \in R^{*} \quad \text { (additive convolution) } \\
& a \diamond b=(a \otimes b) \circ \beta, \quad a, b \in A^{*} \quad \text { (same) } \\
& u \star v=(u \otimes v) \circ \eta, \quad u, v \in R^{*} \quad \text { (multiplicative convolution) } \\
& u \star a=(u \otimes a) \circ \varsigma, \quad u \in R^{*}, a \in A^{*} \quad \text { (scalar convolution) }
\end{aligned}
$$

These operations are all associative and they distribute over ordinary addition. That is $u \diamond(v+w)=u \diamond v+u \diamond w, u \star(v+w)=u \star v+u \star w$ etc. Notice here that + denotes ordinary pointwise sum in the two linear duals, $R^{*}$ and $A^{*}$. The addition in $\mathcal{O}$ and in $M$ will be, 
for the duration of this discussion, written as $\diamond$. However there is a subtlety regarding distributivity of multiplicative convolution over additive convolution. To explain this we consider the diagram describing the distributivity condition, $u \star(a \diamond b)=(u \star a) \diamond(u \star b), u \in \mathcal{O}$, $a, b \in M$.

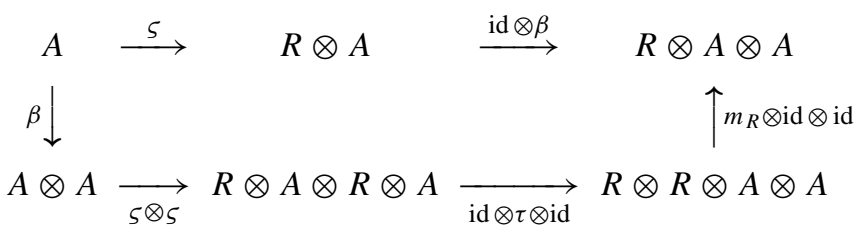

The second distributivity condition, $(u \diamond v) \star a=(u \star a) \diamond(v \star a)$, is encoded in the diagram:

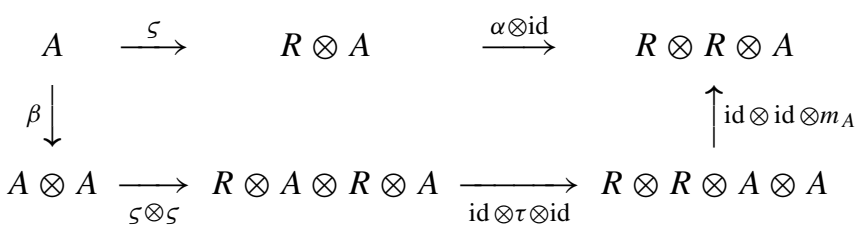

For $u \in R^{*}, a, b \in A^{*}$ we wish to establish that $u \star(a \diamond b)=(u \star a) \diamond(u \star b)$. Let $\gamma=(\mathrm{id} \otimes \tau \otimes \mathrm{id}) \circ(\varsigma \otimes \varsigma) \circ \beta$. Then (3.24) yields the equation, $(u \otimes a \otimes b) \circ\left(m_{R} \otimes \mathrm{id} \otimes \mathrm{id}\right) \circ \gamma=$ $(u \otimes a \otimes b) \circ(\mathrm{id} \otimes \beta) \circ \varsigma$. This is actually a distributivity equation only if $u \circ m_{R}=u \otimes u$, that is, only if $u$ is a homomorphism, that is to say an element of $\mathcal{O}$. Thus the distributive rules which hold are these:

$$
\begin{array}{ll}
(u \diamond v) \star x=(u \star x) \diamond(v \star x), & u, v \in R^{*}, x \in \mathcal{O} \\
x \star(a \diamond b)=(x \star a) \diamond(x \star b), & a, b \in A^{*}, x \in \mathcal{O} \\
(u \diamond v) \star q=(u \star q) \diamond(v \star q), & u, v \in R^{*}, q \in M
\end{array}
$$

Our aim is to understand the ring, $\mathfrak{R}(k[\varepsilon])=\mathcal{O}^{\varepsilon}$ and the module, $\mathfrak{M}(k[\varepsilon])=M^{\varepsilon}$. To this end, for $u \in \mathcal{O}, a \in M$ let $t_{u}$ (respectively $t_{a}$ ) denote translation by $u$ (respectively $a$ ). That is, $t_{u}(f)(v)=f(u \diamond v), t_{a}(g)(b)=g(a \diamond b)$. Then if $\delta \in D_{\mathcal{O}}, \gamma \in D_{M}$ by well known calculations, $\delta \circ t_{u}=\delta \diamond u$ and $\gamma \circ t_{a}=\gamma \diamond a$. We shall write $\delta_{u}$ for $\delta \circ t_{u}$. Here $\delta$ and $\gamma$ are tangent vectors at 0 on $R$ and $A$ respectively. That is, $\delta(f g)=e_{0}(f) \delta(g)+e_{0}(g) \delta(f)$ and the same for $\gamma$. Now $\mathcal{O}^{\varepsilon}$ is by definition the set of pairs $(u, \lambda)$ where $u \in \mathcal{O}$ and $\lambda$ is a tangent vector at $u$. The pair $(u, \lambda)$ corresponds to the homomorphism $(u, \lambda)(f)=f(u)+\lambda(f) \varepsilon$. The same holds for $M$.

Now $\lambda$ can always be written uniquely as $\delta_{u}$ for some $\delta \in D_{\mathcal{O}}$. The same reasoning applies to $M^{\varepsilon}$. Hence $\mathcal{O}^{\varepsilon}=\left\{\left(u, \delta_{u}\right): u \in \mathcal{O}, \delta \in D_{\mathcal{O}}\right\}$ and $M^{\varepsilon}=\left\{\left(a, \gamma_{a}\right): a \in M, \gamma \in\right.$ $\left.D_{M}\right\}$. 
LEMMA 17. The ring $\mathfrak{R}(k[\varepsilon])=\mathcal{O}^{\varepsilon}$ is the set of pairs $\left\{\left(u, \delta_{u}\right): u \in \mathcal{O}, \delta \in D_{\mathcal{O}}\right\}$ and $\mathfrak{M}(k[\varepsilon])=M^{\varepsilon}=\left\{\left(a, \gamma_{a}\right): a \in M, \gamma \in D_{M}\right\}$. The formulae for addition are:

$$
\begin{aligned}
& \left(u, \delta_{u}\right) \diamond\left(v, \theta_{v}\right)=\left(u \diamond v, \delta_{u} \diamond v+\theta_{v} \diamond u\right)=\left(u \diamond v, \delta_{u \diamond v}+\theta_{u \diamond v}\right), \\
& \left(a, \gamma_{a}\right) \diamond\left(b, v_{b}\right)=\left(a \diamond b, \gamma_{a \diamond b}+v_{a \diamond b}\right) .
\end{aligned}
$$

The formulae for multiplication are:

$$
\begin{aligned}
& \left(u, \delta_{u}\right) \star\left(v, \gamma_{v}\right)=\left(u \star v,(\delta \star v)_{u \star v}+(\gamma \star u)_{u \star v}\right), \\
& \left(u, \delta_{u}\right) \star\left(a, \lambda_{a}\right)=\left(u \star a,(u \star \lambda)_{u \star a}+(\delta \star a)_{u \star a}\right) .
\end{aligned}
$$

The subset $\left\{(0, \delta): \delta \in D_{\mathcal{O}}\right\}$ is an ideal of square zero in $\mathcal{O}^{\varepsilon}$ and it annihilates the submodule $\left\{(0, \gamma): \gamma \in D_{M}\right\}$ in $M^{\varepsilon}$.

PROOF. We shall establish the formulae for addition in $M^{\varepsilon}$ and for the corresponding scalar multiplication. The other formulae are a special case. Let $(\phi, \delta): B \rightarrow k[\varepsilon]$ and $(\psi, \gamma): C \rightarrow k[\varepsilon]$ be two homomorphisms. That is, $(\phi, \delta)(b)=\phi(b)+\delta(b) \varepsilon$ and the same for $(\psi, \gamma)$. Then $[(\phi, \delta) \otimes(\psi, \gamma)](b \otimes c)=(\phi(b)+\delta(b) \varepsilon)(\psi(c)+\gamma(c) \varepsilon)=\phi(b) \psi(c)+$ $(\phi(b) \gamma(c)+\delta(b) \psi(c)) \varepsilon=(\phi \otimes \psi, \phi \otimes \gamma+\delta \otimes \psi)(b \otimes c)$. That is, $(\phi, \delta) \otimes(\psi, \gamma)=$ $(\phi \otimes \psi, \phi \otimes \gamma+\delta \otimes \psi)$. Consequently, $\left(a, \gamma_{a}\right) \diamond\left(b, \eta_{b}\right)=\left(\left(a, \gamma_{a}\right) \otimes\left(b, \eta_{b}\right)\right) \circ \alpha=$ $\left(a \otimes b, a \otimes \eta_{b}+\gamma_{a} \otimes b\right) \circ \alpha=\left(a \otimes b \circ \alpha,\left(a \otimes \eta_{b}+\gamma_{a} \otimes b\right) \circ \alpha\right)=\left(a \diamond b, a \diamond \eta_{b}+\gamma_{a} \diamond b\right)$. By the commutativity and associativity of additive convolution, $a \diamond \eta_{b}=a \diamond(\eta \diamond b)=\eta \diamond(a \diamond b)=\eta_{a \diamond b}$ and the same for $\gamma_{a} \diamond b$. This permits us to continue our calculation. The desired expression is $\left(a \diamond b, \eta_{a \diamond b}+\gamma_{a \diamond b}\right)$. This establishes (3.28).

Before proceeding with scalar multiplication an observation is necessary. For $u \in$ $\mathcal{O}, f \in R, g \in A$, define $c_{u}$ by the equations, $c_{u}(f)(v)=f(u \star v), c_{u}(g)(a)=g(u \star a)$. Then since multiplication by $u$ is an additive endomorphism of commutative group schemes, $c_{u}$ fixes the ideals $\mathfrak{m}_{R}$ and $\mathfrak{m}_{A}$ and induces an endomorphism of the Lie algebras, $D_{\mathcal{O}}$ and $D_{M}$ (indeed of restricted Lie algebras for $k$ of positive characteristic). Then for $\delta \in D_{\mathcal{O}}, \gamma \in D_{M}$, as was the case for additive convolution, $\delta \circ c_{u}=\delta \star u$ and $\gamma \circ c_{u}=\gamma \star u$. If $u \in \mathcal{O}, \gamma \in D_{M}$, 53 permits us to write $u \star \gamma_{a}=u \star(\gamma \diamond a)=(u \star \gamma) \diamond(u \star a)=(u \star \gamma)_{u \star a}$. A similar equation holds for $\left(\delta_{u}\right) \star a$.

To establish (3.29), we proceed as we did for (3.28) except for the last step, when the computation of the second component of $\left(u, \delta_{u}\right) \star\left(a, \gamma_{a}\right)$ requires an application of the distributivity equations, (3.25), noting that $u \in \mathcal{O}$ and $a \in M$. This second component becomes $u \star \gamma_{a}+\delta_{u} \star a=(u \star \gamma)_{u \star a}+(\delta \star a)_{u \star a}$. Thus both pairs of formulae are proven and then the last two assertions follow easily from these formulae.

Corollary 4. Let $\lambda$ be in $D_{M}$ and let $u, v \in \mathcal{O}$. Then $\lambda \star(u \diamond v)=(\lambda \star u)+(\lambda \star v)$. Hence the $k$-vector spaces, $D_{M}$ and $D_{\mathcal{O}}$, are $\mathcal{O}$-modules under multiplicative convolution.

Proof. Identify $D_{M}$ with the submodule of $M^{\varepsilon},\left\{(0, \gamma): \gamma \in D_{M}\right\}$ and identify $D_{\mathcal{O}}$ with the corresponding square zero ideal. Then $D_{M}$ is a $\mathcal{O}^{\varepsilon}$-submodule of $M^{\varepsilon}$ annihilated by $D_{\mathcal{O}}$. The corollary follows at once. 
DEFinition 25. Let $\mathfrak{R}$ be a $k$-scheme of rings and let $\mathfrak{M}$ be a scheme of $\mathfrak{R}$-modules. Then we shall refer to the tangent space to $\mathfrak{M}$ at 0 , thought of as a $\mathfrak{R}(k)$-module as its infinitesimal module. We shall write it as $D(\mathfrak{M})$.

Having completed the most delicate of our computations, we revert to a simpler notation. If $\mathcal{O}=\mathfrak{R}(k)$ we shall use + rather than $\diamond$ for the addition and simple juxtaposition for the product. We can summarize our computations in the following, which does nothing but restate Lemma 17 in simpler notation.

PROposition 15. Let $\mathfrak{R}$ be a scheme of rings and let $\mathfrak{M}$ be a scheme of $\mathfrak{R}$-modules. Let $\mathcal{O}=\mathfrak{R}(k), M=\mathfrak{M}(k)$. Write $\mathcal{O}^{\varepsilon}$ and $M^{\varepsilon}$ for their groups of points in $k[\varepsilon]$. There is a short exact sequence,

$$
0 \rightarrow D(\mathfrak{M}) \rightarrow M^{\varepsilon} \rightarrow M \rightarrow 0
$$

As an $\mathcal{O}^{\varepsilon}$-module $D(\mathfrak{M})$ is annihilated by $D(\mathfrak{R})$. Furthermore, $\mathcal{O}^{\varepsilon}=\left\{\left(u, \delta_{u}\right): u \in \mathcal{O}, \delta \in\right.$ $D(\mathfrak{R})\}$ and $M^{\varepsilon}=\left\{\left(a, \lambda_{a}\right): a \in M \lambda \in D(\mathfrak{M})\right\}$. Addition and scalar multiplication are given by the formulae:

$$
\begin{aligned}
\left(a, \lambda_{a}\right)+\left(b, v_{b}\right) & =\left(a+b, \lambda_{a+b}+v_{a+b}\right), \\
\left(u, \delta_{u}\right)\left(a, \lambda_{a}\right) & =\left(u a, u \lambda_{u a}+\delta a_{u a}\right) .
\end{aligned}
$$

The ideal $D(\mathfrak{R})$ is of square zero and it annihilates $D(\mathfrak{M})$.

We shall use this description to complete our examination of the tangent space to a lattice in $\mathbb{L a t}_{r}^{n}(K)$. We take the special case $\mathfrak{R}=\mathfrak{W}$, the scheme of Witt vectors over $k$. It is possible to work with the infinite dimensional scheme $p^{-r} F$ and a fixed lattice of codimension $n r$ in it, $L \subseteq p^{-r} F$. Write $M=p^{-r} F$. Let $M$ have basis $e_{i}^{\prime}=p^{-r} e_{i}$ and let $u_{1}, \ldots, u_{n}$ be a basis for $L$. An infinitesimal lattice in $M^{\varepsilon}$ is just a free $\mathcal{O}^{\varepsilon}$-submodule of it of the appropriate $k[\varepsilon]$-co-rank. In particular the $\mathcal{O}^{\varepsilon}$-lattices in $M^{\varepsilon}$ lying over $L$ are just the free $\mathcal{O}^{\varepsilon}$-submodules of $M^{\varepsilon}$ which reduce to $L$. These $\mathcal{O}^{\varepsilon}$-modules are exactly the tangent space to $L$ in $\mathbb{L}_{\text {at }}{ }_{r}(K)$.

LEMMA 18 . Let $\tilde{L}$ be an element of the tangent space to $L$ in $\mathbb{L a t}_{r}^{n}(K)$. Then

(1) $D_{\mathcal{O}} \tilde{L}=\left\{(0, \gamma): \gamma \in D_{L}\right\}$.

(2) $\{\gamma:(0, \gamma) \in \tilde{L}\}=D_{L}$.

Proof. The $\mathcal{O}^{\varepsilon}$-lattice, $\tilde{L}$, admits a basis $\left\{\tilde{u}_{1}, \tilde{u}_{2}, \ldots, \tilde{u}_{n}\right\}, \tilde{u}_{i} \in M^{\varepsilon}$, which reduces to the $L$-basis $\left\{u_{1}, \ldots, u_{n}\right\}$ Consequently, $L=\bigsqcup_{i} \mathcal{O} u_{i}$. It follows that $D_{L}=\bigsqcup_{i} D_{\mathcal{O}} u_{i}$.

Since $\tilde{u}_{i}$ reduces to $u_{i}$, it follows that $\tilde{u}_{i}=\left(u_{i}, \lambda_{u_{i}}^{(i)}\right)$. Hence by Proposition $15, D_{\mathcal{O}} \tilde{L}=$ $\coprod_{i} D_{\mathcal{O}}\left(u_{i}, \lambda_{u_{i}}^{(i)}\right)=\coprod_{i}\left(0, D_{\mathcal{O}} u_{i}\right)$. This proves (1).

Suppose $(0, \gamma) \in \tilde{L}$. Freeness implies that $(0, \gamma)=\sum_{1}^{n}\left(x_{i}, \delta_{x_{i}}^{(i)}\right)\left(u_{i}, \lambda_{u_{i}}^{(i)}\right)=$ $\left(\sum_{1}^{n} x_{i} u_{i}, \sum_{1}^{n}\left[\left(x_{i} \lambda^{(i)}\right)_{x_{i} u_{i}}+\left(\delta^{(i)} u_{i}\right)_{x_{i} u_{i}}\right]\right)$. But this means that $\sum_{1}^{n} x_{i} u_{i}=0$. Since the $u_{i}$ are a basis this means that the $x_{i}$ are all 0 . But then the sum reduces to $\left(0, \sum_{1}^{n} \delta^{(i)} u_{i}\right)$. By (1), the sum is a typical element of $D_{L}$. 
LEMMA 19. Let $\tilde{L}$ be an element of the Zariski tangent space to $\mathbb{L a t}_{r}^{n}(K)$ at L. Then for each $u \in L$, there is an element, $\left(u, \lambda_{u}\right)$ in $\tilde{L}$. Let $\phi(u)$ be the class of $\lambda_{u}$ in $D_{M} / D_{L}$. Then the following hold.

(1) $\phi$ is an $\mathcal{O}$-linear map from $L$ to $D_{M} / D_{L}$.

(2) $p^{(n-1) r} F \subseteq L$ and $\phi\left(p^{(n-1) r} F\right)=(0)$.

(3) If $\phi \in \operatorname{Hom}_{\mathcal{O}}\left(L, D_{M} / D_{L}\right)$ and $\phi\left(p^{(n-1) r} F\right)=(0)$ then the $\mathcal{O}^{\varepsilon}$-module generated by all elements $\left(u, \lambda_{u}\right)$ such that $\lambda \equiv \phi(u) \bmod D_{L}$ is a $\mathcal{O}^{\varepsilon}$-lattice reducing to $L$ and the map associated to it by the process above is just $\phi$.

Proof. Write $D_{L}$ for the infinitesimal module of $L$. Now $\tilde{L} \subseteq M^{\varepsilon}$ and the image of $\tilde{L}$ in $M$ is $L$. Hence for any $u \in L$ there is an element $\tilde{u} \in \tilde{L}$ which maps to it in $L$. Hence $\tilde{u}=\left(u, \lambda_{u}\right)$ for some $\lambda \in D_{M}$. Let $\phi(u)$ be the class of $\lambda$ in $D_{M} / D_{L}$. If $\tilde{u}^{\prime}=$ $\left(u, \gamma_{u}\right)$ is another element of $\tilde{L}$ lying above $u$, then $(0, \lambda-\gamma)$ is in $\tilde{L}$ and so by Lemma 18 , $\lambda-\gamma \in D_{L}$. Consequently, the class of $\lambda \bmod D_{L}$ is well defined. Let $\phi(u)$ be this class. If $x \in \mathcal{O}$ and $\left(v, \gamma_{v}\right) \in \tilde{L}$, notice that $(x, 0)\left(u, \lambda_{u}\right)+\left(v, \gamma_{v}\right) \in \tilde{L}$. This expression is equal to $\left(x u+v, x \lambda_{x u+v}+\gamma_{x u+v}\right)$. Since this element is in $\tilde{L}$ it follows that $\phi(x u+v)$ is the class of $x \lambda+\gamma$. This proves linearity.

Now $p^{(n-1) r} F \subseteq L$ because that is so for every lattice in $\mathbb{L a t}_{r}^{n}(K)$. Consequently, $p^{(n-1) r} M^{\varepsilon} \subseteq \tilde{L}$. This means that for any $m \in F,\left(p^{(n-1) r} m, 0\right) \in \tilde{L}$. By the definition of $\phi$, (2) follows.

Converseley, given $\phi$, let $u_{1}, \ldots, u_{n}$ be a basis for $L$. Choose $\gamma_{i}$ so that $\gamma_{i} \equiv \phi\left(u_{i}\right)$ $\bmod D_{L}$. It is clear that the elements, $\left(u_{i},\left(\gamma_{i}\right)_{u_{i}}\right)$, are a basis for a $\mathcal{O}^{\varepsilon}$-lattice which we will call $\tilde{L}$. It is also clear that the map constructed from $\tilde{L}$ by the procedure above is $\phi$.

Now $M_{I}$, the invariant co-normal vectors are dual to the invariant normal vectors which we denote $M_{I}^{*}$. Recall that the linear maps from $M_{I}$ to the additive characters of $L$ are exactly the space of co-additive co-normal vectors to $L$. This space can be written, $M_{I}^{*} \otimes \mathcal{A}(L)$ where $\mathcal{A}(L)$ is the group of additive characters of $L$.

THEOREM 6. Let $L$ be a point in $\mathbb{L a t}_{r}^{n}(K)$ and let $I$ be the ideal defining it in $k\left[F_{r}\right]$. Let $M_{I}=I /\left(I \cap \mathfrak{m}_{F_{r}}^{2}\right)$. Then $M_{I}^{*}$ is canonically an $\mathcal{O}$-module. The tangent space to $\mathbb{L a t}_{r}^{n}(K)$ at $L$ is $\operatorname{Hom}_{\mathcal{O}}\left(L /\left(p L+p^{(n-1) r} F\right), M_{I}^{*}\right)$. When $L$ is of the form $L_{1}+p^{(n-1) r} F$, for $L_{1} a$ direct summand of $p^{-r} F$ of rank $n-1$, it is of dimension $n(n-1) r$. In all other cases it is of dimension $n^{2} r$. The points where it is of minimal dimension are exactly the $S L(n, \mathcal{O})$ orbit of any lattice of type $(-r, \ldots,-r,(n-1) r)$; they are exactly the smooth locus and they constitute an open set with complement of codimension 2.

Proof. It is clear that $M_{I}^{*}=D_{M} / D_{L}$ and so Lemma 19 establishes a bijective correspondence between the tangent space at $L$ and $\operatorname{Hom}_{\mathcal{O}}\left(L /\left(p L+p^{(n-1) r} F\right), M_{I}^{*}\right)$. It is clear that if $L$ is not of type $(-r, \ldots,-r,(n-1) r)$, then $p L \supseteq p^{(n-1) r} F$ and so $L /\left(p L+p^{(n-1) r} F\right)$ is of dimension $n$. When it is of type $(-r, \ldots,-r,(n-1) r)$, then $p L \nsupseteq p^{(n-1) r} F$ and this quotient is of dimension $n-1$. Thus the Hom in question is of dimension $n^{2} r$ in the first case and of dimension $n(n-1) r$ in the second. The homogeneity statement follows from 
Proposition 10. The smoothness statement is an application of the classical Zariski criterion of simplicity.

3.6. Some concluding thoughts on the general case: This paper has been for the most part concerned with spaces of special lattices. In this section we wish to indicate how to deal with general homogeneous spaces of the form $G / M$, where $G=G(K)$ is the group of $K$ points of a split semisimple group and $M$ is a bounded subgroup in the sense of Bruhat Tits. Here $K$ is taken to be either the field of Laurent series over an algebraically closed field or the Hilbert class field. More properly, $K$ is the fraction field of $W(k)$, where $W$ is a $w$-scheme (see Hypotheses 1 in Subsection 2.6). Write $\pi$ for the uniformizing parameter of $\mathcal{O}$. We work in this section with complete perfect topological schemes. Although further extensions of these remarks and results are straightforward we reserve a survey of the possibilities for a future work.

DEFINITION 26. Let $\mathcal{O}$ be a complete discrete valuation ring with fraction field, $K$, and algebraically closed residue class field, $k$. Let $R \subseteq \mathcal{O}$ be a base ring and let $G$ be a split semisimple group over $K$ defined over $R$. A subgroup, $P$, of $G(K)$ will be called a BT-subgroup of $G(K)$ if there is a group scheme $\tilde{M}$ over $\mathcal{O}$ such that:

(1) $\tilde{M}$ is flat and of finite type over $\mathcal{O}$.

(2) $\tilde{M}_{K} \simeq G$.

(3) $\quad P=\tilde{M}(\mathcal{O})$.

When $P=\tilde{M}(\mathcal{O})$, an $\mathcal{O}$-group scheme $\tilde{M}$ satisfying these conditions will be called a Bruhat Tits group scheme associated to $P$.

In [BT2], Bruhat and Tits demonstrated one of their more celebrated results. The group $G(K)$ is the group associated to a Tits system with Weyl group equal to the affine Weyl group of $G(K)$ which we will denote $\tilde{W}$. The Tits system is the one for which $B$ is an Iwahori subgroup and $N$ is the normalizer of a maximal $K$-torus. A subset is called bounded if it is contained in a finite union of double cosets, $B w B$, where $w \in \tilde{W}$ and $B$ is the Iwahori subgroup. The result alluded to is that every bounded subgroup of $G(K)$, sufficiently large in a sense precisely defined there, is the group of $\mathcal{O}$-points of some Bruhat Tits group scheme with generic fiber, $G_{K}$. The closures, $\overline{B w B}$ are affine schemes (Pro-schemes, but these are only schemes not of finite type over $k$.). The following only recapitulates arguments in [BT2]. We include it for illustration.

Proposition 16. Let $G=G(K)$ and let $M \subseteq G$ be a BT-subgroup of $G$ with associated Bruhat Tits scheme $\tilde{M}$. Then there is a finite dimensional $K$-representation of $G, P$, and a maximal lattice, $L \subseteq P$ so that $M$ is the pointwise stabilizer of $L$ in $P$.

Proof. Let $\tilde{M}=\operatorname{Spec} C$ where $C$ is a finitely generated $\mathcal{O}$-algebra. Then $G=$ $\operatorname{Spec}(K[G]), C \otimes \mathcal{O} K=K[G]$. Let $\mu: C \rightarrow C \otimes C$ be the co-multiplication. By an elementary argument (see [Sp, p.29, 2.3.6]), there is an $\mathcal{O}$-finite right $M$-translation invariant $\mathcal{O}$-submodule, $N_{0} \subseteq C$ such that $N_{0}$ generates $C$ as an $\mathcal{O}$-algebra. We may assume that $N_{0}$ contains the unit element and that it contains a set of generators for the ideal defining the 
identity element in $\tilde{M}$. Right translation invariance holds if and only if $\mu\left(N_{0}\right) \subseteq N_{0} \otimes_{\mathcal{O}} C$. Let $N=\left\{n: n \in C\right.$, there exists $a \in \mathcal{O}$ such that $\left.a n \in N_{0}\right\}$.

Consider $N$. Then since $N \supseteq N_{0}$, it certainly generates $C$. Since $\mathcal{O}$ is a discrete valuation ring we may choose a basis for $N$ and extend it to a linear basis of $C$. Since $C$ is of finite type we may assume that the basis is countable. Let $n_{1}, \ldots, n_{q}$ be the basis of $N$ and let $n_{q+1}, \ldots$ denote the extension of this basis to $C$. Then $\left\{n_{i} \otimes 1\right\}, i \leq q$ is a basis for $N \otimes C$ over $C$ and $\left\{n_{i} \otimes 1\right\}$ is a $1 \otimes C$ basis of $C \otimes C$. Let $\mu(x)=\sum_{i} n_{i} \otimes b_{i}$. Then $\mu(a x)=\sum_{i} n_{i} \otimes a b_{i}$. Since $a x \in N$ then $\mu(a x) \in N \otimes C$. Hence $a b_{i}=0$ for all $i \geq q$. Thus $b_{i}=0$ for all $i \geq q$ and $b_{i} \in C$ otherwise. That is, $N$ is right translation invariant also.

Let $P=N \otimes_{\mathcal{O}} K$. Then since $N$ generates $C$ and $G$ is the generic fiber of $\tilde{M}, P$ generates $K[G]$ and since $\mu(N) \subseteq N \otimes C$ it follows that $\mu(P) \subseteq P \otimes K[G]$. Since $P$ generates $K[G]$ and is right translation invariant, the right translation representation of $G$ on $P$ is faithful. Suppose that $g \in G$ stabilizes $N$, that is, that $g N \subseteq N$. For $i \leq q$ let $\mu\left(n_{i}\right)=\sum_{j=1}^{q} n_{j} \otimes a_{j . i}$. Then $g n_{i}=\sum_{j=1}^{q} a_{j, i}(g) n_{j}$. Then $g n_{i} \in N$ if and only if $a_{j, i}(g) \in \mathcal{O}$ for all $i, j \leq q$.

Consider the $a_{j, i}$. Apply the homomorphism, evaluation at the identity, to the left hand side of the equation, $\mu\left(n_{i}\right)=\sum_{j=1}^{q} n_{j} \otimes a_{j, i}$. We obtain the equation, $n_{i}=\sum_{i=1}^{q} n_{j} a_{j, i}$. This means that the $a_{j, i}$ generate $C$. Since this is so and since $a_{j, i}(g) \in \mathcal{O}$ it follows that $g \in \tilde{M}(\mathcal{O})$. That is, if $g N=N, g \in M$. The converse inclusion is clear.

Corollary 5. Let $G=G(K)$ and let $P$ be a closed subgroup. Then the following are equivalent.

(1) $P$ is closed and bounded.

(2) $P$ is a $k$-closed subscheme of a BT-subgroup of $G$.

(3) $P$ is a quasicompact subgroup of $G$.

Proof. Item (2) implies (1) and (1) implies (3), and so all that must be shown is that (3) implies (2). To see this choose a faithful representation of $G$ in the category of $K$-groups, $\rho: G \rightarrow G L(n, K)$. In the category of perfect complete topological schemes this gives rise to an action $G \times K^{n} \rightarrow K^{n}$. Choose a lattice $N \subseteq K^{n}$. Then $P \times N$ is quasi-compact and so its image in $K^{n}$ under the action, which we denote $P N$, is quasi-compact. The sets $\pi^{r} N$ are an expanding open cover of $K^{n}$ and so $P N \subseteq \pi^{s} N$ for some $s$. This means that $P N$ spans some lattice $L$ contained in $\pi^{s} N$. Thus $P \subseteq G L(L)$ which is a BT-subgroup scheme. Finally $G L(L) \cap G$ is a BT-subgroup of $G$.

This brings us to our concluding observation. If $G=G(K)$ and $P \subseteq G$ is a parahoric subgroup, then by the theorem of Bruhat and Tits there is a representation of $G$ on $K^{n}$ so that $P$ is the stabilizer of a lattice. Hence in the category of complete perfect schemes $G / P$ can be represented as an orbit in $\mathbb{L}^{n}(K)$. In the sequel to this work we will address the question of whether this homogeneous space has a canonical description in the category of ordinary topological schemes. 


\section{REFERENCES}

[AT] E. AbE AND M. TAKEUCHI, Groups associated with some types of infinite dimensional Lie algebras J. Algebra 146 (1992), 385-404.

[ADK] E. ARbarello, C. De Concini And V. KaC, The infinite wedge representation and the reciprocity law for algebraic curves, Theta functions -Bowdoin, 1987, Part I (Brunswick, ME,1987), 171-190, Proc. Sympos. Pure Math. 49 Amer. Math. Soc., Providence, RI, 1989.

[IB1] I. BARSotti, Moduli canonici é gruppi analitici commutative, Ann. Scuola Norm. Sup. Pisa, 13 (1959), 303-372.

[IB2] I. BARSOTTI, Analytical methods for Abelian varieties in positive characteristic, Colloq. Théorie Groupes Algébriques, C. B. R. M., Bruxelles, 1962, 77-85, Gauthier-Villars, Paris 1962.

[IB3] I. BARsott, Metodi analitici per varieta Abeliane in caratteristica positiva, Chaps. I and II, Ann. Scuola Norm. Sup. Pisa, 18 (1964) 1-25; Chaps. III, IV, V: ibid. 19 (1965), 277-330; Chaps. VI, VII: ibid. 20 (1966), 101-137, 331-365.

[BL] A. Beauville And Y. Laszlo, Conformal blocks and generalized theta functions, Comm. Math. Phys. 164 (1994), 385-419.

[BLS] A. Beauville, Y. Laszlo and C. Sorger, The Picard group of the moduli of $G$-bundles on a curve, Compositio Math. 112, (1998), 183-216.

[Bo] N. Bourbaki, Groupes et algébres de Lie, Chapitres 4, 5, et 6, Éléments de mathématiques Fascicule XXXIV Hermann, Paris, 1968.

[BT1] F. BRUHAT AND J. TITs, Groupes réductifs sur un corps local, I, Publ. Math. Inst. Hautes Études Sci. 41 (1972), 5-251.

[BT2] F. BRuhat And J. Tits, Groups réductifs sur un corps local, II, Publ. Math. Inst. Hautes Études Sci. 60 (1984), 5-184.

[SGAD] M. Demazure And A. Grothendieck with M. Artin, J. E. Bertin, P. Gabriel, M. Raynaud and J.-P. Serre, Schémas en Groupes, I, séminaire de géomètrie algebrique du Bois Marie 1962/64, Lecture Notes in Mathematics 151, Springer Verlag, Berlin, Heidelberg, New York, 1970, II 152, III Ibid. 1531970.

[F] G. Faltings, A proof for the Verlinde formula J. Algebraic Geom. 3 (1994), 347-374.

[Ga] H. Garland, The arithmetic theory of loop groups, Publ. Math. Inst. Hautes Études Sci. 52 (1980), 5-136.

[MJG1] M. J. GreenBerg, Schemata over local rings, Ann. Math. 73 (1961), 624-648.

[MJG2] M. J. Greenberg, Schemata over local rings II, Ann. Math. 78 (1963), 256-266.

[EGA] A. Grothendieck And J. Dieudonné, Eléments de géomètrie algébrique, I, Grundlehren der Math. Wissenschaften 166 Springer Verlag, New York, Heidelberg, Berlin, 1971, II, Publ. Math. Inst. Hautes Études Sci. 8 (1961), III, Ibid. 11(1961) and 17(1963), IV, Ibid. 20 (1964).

[Ha] R. Hartshorne, Algebraic Geometry, Springer Verlag, New York, Heidelberg, Berlin, 1977.

[IM] N. IWAhori AND H. Matsumoto, On some Bruhat decomposition and the structure of the Hecke ring of $p$-adic Chevalley groups, Publ. Math. Inst. Hautes Études Sci. 25 (1965), 5-48.

[K] V. G. KAC, Constructing groups associated to infinite dimensional Lie algebras, Infinite dimensional groups with applications (V. Kac, ed.), Mathematical Sciences Research Institute Publications, vol. 4 , Springer Verlag, New York, Berlin, Heidelberg, Paris, Tokyo, 1985.

[KP] V. G. KaC And D. Peterson, Regular functions on some infinite dimensional groups, Arithmetic and geometry (M. Artin and J. Tate, eds.), 141-166, Progr. Math. 36, Birkhäuser, Boston, Basel, Berlin, 1983.

[KNR] S. KUMAR, M. S. NARASIMHAN AND A. RAMANATHAN, Infinite Grassmannians and moduli spaces of $G$-bundles, Math. Ann. 300 (1994), 41-75.

[Ma] O. Mathieu, Formules de caractéres pour les algébres de Kac-Moody génèrales, Astérisque 159-160 (1988), 269 pp. 
[Mt] H. Matsumoto, Sur les sous-groupes arithmétiques des groupes semi-simples déployés, Ann. Sci. École Norm. Sup. (4)2 (1969), 1-62.

[Mo] C. C. Moore, Group extensions of $p$-adic and adelic linear groups, Publ. Math. Inst. Hautes Études Sci. 35 (1968), 5-70.

[Mu D. Mumford, Lectures on curves on an algebraic surface with a section by G. M. Bergman, Princeton University Press, Princeton, N.J., 1966.

[PS] A. Pressley And G. Segal, Loop Groups, Oxford Science Publications, Clarendon Press, Oxford, 1986.

[S1] J.-P. SERRE, Corps locaux, Publications de l'institut de mathématiques de l'université de Nancago VIII, Hermann, Paris, 1962.

[S2] J.-P. SERRE, Groupes proalgébriques, Publ. Math. Inst. Hautes Études Sci. 7 (1960), 5-67.

[Sh] I. N. ShafAREVICH, On some infinite dimensional groups II, Izv. Akad Nauk SSSR Ser. Mat. 45 (1981), 216-226.

[So] C. Sorger, Lectures on moduli of Principal $G$-bundles over algebraic curves, School on Algebraic Geometry (Trieste, 1999), 1-57, ICTP Lect. Notes, 1, Abdus Salam Int. Cen. Theoret. Phys. Trieste, 2000.

[Sp] T. A. Springer, Linear Algebraic Groups, Second Edition, Progr. Math. 9, Birkhäuser, Boston, Basel, Berlin, 1998.

[St] R. Steinberg, Générateurs, relations et revêtements de groupes algébriques, Colloq. Théorie Groupes Algébriques, C. B. R. M., Bruxelles, 1962, 113-127, Gauthier-Villars, Paris 1962.

[MT] M. TAKEUCHI, Topological coalgebras, J. Algebra 97 (1985), 505-539.

[T1] J. TITS, Uniqueness and presentation of Kac-Moody groups over fields, J. Algegra 105 (1987), 542-573.

[T2] J. TITs, Groupes associés aux algèbres de Kac-Moody, séminaire Bourbaki 41 ème année, 1988-89, Astérisque 177-178 (1989), Exp. No. 700, 7-31.

DEPARTMENT OF MATHEMATICS

THE UNIVERSITY OF ILLINOIS

273 Altgeld HALL

1409 W. GREEN ST.

URBANA IL 61801

U.S.A.

E-mail address: haboush@math.uiuc.edu 